\title{
Redox Non-Innocent Monoatomic Silicon(0) Complex ('Silylone'): Its One-Electron-Reduction Induces an Intramolecular One-Electron-Oxidation of Silicon(0) to Silicon(I)
}

Shenglai Yao, Arseni Kostenko, Yun Xiong, Ales Ruzicka, and Matthias Driess*

Table of Content

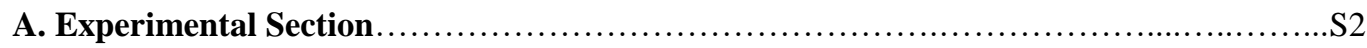

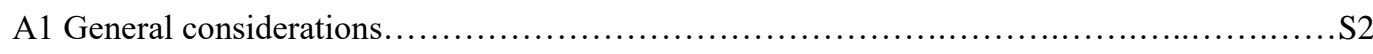

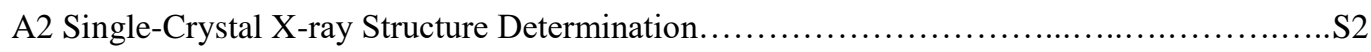

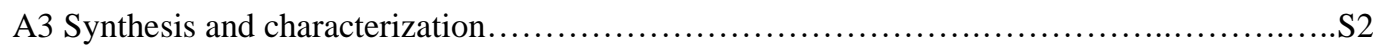

A4 Details of the single crystal X-ray diffraction analyses................................... 8

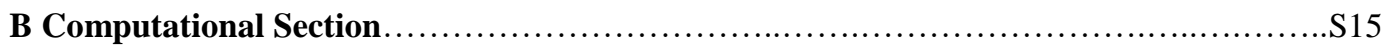

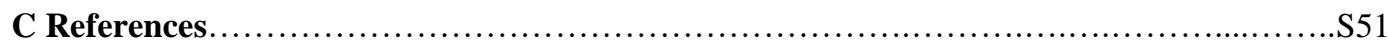




\section{A. Experimental Section}

\section{A1. General Considerations}

All experiments were carried out under dry oxygen-free nitrogen using standard Schlenk techniques or an MBraun glove box fitted with a gas purification and recirculation unit. Solvents were dried by standard methods and freshly distilled prior to use. The starting material, 1,2-bis(N-heterocyclic silylenyl) 1,2dicarba-closo-dedocaborane(12) scaffold, [1,2-(LSi) $\left.)_{2} \mathrm{C}_{2} \mathrm{~B}_{10} \mathrm{H}_{10} ; \quad \mathrm{L}=\mathrm{PhC}(\mathrm{N} t \mathrm{Bu})_{2}\right] \mathbf{1}^{1}$ and $\mathrm{NHC}-\mathrm{SiCl}_{2}$ $(\mathrm{NHC}=1,3 \text {-bis(2,6-diisopropylphenyl)imidazol-2-ylidene })^{2}$ 2were prepared according to the literature procedures. Potassium graphite was prepared by reacting potassium with previously dried graphite in a 1:8 ratio at $160{ }^{\circ} \mathrm{C}$ for $2 \mathrm{~h}$ under dried nitrogen. The NMR spectra were recorded with Bruker spectrometers ARX200 and AV400 referenced to residual solvent signals as internal standards. Abbreviations: $s=\operatorname{singlet;} d=$ doublet; $t=$ triplet; sept $=$ septet; $m=$ multiplet; $b r=$ broad. Elemental analyses were performed by the analytical labor service in the Institute of Chemistry, Technical University of Berlin, Germany. High-resolution ESI-MS were measured on a Thermo Scientific LTQ orbitrap XL. IR spectra were measured with a Nicolet iS5 FT-IR-Spectrometer from the company of Thermo Scientific.

\section{A2. Single-Crystal X-ray Structure Determination}

The crystal was mounted on a glass capillary in per-fluorinated oil and measured in a cold $\mathrm{N}_{2}$ flow. The data of $\mathbf{3}$ and $\mathbf{4}$ were collected on an Oxford Diffraction Supernova, Single source at offset, Atlas at 150 $\mathrm{K}\left(\mathrm{Cu}\right.$ - K $\alpha$-radiation, $\lambda=1.5418 \AA$ ). The structure was solved by direct method and refined on $F^{2}$ with the SHELX $-97^{3}$ software package. The positions of the $\mathrm{H}$ atoms were calculated and considered isotropically according to a riding model. In the molecular structure of compound $\mathbf{4}$, the carborane moiety is disordered over two orientations with an occupancy ratio of 0.50:0.50. One of the tert-butyl groups is disordered over two orientations with an occupancy ratio of 0.53:0.47. In addition, there is a free but highly disordered THF molecule within the asymmetric unit which was treated using the SQUEEZE routine in PLATON.

\section{A3. Synthesis and Characterization}

\section{Synthesis of 2}

To a Schlenk tube charged with $1(0.22 \mathrm{~g}, 0.33 \mathrm{mmol})$ and potassium graphite $(0.090 \mathrm{~g}, 0.73 \mathrm{mmol})$ was added $15 \mathrm{~mL}$ THF at room temperature with stirring. After stirring at room temperature for $1.5 \mathrm{~h}$, the resulting suspension was filtered. The orange solution was concentrated under reduced pressure and cooled at $-30^{\circ} \mathrm{C}$ overnight to give compound 2 as a yellow solid (Yield: $0.29 \mathrm{~g}, 92 \%$ ). Single crystals of 2 with insufficient quality were obtained from recrystallization of $\mathbf{2}$ in THF at room temperature. M.p. $247{ }^{\circ} \mathrm{C}$ (decomp.). ${ }^{1} \mathbf{H}$ NMR (400 MHz, D 8 -THF, 298K): $\delta=1.21$ (s, $\left.36 \mathrm{H}, \mathrm{NC}\left(\mathrm{CH}_{3}\right)_{3}\right), 7.24-7.37$ (m, 8 $\mathrm{H}, \mathrm{Ph}-H), 7.61-7.66 \mathrm{ppm}(\mathrm{m}, 2 \mathrm{H}, \mathrm{Ph}-H) .{ }^{13} \mathbf{C}\left\{{ }^{1} \mathbf{H}\right\}$ NMR (100 MHz, D $\left.-\mathrm{THF}, 298 \mathrm{~K}\right): \delta=33.0(\mathrm{~s}$, $\left.\mathrm{NC}\left(\mathrm{CH}_{3}\right)_{3}\right), 53.1\left(\mathrm{~s}, \mathrm{NC}\left(\mathrm{CH}_{3}\right)_{3}\right), 127.8,128.1,129.4,130.4,132.6,137.4$ (s, Ph- $\left.\mathrm{C}\right), 156.0$ (s, NCN). ${ }^{29} \mathbf{S i}\left\{{ }^{1} \mathbf{H}\right\}$ NMR $\left(99.49 \mathrm{MHz}, \mathrm{D}_{8}-\mathrm{THF}, 298 \mathrm{~K}\right): \delta=57.0 \mathrm{ppm} .{ }^{11} \mathbf{B}\left\{{ }^{1} \mathbf{H}\right\} \mathbf{N M R}\left(64 \mathrm{MHz}, \mathrm{D}_{8}-\mathrm{THF}, 298 \mathrm{~K}\right)$ : $\delta=-26.4,-19.6,-7.9 \mathrm{ppm}(\mathrm{vb})$. Elemental analysis calcd for $\mathrm{C}_{44} \mathrm{H}_{80} \mathrm{~N}_{4} \mathrm{~B}_{10} \mathrm{Si}_{2} \mathrm{~K}_{2} \mathrm{O}_{3}$ : C 55.30, H 8.44, N 5.86; found: C 54.92, H 8.16, N 6.13; IR $\left(\mathrm{cm}^{-1}\right)$ : 2965 (w), 2931 (w), 2901 (w), 2867 (w), 2441 (m, br), 1578 (w), 1473 (w), 1443 (w), 1415 (m), 1389 (w), 1358 (m), 1269(w), 1209 (m), 1159 (w), 1111 (w), 
$1071(w), 1052(w), 1022(w), 974(w), 899(w), 854(w), 796(w), 769(w), 757(w), 705(w), 651(w)$, $613(\mathrm{w})$.

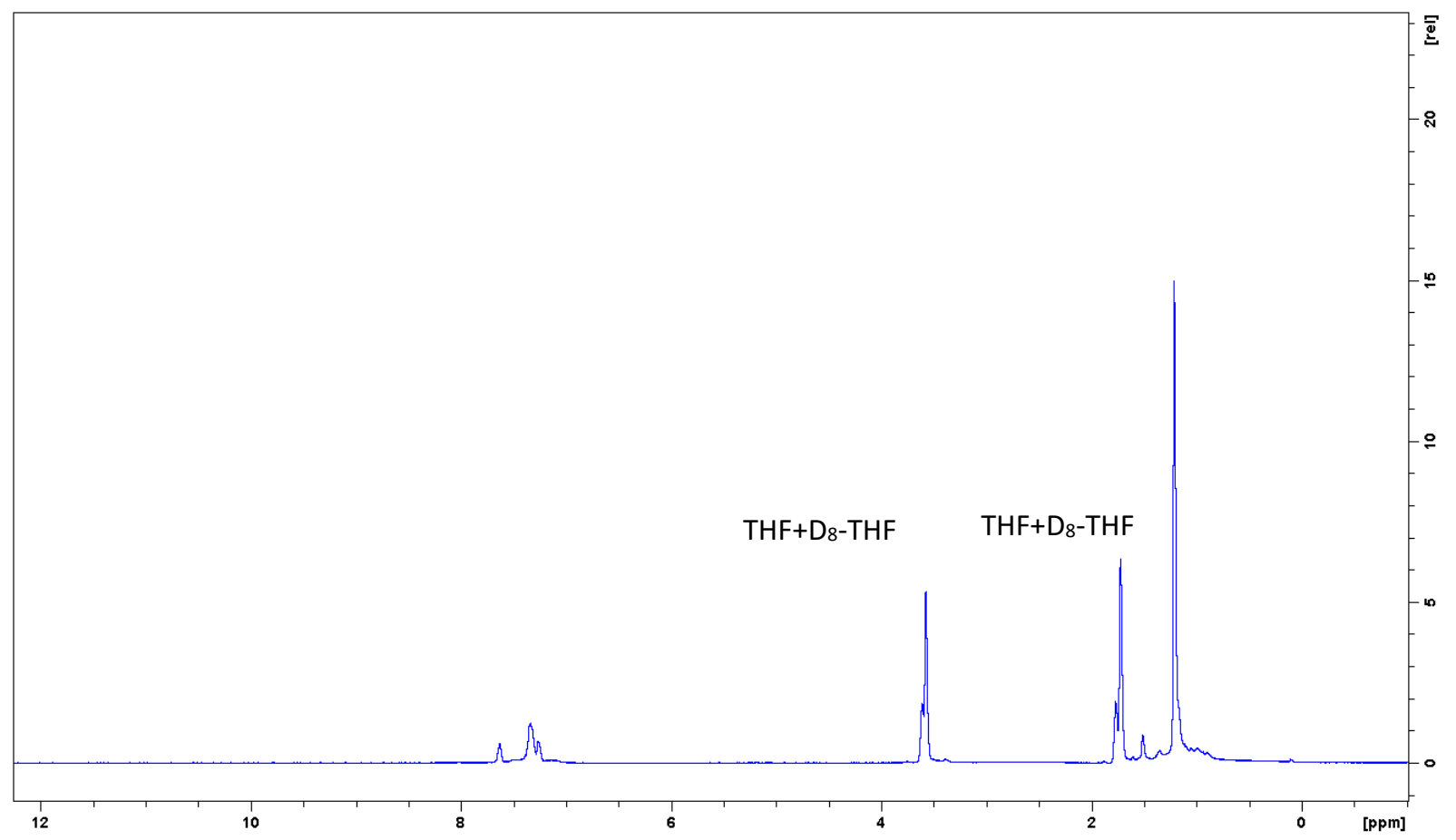

Figure S1. ${ }^{1} \mathrm{H}$ NMR spectrum of compound 2 (400 MHz, D 8 -THF, 298K).

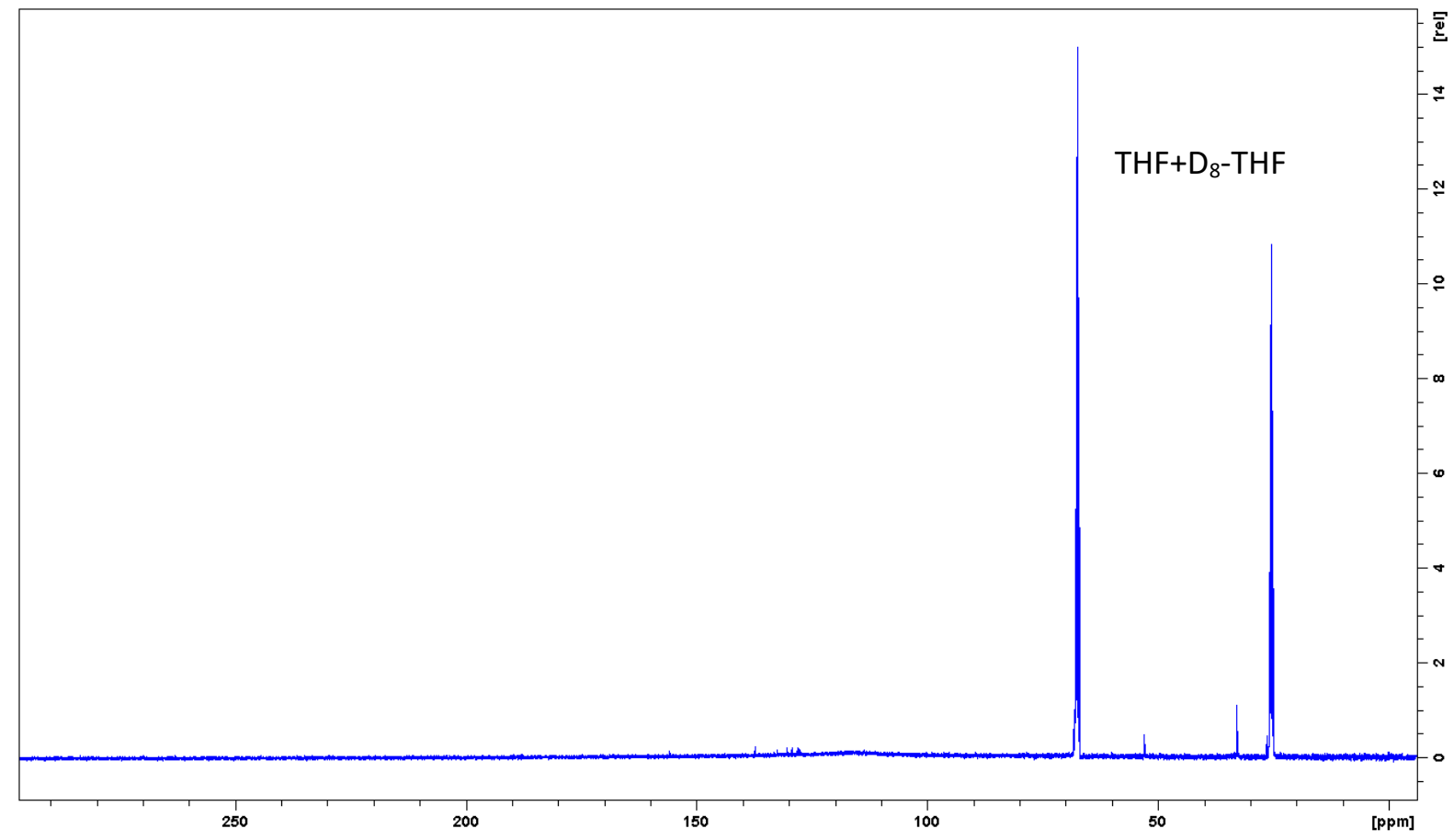

Figure S2. ${ }^{13} \mathrm{C}\left\{{ }^{1} \mathrm{H}\right\}$ NMR spectrum of compound 2 (100 MHz, $\left.\mathrm{D}_{8}-\mathrm{THF}, 298 \mathrm{~K}\right)$. 


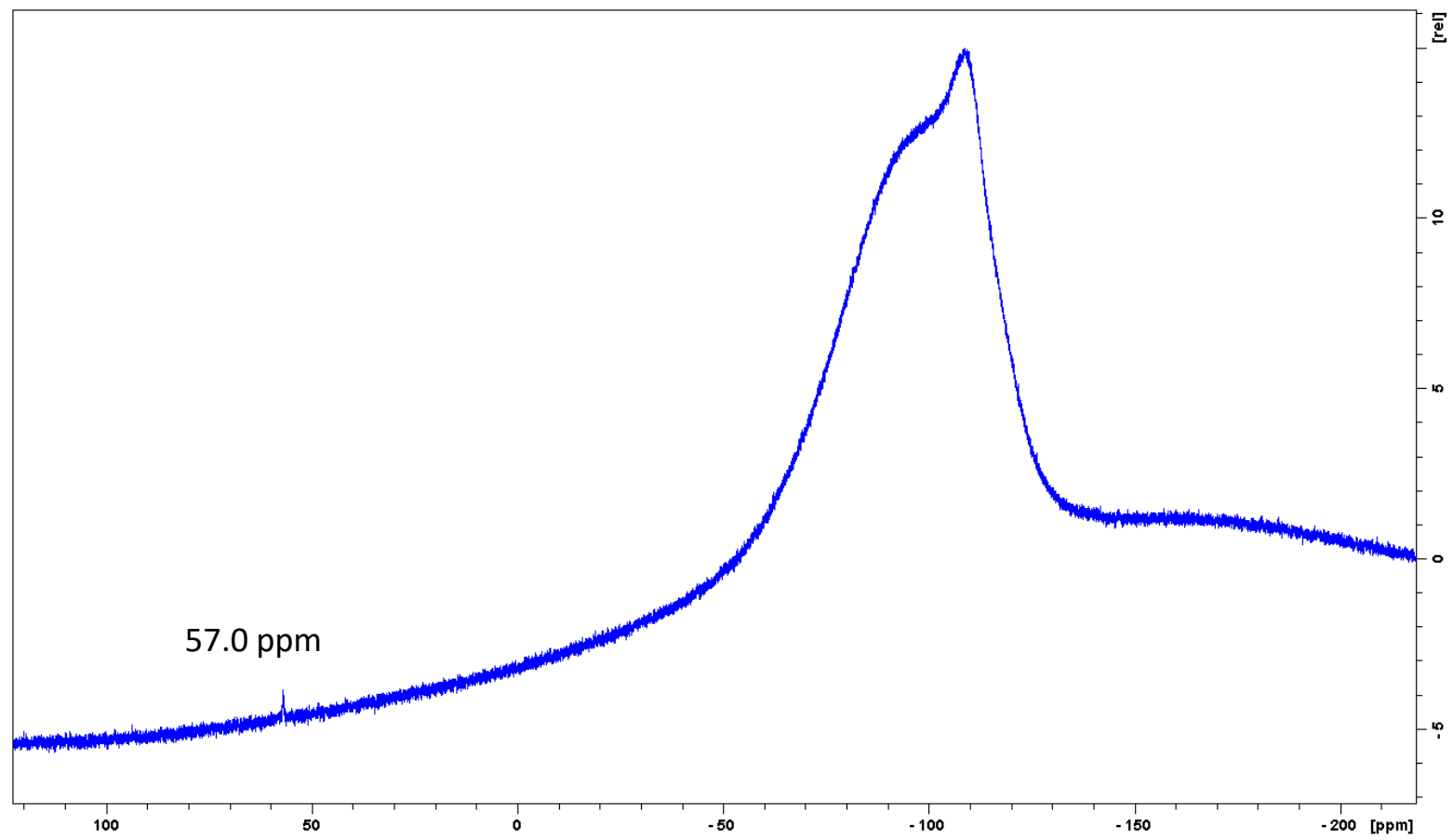

Figure S3. ${ }^{29} \mathrm{Si}\left\{{ }^{1} \mathrm{H}\right\}$ NMR spectrum of compound $2\left(99.49 \mathrm{MHz}, \mathrm{D}_{8}-\mathrm{THF}, 298 \mathrm{~K}\right)$.

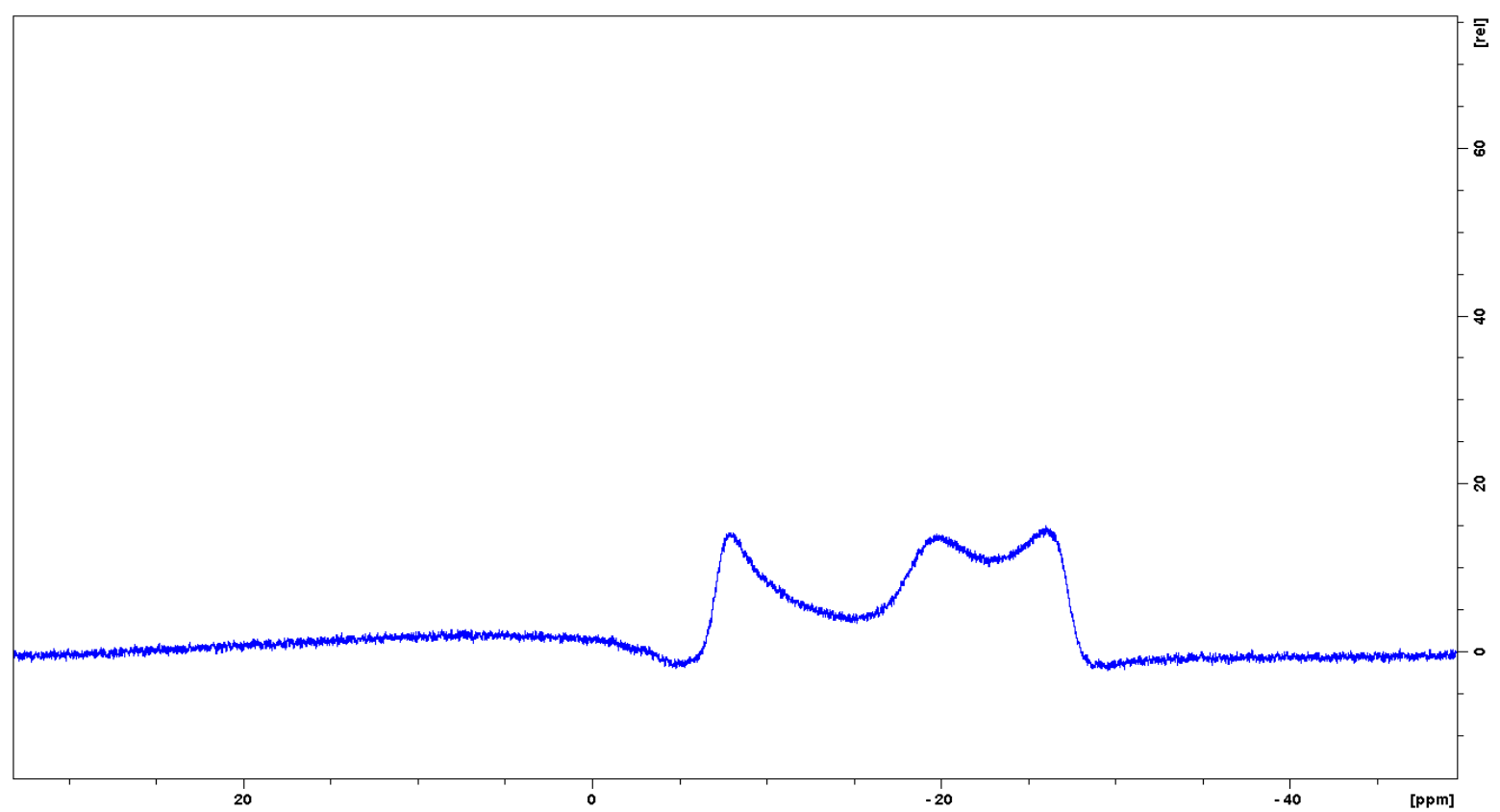

Figure S4. ${ }^{11} \mathrm{~B}\left\{{ }^{1} \mathrm{H}\right\}$ NMR spectrum of compound $2\left(128 \mathrm{MHz}, \mathrm{D}_{8}-\mathrm{THF}, 298 \mathrm{~K}\right)$. 


\section{Synthesis of 3}

To a Schlenk tube charged with $1(0.33 \mathrm{~g}, 0.50 \mathrm{mmol})$ and potassium graphite $(0.14 \mathrm{~g}, 1.04 \mathrm{mmol})$ was added $20 \mathrm{~mL}$ THF at room temperature with stirring. After stirring at room temperature for $1.5 \mathrm{~h}$, the resulting suspension was filtered to give an orange solution. The latter solution was then cooled to $-40{ }^{\circ} \mathrm{C}$ and $\mathrm{NHC}-\mathrm{SiCl}_{2}(0.24 \mathrm{~g}, 0.50 \mathrm{mmol})$ was added with stirring. The reaction mixture was allowed to warm to room temperature and stirred further for $2 \mathrm{~h}$. Volatiles were then removed under vacuum and the residue was extracted with $\mathrm{Et}_{2} \mathrm{O}(2 \times 30 \mathrm{~mL})$. Concentrating and cooling the filtrate at $-30{ }^{\circ} \mathrm{C}$ for $48 \mathrm{~h}$ afforded red crystals of 3 with some yellowish precipitate. Recrystallization with $\mathrm{Et}_{2} \mathrm{O}$ furnished pure compound 3 as red crystals (Yield: 0.21 g, 61 \%). M.p. $297{ }^{\circ} \mathrm{C}$ (decomp.). ${ }^{1} \mathbf{H}$ NMR (400 MHz, D8-THF, 298K): $\delta=1.31$ (s, $\left.36 \mathrm{H}, \mathrm{NC}\left(\mathrm{CH}_{3}\right)_{3}\right), 7.39-7.42(\mathrm{~m}, 2 \mathrm{H}, \mathrm{Ph}-H), 7.48-7.56(\mathrm{~m}, 6 \mathrm{H}, \mathrm{Ph}-H), 7.66-7.69 \mathrm{ppm}$ $(\mathrm{m}, 2 \mathrm{H}, \mathrm{Ph}-H) .{ }^{13} \mathbf{C}\left\{{ }^{1} \mathbf{H}\right\}$ NMR $\left(100 \mathrm{MHz}, \mathrm{D}_{8}\right.$-THF, 298K): $\delta=32.4\left(\mathrm{~s}, \mathrm{NC}\left(\mathrm{CH}_{3}\right)_{3}\right), 56.6\left(\mathrm{~s}, \mathrm{NC}\left(\mathrm{CH}_{3}\right)_{3}\right)$, ), 82.4 (s, carborane- $C$ ), 128.4, 129.0, 129.3, 131.3, 131.4, 133.3 (s, Ph- $C), 177.6$ (s, NCN). ${ }^{29} \mathbf{S i}\left\{{ }^{1} \mathbf{H}\right\}$ NMR (99.49 MHz, D D THF, $298 \mathrm{~K}): \delta=-263.8\left(\mathrm{~s}, \mathrm{Si}_{2} \mathrm{Si}\right), 50.1 \mathrm{ppm}\left(\mathrm{Si}_{2} \mathrm{Si}\right) .{ }^{11} \mathbf{B}\left\{{ }^{1} \mathbf{H}\right\}$ NMR $\left(64 \mathrm{MHz}, \mathrm{D}_{8}-\mathrm{THF}\right.$, $298 \mathrm{~K}): \delta=-14-2$ ppm (m, vb). ESI-MS: m/z: 689.48810 (calc. $689.48887[\mathrm{M}+\mathrm{H}]^{+}$); Elemental analysis calcd for $\mathrm{C}_{32} \mathrm{H}_{56} \mathrm{~N}_{4} \mathrm{~B}_{10} \mathrm{Si}_{3}$ : C 55.77, $\mathrm{H} 8.19, \mathrm{~N} 8.13$; found: $\mathrm{C} 55.29, \mathrm{H} 8.18, \mathrm{~N} 7.94$; IR $\left(\mathrm{cm}^{-1}\right)$ : 2993 (w), 2971 (w), 2931 (w), 2904 (w), 2968 (w), 2571 (m), 2543 (w), 2520 (w), 1578 (w), $1516(w)$, 1473 (w), 1445 (m), 1400 (s), 1394 (s), 1365 (m), 1257 (m), 1226 (w), 1196 (m), 1153 (w), 1083 (m), $1070(\mathrm{~m}), 1033$ (w), $1022(\mathrm{w}), 970(\mathrm{w}), 927$ (w), $879(\mathrm{w}), 842(\mathrm{w}), 787(\mathrm{~m}), 751(\mathrm{~s}), 739(\mathrm{~m}), 732(\mathrm{~m})$, $706(\mathrm{~m}), 676(\mathrm{w}), 628(\mathrm{~s}), 617(\mathrm{~s}), 578(\mathrm{w}), 569(\mathrm{w})$.

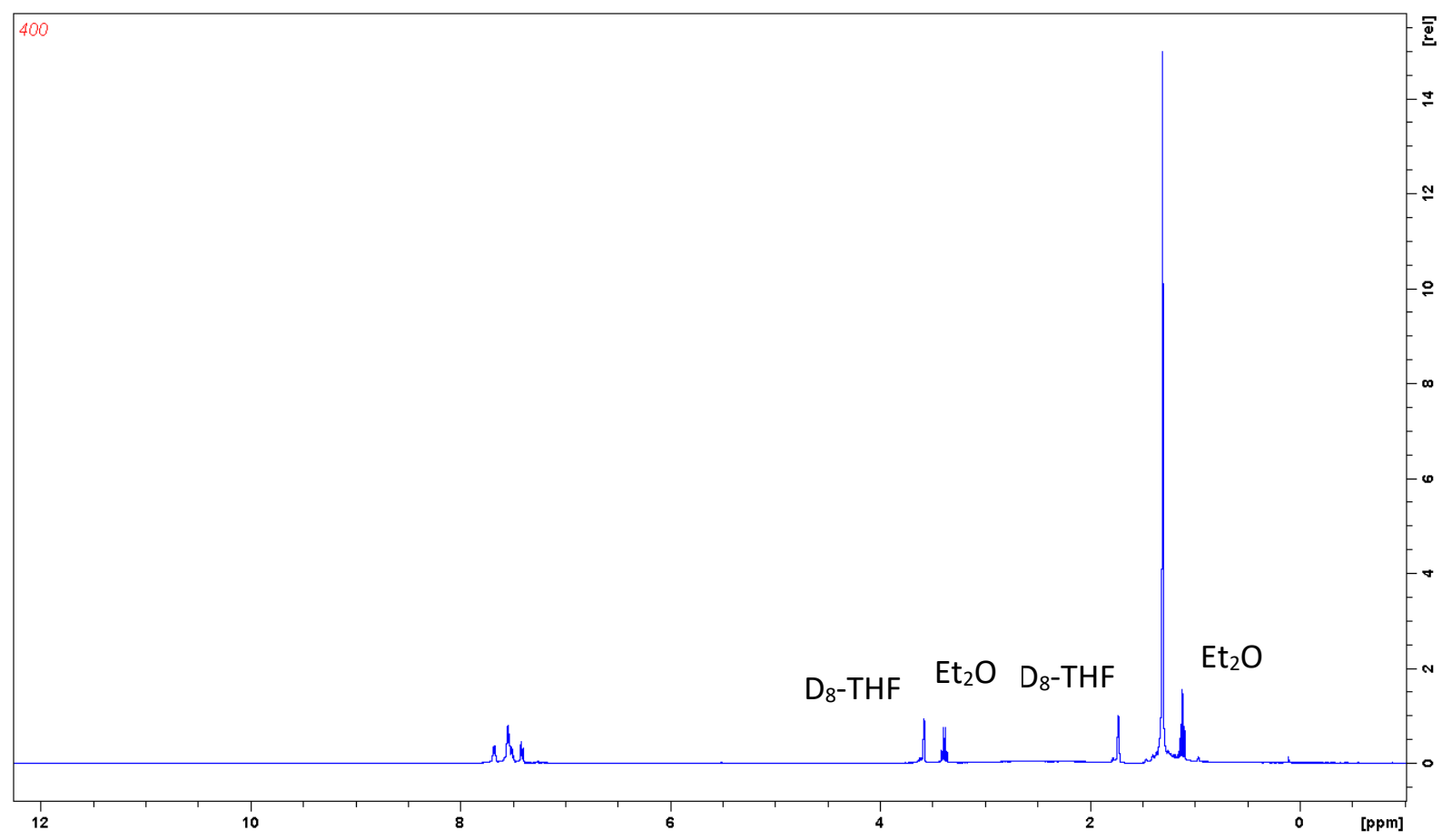

Figure S5. ${ }^{1} \mathrm{H}$ NMR spectrum of compound 3 (400 MHz, D 8 -THF, 298K). 


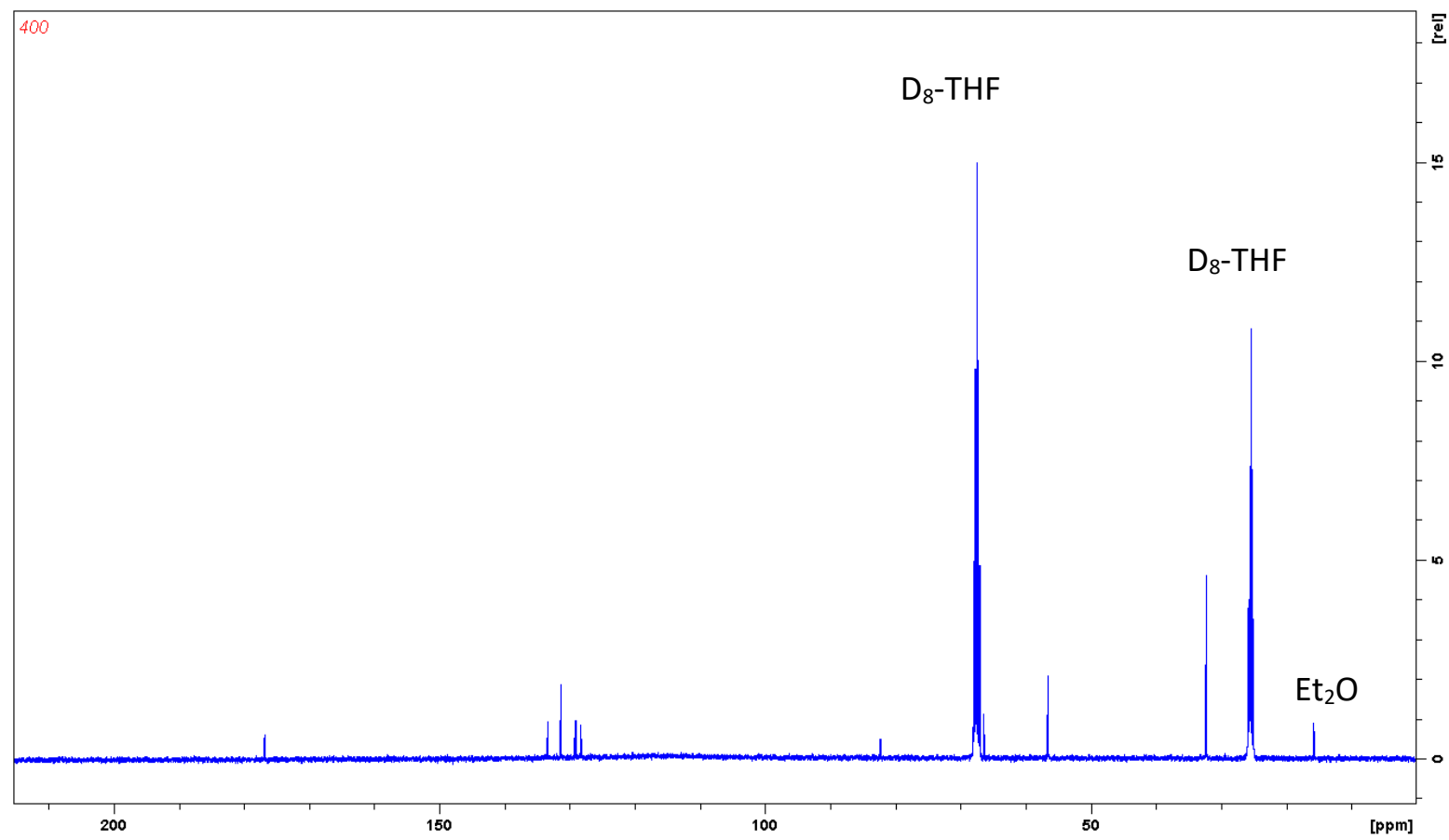

Figure S6. ${ }^{13} \mathrm{C}\left\{{ }^{1} \mathrm{H}\right\}$ NMR spectrum of compound 3 (100 MHz, $\left.\mathrm{D}_{8}-\mathrm{THF}, 298 \mathrm{~K}\right)$.

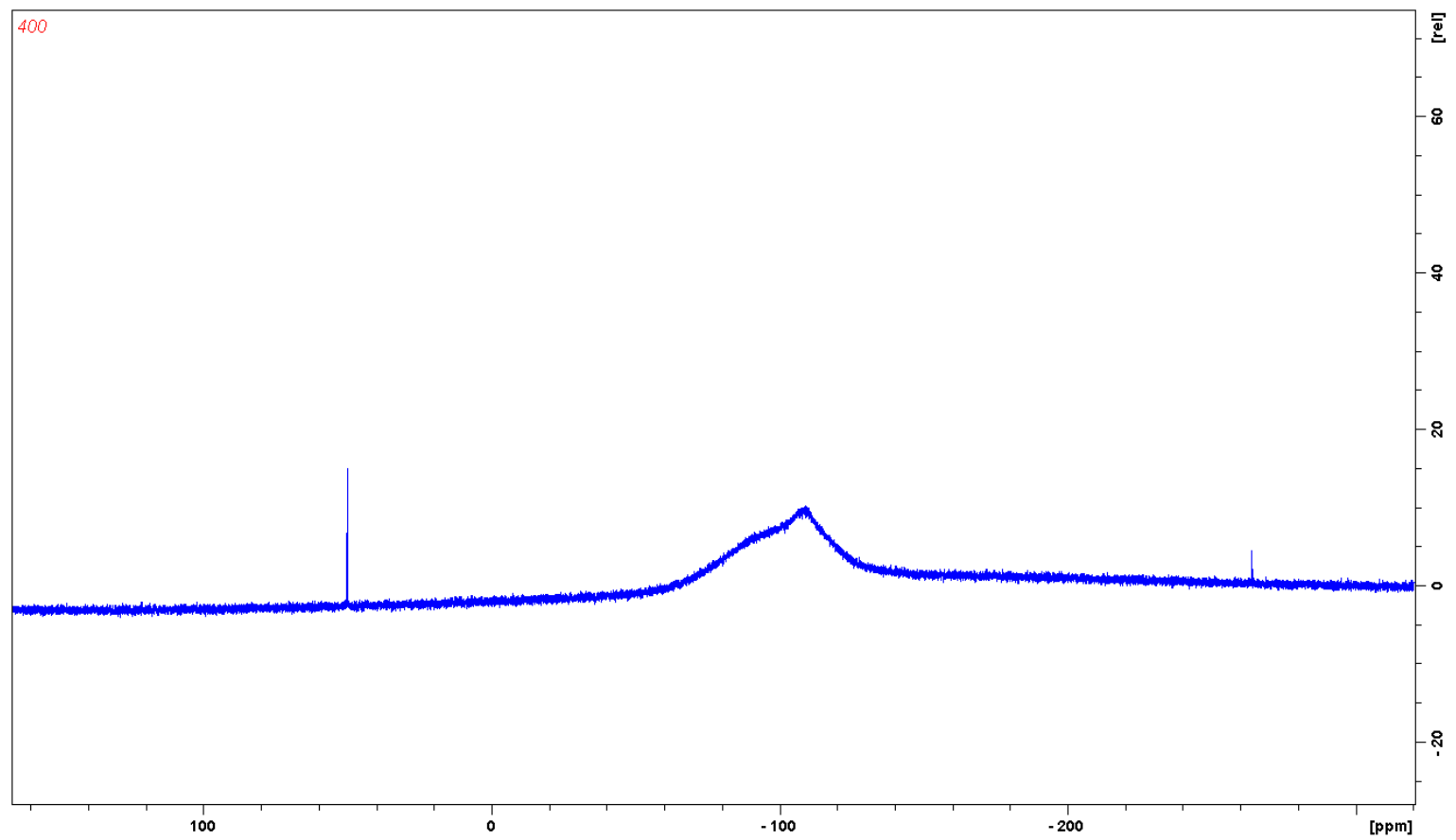

Figure S7. ${ }^{29} \mathrm{Si}\left\{{ }^{1} \mathrm{H}\right\}$ NMR spectrum of compound 3 (99.49 MHz, $\left.\mathrm{D}_{8}-\mathrm{THF}, 298 \mathrm{~K}\right)$. 


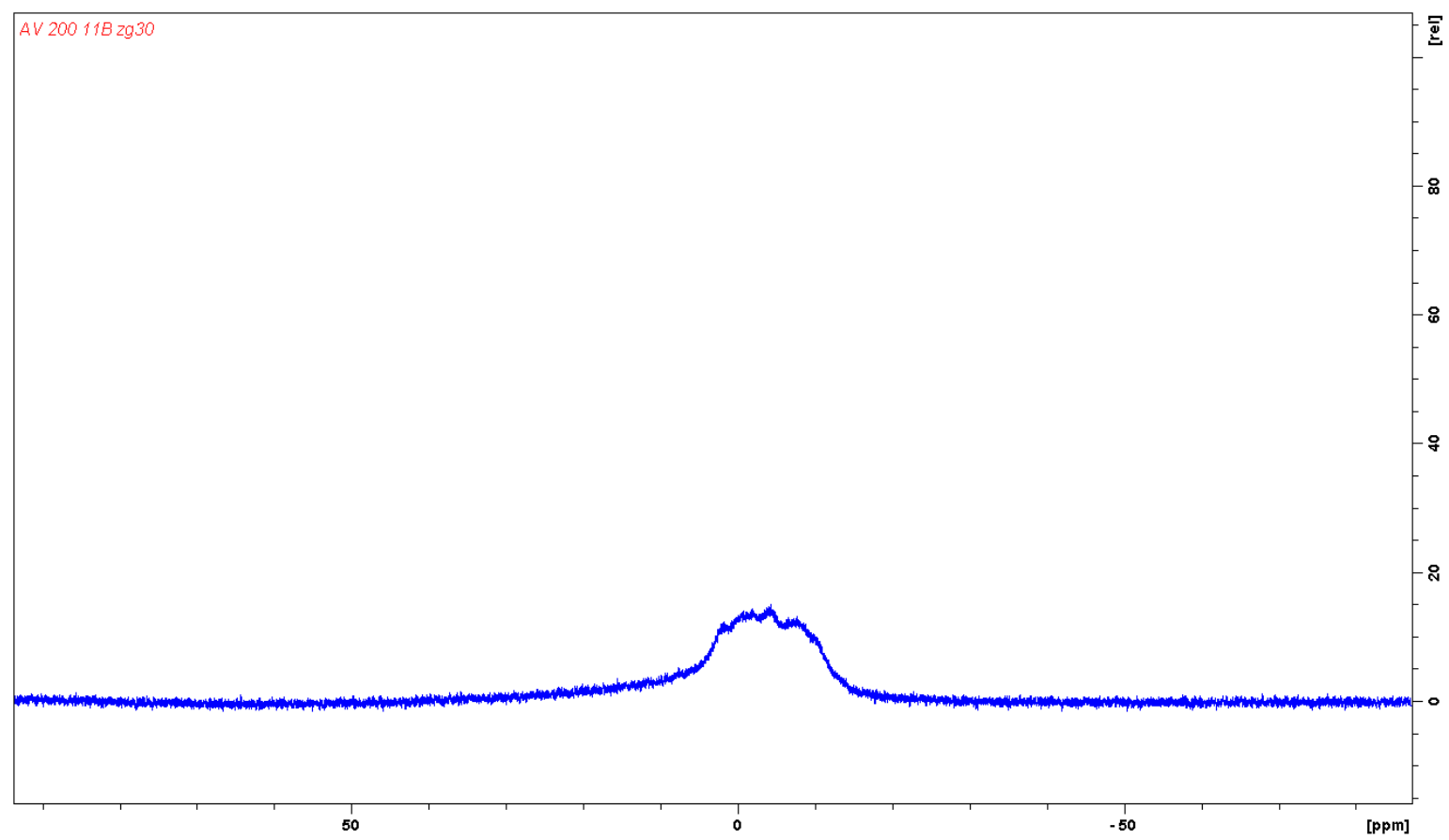

Figure S8. ${ }^{11} \mathrm{~B}\left\{{ }^{1} \mathrm{H}\right\}$ NMR spectrum of compound $3\left(64 \mathrm{MHz}, \mathrm{D}_{8}-\mathrm{THF}, 298 \mathrm{~K}\right)$.

\section{Synthesis of 4}

To a cooled $\left(-20^{\circ} \mathrm{C}\right)$ solution of $3(0.14 \mathrm{~g}, 0.20 \mathrm{mmol})$ in $5 \mathrm{~mL}$ THF was added dropwise a potassium naphthalenide THF solution prepared by stirring $7.9 \mathrm{mg}$ potassium and $26.0 \mathrm{mg}$ naphthalene in $5 \mathrm{~mL}$ THF at room temperature overnight. The reaction solution was allowed to warm to room temperature and a crystalline precipitate formed gradually. After stirring for $1 \mathrm{~h}$, volatiles were removed under vacuum and the residue was washed with $\mathrm{Et}_{2} \mathrm{O}(2 \times 8 \mathrm{~mL})$ to afford compound 4 as a red powder $(0.17 \mathrm{~g}, 94 \%$ isolated yield). Single crystals of 4 suitable for X-ray diffraction analysis were obtained from a resulting reaction solution without stirring at room temperature. M.p. $248^{\circ} \mathrm{C}$ (decomp.). Elemental analysis calcd for $\mathrm{C}_{80} \mathrm{H}_{144} \mathrm{~N}_{8} \mathrm{~B}_{20} \mathrm{Si}_{6} \mathrm{~K}_{2} \mathrm{O}_{4}$ : C 55.07, H 8.32, N 6.42; found: C 54.83, H 7.98, N 6.25; IR (cm $\left.{ }^{-1}\right): 2968$ (w), 2930 (w), 2906 (w), 2968 (w), 2464 (m), 2426 (m), 2378 (w), 1518 (w), 1469 (w), 1446 (w), 1406 (m), $1395(\mathrm{~m}), 1366$ (m), 1264 (w), $1200(\mathrm{~m}), 1140$ (w), 1073 (w), 1024 (w), 934 (w), $796(\mathrm{~m}), 751$ (s), 709 (s), $629(\mathrm{~m}), 617(\mathrm{~m}), 580(\mathrm{w}), 577(\mathrm{w})$. 


\section{A4. Crystallographic data}

Table S1. Crystal data and structure refinement for 3.

Empirical formula

Formula weight

Temperature

Wavelength

Crystal system

Space group

Unit cell dimensions

Volume

Z

Density (calculated)

Absorption coefficient

$\mathrm{F}(000)$

Crystal size

Theta range for data collection

Index ranges

Reflections collected

Independent reflections

Completeness to theta $=67.498^{\circ}$

Absorption correction

Max. and min. transmission

Refinement method

Data / restraints / parameters

Goodness-of-fit on $\mathrm{F}^{2}$

Final R indices [I $>2 \operatorname{sigma}(\mathrm{I})]$

$\mathrm{R}$ indices (all data)

Extinction coefficient

Largest diff. peak and hole
C36 H66 B10 N4 O Si3

763.29

150(2) K

$1.54184 \AA$

Triclinic

P-1

$\mathrm{a}=11.8111(3) \AA \quad \alpha=109.644(3)^{\circ}$.

$\mathrm{b}=13.9379(5) \AA \quad \beta=100.899(3)^{\circ}$.

$\mathrm{c}=16.6307(5) \AA \quad \gamma=108.196(3)^{\circ}$.

2313.42(14) $\AA^{3}$

2

$1.096 \mathrm{Mg} / \mathrm{m}^{3}$

$1.176 \mathrm{~mm}^{-1}$

820

$0.230 \times 0.090 \times 0.060 \mathrm{~mm}^{3}$

2.987 to $67.498^{\circ}$.

$-14<=\mathrm{h}<=12,-16<=\mathrm{k}<=15,-19<=\mathrm{l}<=19$

15835

$8327[\mathrm{R}($ int $)=0.0262]$

$99.9 \%$

Semi-empirical from equivalents

1.00000 and 0.42356

Full-matrix least-squares on $\mathrm{F}^{2}$

8327 / 0 / 501

1.024

$\mathrm{R} 1=0.0398, \mathrm{wR} 2=0.1022$

$\mathrm{R} 1=0.0513, \mathrm{wR} 2=0.1112$

$\mathrm{n} / \mathrm{a}$

0.357 and -0.279 e. $\AA^{-3}$ 


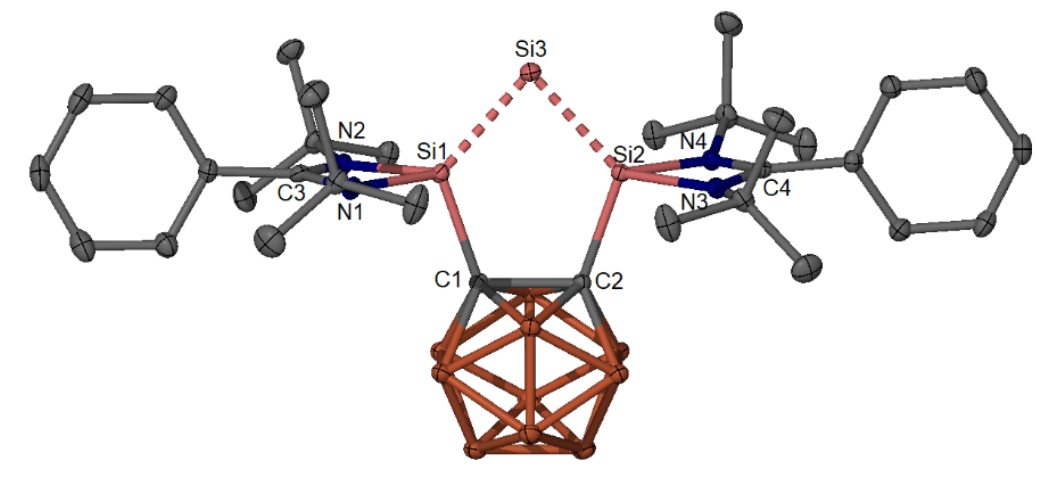

Figure S9. Molecular structure of compound 3. Thermal ellipsoids are drawn at $30 \%$ probability level. $\mathrm{H}$ atoms are omitted for clarity.

Table S2. Selected interatomic distances $[\AA]$ and angles $\left[^{\circ}\right]$ for compound 3.

\begin{tabular}{|ll|lr|}
\hline \multicolumn{3}{|c|}{ Bond distances } & \multicolumn{2}{c|}{ Bond angels } \\
\hline Si1-N2 & $1.858(1)$ & N2-Si1-N1 & $70.48(6)$ \\
Si1-N1 & $1.879(1)$ & N2-Si1-C1 & $105.24(7)$ \\
Si1-C1 & $1.941(2)$ & N1-Si1-C1 & $104.26(7)$ \\
Si1-Si3 & $2.2272(6)$ & N2-Si1-Si3 & $121.41(5)$ \\
Si1-C3 & $2.315(2)$ & N1-Si1-Si3 & $125.38(5)$ \\
Si2-N3 & $1.865(2)$ & C1-Si1-Si3 & $119.44(5)$ \\
Si2-N4 & $1.869(1)$ & C1-Si1-C3 & $110.35(7)$ \\
Si2-C2 & $1.942(2)$ & Si3-Si1-C3 & $130.16(5)$ \\
Si2-Si3 & $2.2225(6)$ & N3-Si2-N4 & $70.51(6)$ \\
Si2-C4 & $2.315(2)$ & N3-Si2-C2 & $104.83(7)$ \\
N2-C3 & $1.340(2)$ & N4-Si2-C2 & $103.99(7)$ \\
N3-C4 & $1.338(2)$ & N3-Si2-Si3 & $123.05(5)$ \\
N1-C3 & $1.336(2)$ & N4-Si2-Si3 & $124.14(5)$ \\
N4-C4 & $1.338(2)$ & C2-Si2-Si3 & $119.60(5)$ \\
C1-C2 & $1.674(2)$ & C2-Si2-C4 & $109.58(7)$ \\
& & Si3-Si2-C4 & $130.82(5)$ \\
& & Si2-Si3-Si1 & $82.75(2)$ \\
\hline
\end{tabular}




\begin{tabular}{|l|ll|}
\hline & C3-N2-Si1 & $91.3(1)$ \\
& C4-N3-Si2 & $91.1(1)$ \\
& C3-N1-Si1 & $90.5(1)$ \\
& C4-N4-Si2 & $90.9(1)$ \\
\hline
\end{tabular}


Table S3. Crystal data and structure refinement for 4 .

Empirical formula

Formula weight

Temperature

Wavelength

Crystal system

Space group

Unit cell dimensions

Volume

Z

Density (calculated)

Absorption coefficient

$\mathrm{F}(000)$

Crystal size

Theta range for data collection

Index ranges

Reflections collected

Independent reflections

Completeness to theta $=67.487^{\circ}$

Absorption correction

Max. and min. transmission

Refinement method

Data / restraints / parameters

Goodness-of-fit on $\mathrm{F}^{2}$

Final $\mathrm{R}$ indices [I $>2 \operatorname{sigma}(\mathrm{I})]$

$\mathrm{R}$ indices (all data)

Extinction coefficient

Largest diff. peak and hole
C80 H144 B20 K2 N8 O4 Si6

1744.96

150(2) K

$1.54184 \AA$

Monoclinic

$\mathrm{C} 2 / \mathrm{c}$

$\mathrm{a}=20.6665(4) \AA \quad \alpha=90^{\circ}$.

$\mathrm{b}=20.8148(3) \AA \quad \beta=104.302(2)^{\circ}$.

$\mathrm{c}=26.4814(5) \AA \quad \gamma=90^{\circ}$.

11038.4(4) $\AA^{3}$

4

$1.050 \mathrm{Mg} / \mathrm{m}^{3}$

$1.715 \mathrm{~mm}^{-1}$

3736

$0.310 \times 0.160 \times 0.070 \mathrm{~mm}^{3}$

3.062 to $67.487^{\circ}$.

$-24<=\mathrm{h}<=20,-24<=\mathrm{k}<=24,-31<=1<=30$

21198

$9914[\mathrm{R}($ int $)=0.0356]$

$99.6 \%$

Semi-empirical from equivalents

1.00000 and 0.79968

Full-matrix least-squares on $\mathrm{F}^{2}$

9914 / 447 / 700

1.037

$\mathrm{R} 1=0.0531, \mathrm{wR} 2=0.1338$

$\mathrm{R} 1=0.0764, \mathrm{wR} 2=0.1581$

$\mathrm{n} / \mathrm{a}$

0.905 and -0.574 e. $\AA^{-3}$ 


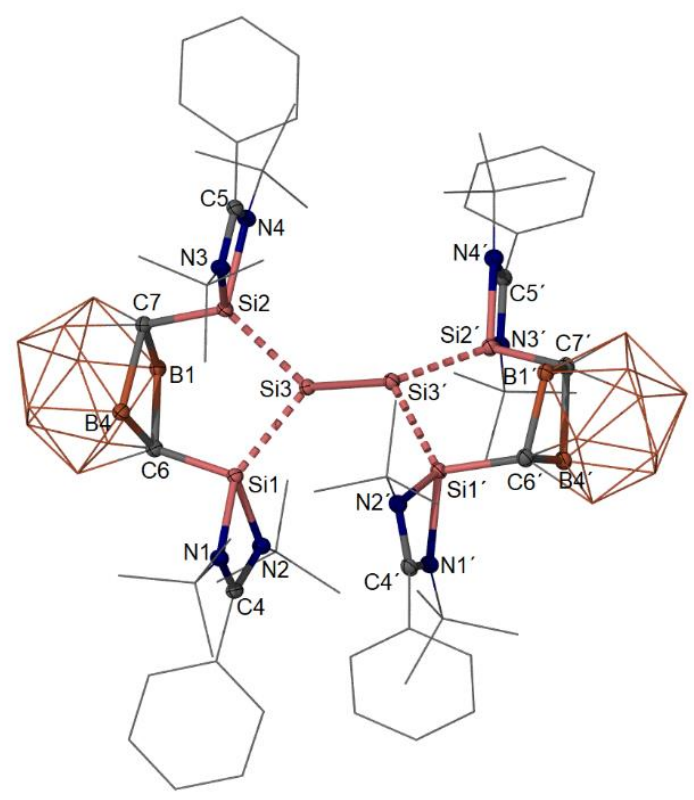

Figure S10. Molecular structure of the dianion of compound 4 (The carborane moiety is disordered over two orientations with an occupancy ratio of 0.50:0.50. One of the tert-butyl groups is disordered over two orientations with an occupancy ratio of 0.53:0.47. Only one set of them is depicted.). Thermal ellipsoids are drawn at $30 \%$ probability level. $\mathrm{H}$ atoms are omitted for clarity. Symmetry transformations used to generate equivalent atoms with ('): -x,y,$\mathrm{z}+3 / 2$.

Table S4. Selected interatomic distances $[\AA]$ and angles $\left[{ }^{\circ}\right]$ for the dianion of compound 4.

\begin{tabular}{|ll|lc|}
\hline \multicolumn{3}{|c|}{ Bond distances } & \multicolumn{2}{c|}{ Bond angels } \\
\hline Si1-C6 & $1.78(3)$ & C6-Si1-N2 & $108(1)$ \\
Si1-N2 & $1.857(2)$ & C6-Si1-N1 & $105(1)$ \\
Si1-N1 & $1.877(2)$ & N2-Si1-N1 & $70.2(1)$ \\
Si1-C4 & $2.306(2)$ & C6-Si1-C4 & $106(1)$ \\
Si1-Si3 & $2.3306(9)$ & C6-Si1-Si3 & $112(1)$ \\
Si2-C7 & $1.85(2)$ & N2-Si1-Si3 & $120.67(7)$ \\
Si2-N3 & $1.859(2)$ & N1-Si1-Si3 & $133.15(7)$ \\
Si2-N4 & $1.871(2)$ & C4-Si1-Si3 & $141.39(7)$ \\
Si2-C5 & $2.311(3)$ & C7-Si2-N3 & $115.6(5)$ \\
Si2-Si3 & $2.3486(9)$ & C7-Si2-N4 & $108.8(6)$ \\
Si3-Si3 & $2.365(1)$ & N3-Si2-N4 & $70.6(1)$ \\
N3-C5 & $1.343(3)$ & C7-Si2-C5 & $116.0(6)$ \\
N1-C4 & $1.330(3)$ & C7-Si2-Si3 & $112.7(5)$ \\
\hline
\end{tabular}




\begin{tabular}{|ll|lc|}
\hline N2-C4 & $1.338(3)$ & N3-Si2-Si3 & $124.31(7)$ \\
N4-C5 & $1.330(4)$ & N4-Si2-Si3 & $117.18(8)$ \\
B1-C7 & $1.71(2)$ & C5-Si2-Si3 & $130.10(7)$ \\
B1-C6 & $1.74(4)$ & Si1-Si3-Si2 & $96.97(3)$ \\
B4-C6 & $1.65(3)$ & Si1-Si3-Si3' & $127.22(2)$ \\
& & Si2-Si3-Si3' & $106.36(4)$ \\
& C5-N3-Si2 & $91.0(2)$ \\
& C4-N1-Si1 & $90.3(2)$ \\
& & C4-N2-Si1 & $90.9(2)$ \\
& C5-N4-Si2 & $90.8(2)$ \\
& N1-C4-N2 & $107.1(2)$ \\
& N4-C5-N3 & $107.5(2)$ \\
& B1-C7-Si2 & $108.5(9)$ \\
& & B1-C6-Si1 & $112(2)$ \\
\hline
\end{tabular}




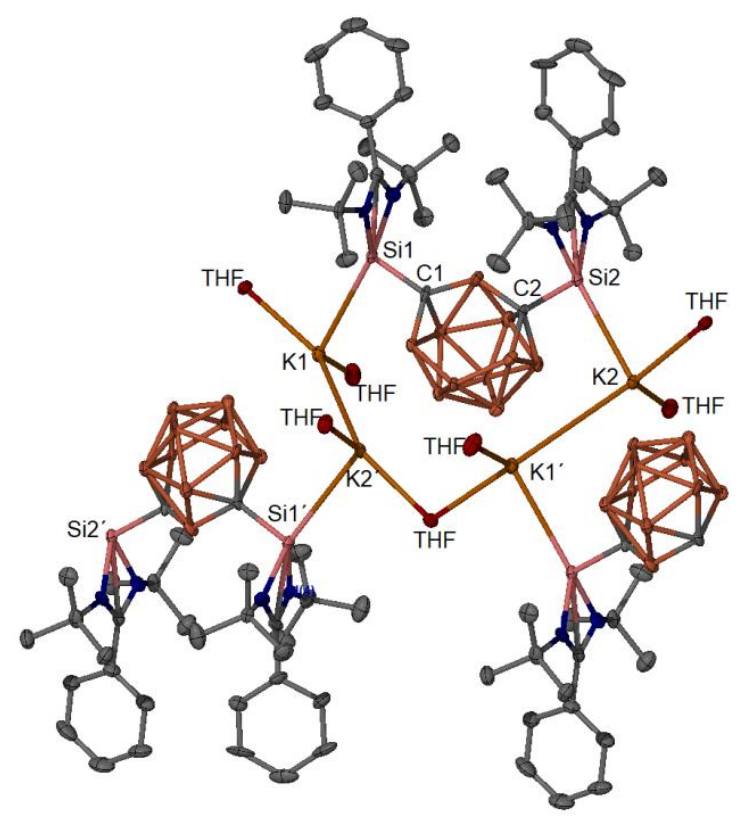

Figure S11. X-ray single crystal diffraction structure of compound 2 ( 2 crystallized with severs disorder of co-crystallized THF molecules which unfortunately did not allow to complete the stricture refinement. Only part of the one-dimensional polymeric structure formed by coordination of bis-NHSi-silicon with potassium cations is depicted). Thermal ellipsoids are drawn at 50\% probability level. $\mathrm{H}$ atoms and co-crystallized THF molecules are omitted for clarity. 


\section{B. Computational Section}

All quantum chemical calculations were carried out using Gaussian 09.D01 ${ }^{4}$. The geometries of all compounds were optimized at the B3PW915-D $3^{6}$ level of theory with $6-311+G(d, p)$ basis set for Si and $6-31 G(d, p)$ for all other atoms. ${ }^{7}$ Analytical frequencies were computed to verify the stationary points. To account for the solvent effect of THF, the polarizable Continuum Model (PCM) was used. Thermochemistry at $298.15 \mathrm{~K}$. NBO analysis was carried out using the NBO 6.0 software. ${ }^{8}$
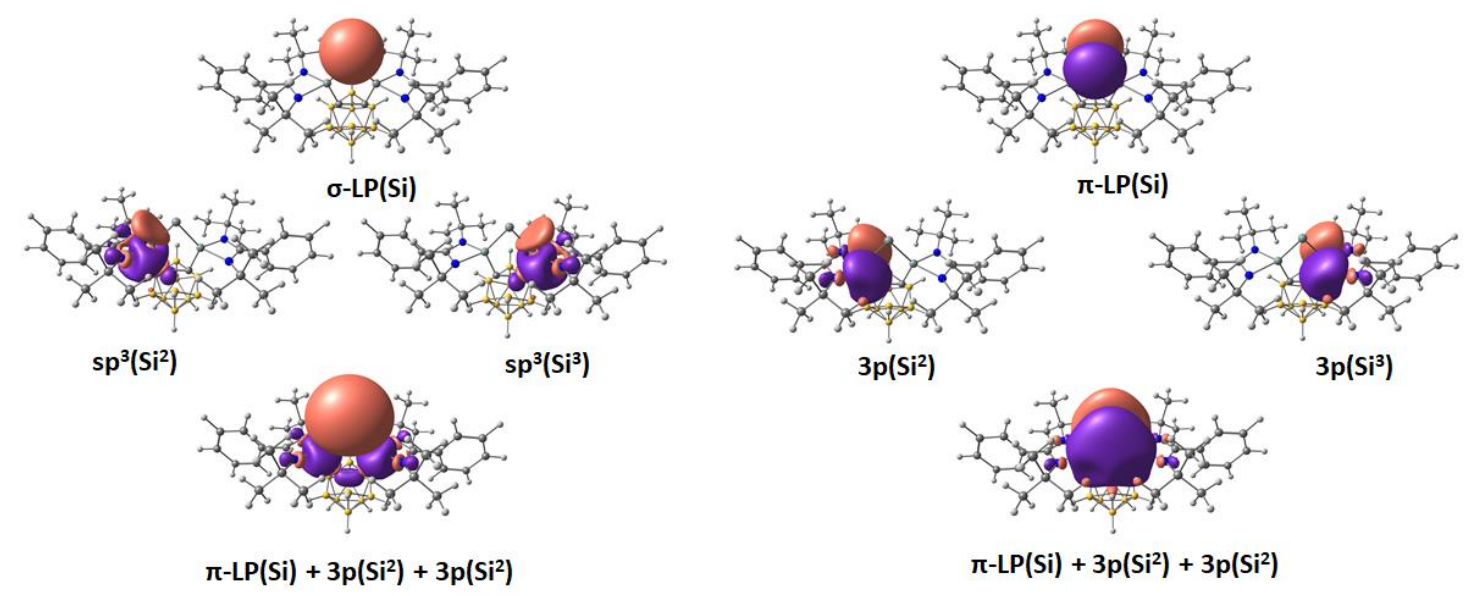

Figure S12. NBO analysis of composition of HOMO and HOMO-1 of $\mathbf{3}$.

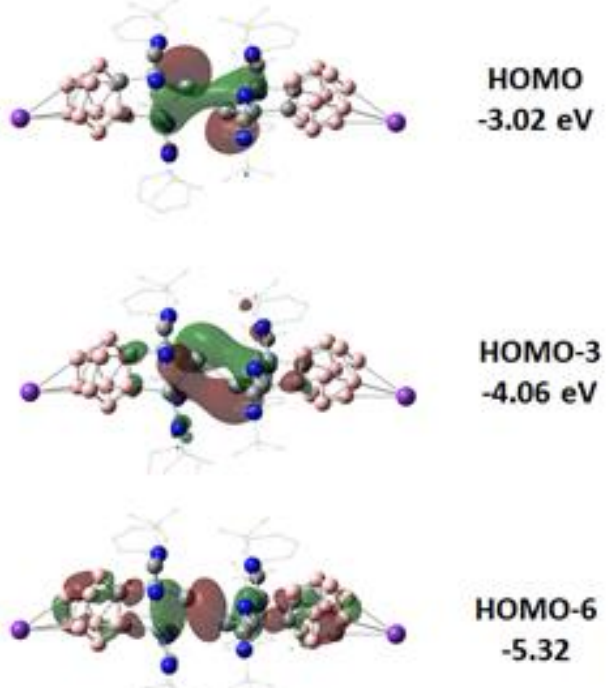

Figure S13. Frontier molecular orbitals of 4. HOMO-6 represents the $\sigma$-bond between the Si$\mathrm{Si}$. HOMO-3, HOMO correspond to the lone pairs of the cetral Si-Si. 


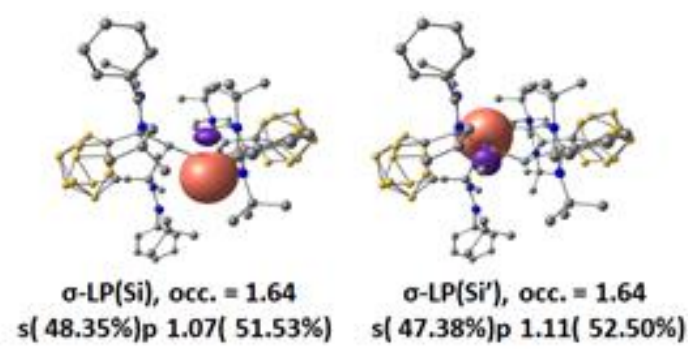

Figure S14. NBOs of 4 representing the lone pairs of the Si-Si fragment.

Table S5. NPA charges of central Si, potassium (K), amidinatosilylene (AMD-Si) and carborane (CBR) fragments in $\mathbf{3}$ and $\mathbf{4}$.

\begin{tabular}{|l|c|c|c|c|c|c|}
\hline & $\mathbf{q}(\mathbf{S i})$ & 2 $\sum \mathbf{q}(\mathbf{A M D S i})$ & $\mathbf{\Sigma q ( C B R )}$ & $\mathbf{q ( K )}$ & $\begin{array}{c}\text { WBI } \\
\text { (AMDSi-Si) }\end{array}$ & WBI(Si-Si) \\
\hline $\mathbf{3}$ & -0.55 & 1.97 & -1.42 & - & 1.44 & - \\
\hline $\mathbf{4}$ & -0.71 & 2.83 & -3.10 & 0.98 & 1.08 & 0.97 \\
& -0.72 & 2.83 & -3.10 & 0.98 & & \\
\hline
\end{tabular}




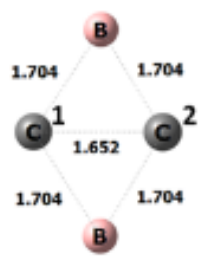

A

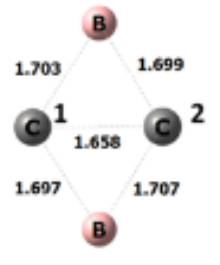

B

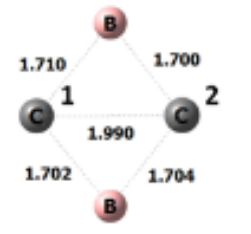

$\mathrm{TS}(\mathrm{B}-\mathrm{C})$

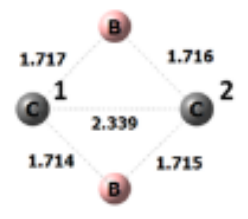

C

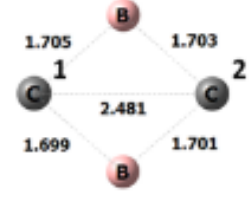

TS(C-D)

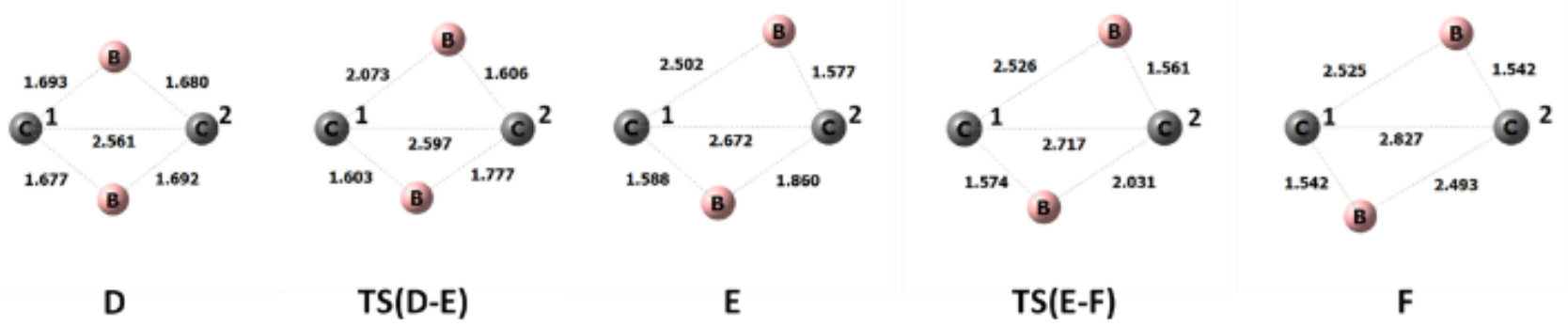

Figure S15. Geometries around the C-B-C-B facet of the carborane backbone. 

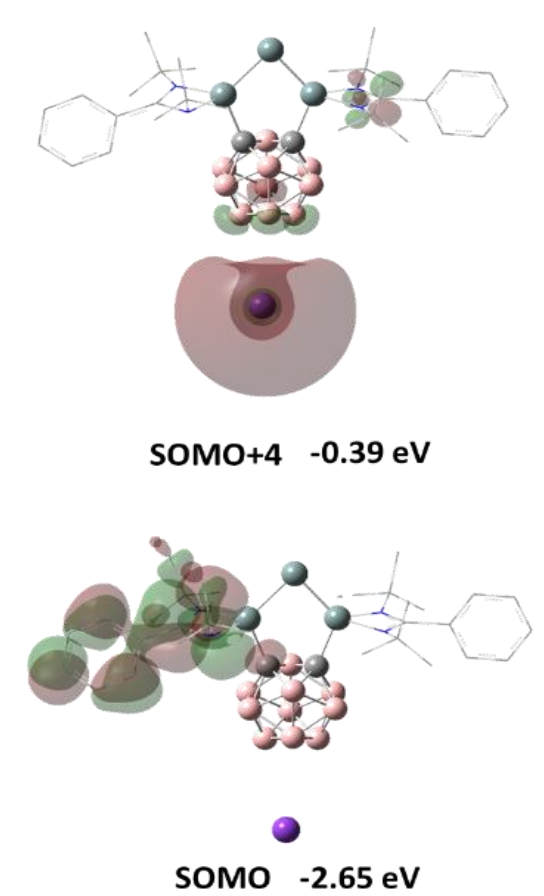

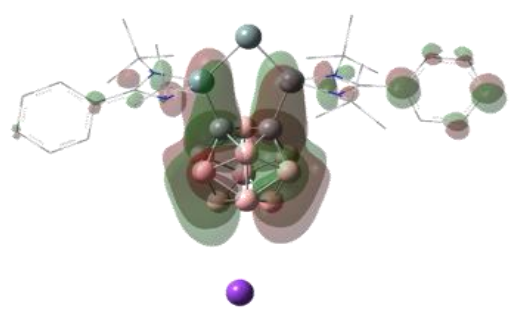

SOMO+5 $\quad-0.14 \mathrm{eV}$

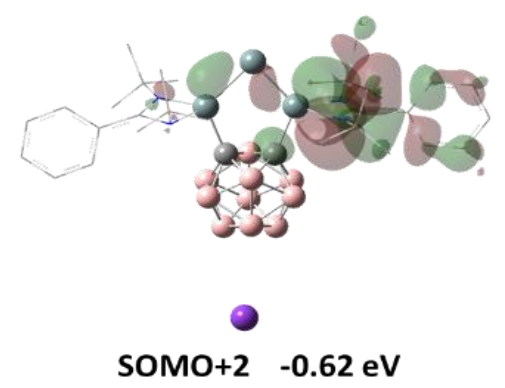

Figure S16. Selected orbitals of intermediate B.

Table S6. NPA charges and \% of spin density (in parentheses) at central $\mathrm{Si}$ atom (Si), potassium atom (K), amidinato-silylene (AMD-Si) and carborane (CBR) fragments in A-F.

\begin{tabular}{|c|c|c|cc|c|c|cc|c|c|}
\hline & A & B & TS(B-C) & C & TS(C-D) & D & TS(D-E) & E & TS(E-F) & F \\
\hline \multirow{2}{*}{$\mathrm{Si}$} & -0.58 & -0.64 & -0.65 & -0.72 & -0.48 & -0.40 & -0.40 & -0.40 & -0.40 & -0.40 \\
& $(0.1 \%)$ & $(0.2 \%)$ & $(0.9 \%)$ & $(0.5 \%)$ & $(49.8 \%)$ & $(93.6 \%)$ & $(93.3 \%)$ & $(92.9 \%)$ & $(93.0 \%)$ & $(93.7 \%)$ \\
\hline \multirow{2}{*}{ AMDSi } & +1.01 & +0.74 & +0.87 & +1.03 & +1.18 & +1.22 & +1.21 & +1.20 & +1.20 & +1.20 \\
& $(0.2 \%)$ & $(98.5 \%)$ & $(67.7 \%)$ & $(1.1 \%)$ & $(1.8 \%)$ & $(3.0 \%)$ & $(2.5 \%)$ & $(1.5 \%)$ & $(1.8 \%)$ & $(2.9 \%)$ \\
& +1.01 & +0.93 & +0.98 & +1.03 & +1.18 & +1.22 & +1.21 & +1.21 & +1.21 & +1.20 \\
& $(0.2 \%)$ & $(0.2 \%)$ & $(1.1 \%)$ & $(1.1 \%)$ & $(1.8 \%)$ & $(2.4 \%)$ & $(3.2 \%)$ & $(4.9 \%)$ & $(4.8 \%)$ & $(3.3 \%)$ \\
\hline CBR & -1.44 & -1.50 & -1.57 & -2.33 & -2.85 & -3.01 & -3.01 & -3.00 & -3.00 & -2.99 \\
& $(1.1 \%)$ & $(1.0 \%)$ & $(30.2 \%)$ & $(97.2 \%)$ & $(46.5 \%)$ & $(1.0 \%)$ & $(0.9 \%)$ & $(0.7 \%)$ & $(0.4 \%)$ & $(0.0 \%)$ \\
\hline $\mathrm{K}$ & +0.01 & +0.46 & +0.37 & +0.98 & +0.99 & +0.98 & +0.98 & +0.99 & +0.99 & +0.99 \\
& $(98.5 \%)$ & $(0.1 \%)$ & $(0.1 \%)$ & $(0.1 \%)$ & $(0.0 \%)$ & $(0.0 \%)$ & $(0.0 \%)$ & $(0.0 \%)$ & $(0.0 \%)$ & $(0.0 \%)$ \\
\hline
\end{tabular}




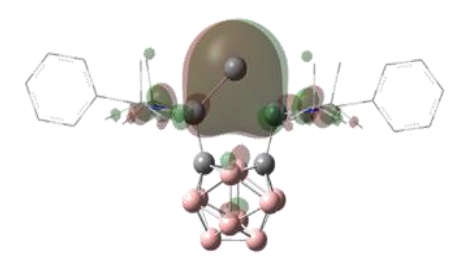

-

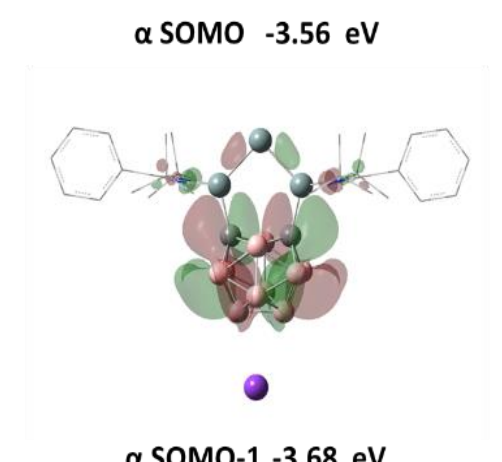

$\alpha$ SOMO-1 -3.68 eV

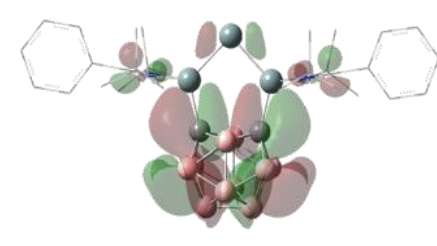

-

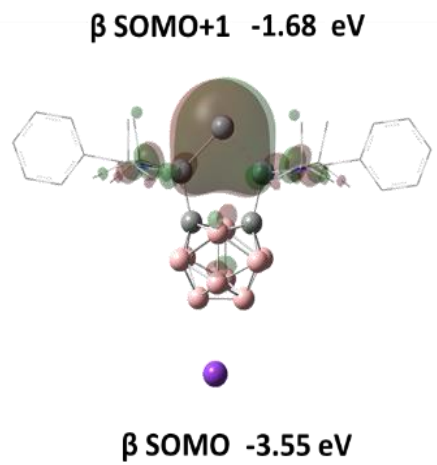

Figure S17. Selected orbitals of intermediate $\mathbf{C}$.
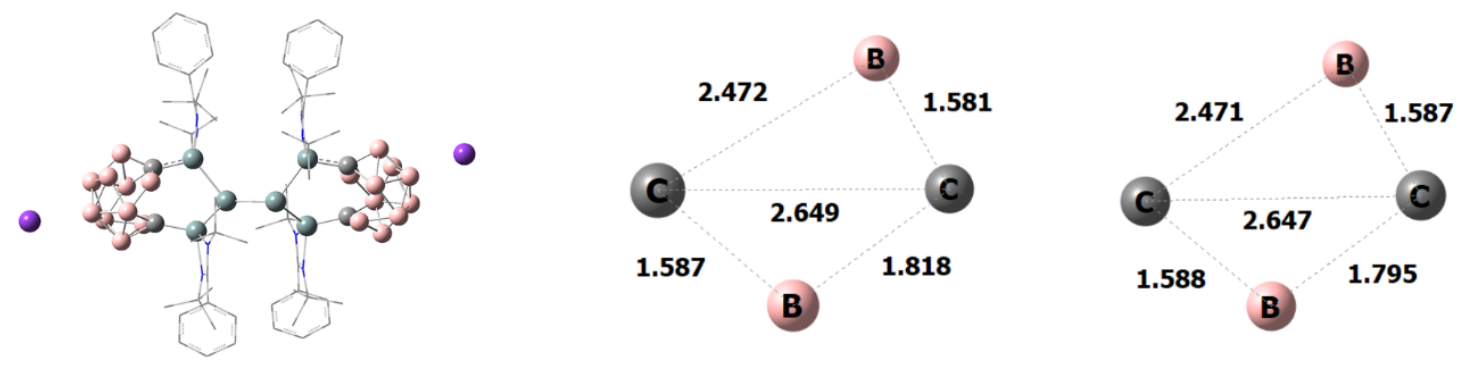

Figure S18. Optimized structure of compound 4 and geometries around the C-B-C-B facet of the carborane backbones. 


\section{Calculated cartesian coordinates and energies of 3}

$\mathrm{E}(\mathrm{RB} 3 \mathrm{PW} 91)=$

Sum of electronic and zero-point Energies=

Sum of electronic and thermal Energies=

Sum of electronic and thermal Enthalpies= Sum of electronic and thermal Free Energies $=\quad-2588.098478 E_{h}$
$-2588.896643 E_{h}$ $-2588.020564 E_{h}$ $-2587.971723 \mathrm{Eh}_{\mathrm{h}}$ $-2587.970778 E_{h}$

\begin{tabular}{|c|c|c|c|}
\hline Si & -1.44410 & -0.00003 & -0.51805 \\
\hline $\mathrm{Si}$ & 1.44405 & -0.00004 & -0.51813 \\
\hline Si & -0.00008 & -0.00003 & -2.22035 \\
\hline$N$ & -2.97895 & 1.07895 & -0.42731 \\
\hline$N$ & 2.97898 & -1.07894 & -0.42764 \\
\hline$N$ & -2.97901 & -1.07888 & -0.42745 \\
\hline$N$ & 2.97889 & 1.07891 & -0.42739 \\
\hline C & 3.32078 & 2.48718 & -0.70960 \\
\hline C & -5.24652 & 0.00012 & -0.39390 \\
\hline C & -3.76597 & 0.00006 & -0.43962 \\
\hline C & 3.76591 & 0.00002 & -0.43971 \\
\hline C & 3.32102 & -2.48715 & -0.70996 \\
\hline C & -0.82651 & -0.00009 & 1.32116 \\
\hline C & -8.02701 & 0.00029 & -0.21297 \\
\hline $\mathrm{H}$ & -9.11084 & 0.00034 & -0.14255 \\
\hline C & 5.24646 & 0.00013 & -0.39392 \\
\hline C & -3.32104 & -2.48710 & -0.70973 \\
\hline C & 0.82657 & -0.00013 & 1.32111 \\
\hline C & 6.01959 & 0.00022 & -1.55664 \\
\hline $\mathrm{H}$ & 5.53590 & 0.00021 & -2.52810 \\
\hline C & -5.86434 & 0.00004 & 0.86154 \\
\hline $\mathrm{H}$ & -5.25070 & -0.00007 & 1.75744 \\
\hline C & -3.32081 & 2.48725 & -0.70930 \\
\hline C & -6.01951 & 0.00030 & -1.55673 \\
\hline $\mathrm{H}$ & -5.53569 & 0.00035 & -2.52813 \\
\hline C & -2.10440 & 3.33602 & -0.33558 \\
\hline $\mathrm{H}$ & -1.92453 & 3.30317 & 0.74083 \\
\hline $\mathrm{H}$ & -2.28198 & 4.37615 & -0.62454 \\
\hline $\mathrm{H}$ & -1.21035 & 2.98007 & -0.85686 \\
\hline C & 2.10443 & 3.33605 & -0.33593 \\
\hline $\mathrm{H}$ & 1.21034 & 2.98012 & -0.85717 \\
\hline $\mathrm{H}$ & 2.28206 & 4.37616 & -0.62494 \\
\hline $\mathrm{H}$ & 1.92457 & 3.30327 & 0.74049 \\
\hline C & -4.52446 & 2.97115 & 0.10948 \\
\hline $\mathrm{H}$ & -5.46449 & 2.52913 & -0.22429 \\
\hline $\mathrm{H}$ & -4.61043 & 4.05724 & 0.00466 \\
\hline $\mathrm{H}$ & -4.38510 & 2.73929 & 1.16955 \\
\hline B & -1.43912 & 0.88221 & 2.64746 \\
\hline $\mathrm{H}$ & -2.44959 & 1.48897 & 2.49559 \\
\hline B & 1.43932 & -0.88295 & 2.64703 \\
\hline $\mathrm{H}$ & 2.44981 & -1.48961 & 2.49483 \\
\hline C & 7.41023 & 0.00036 & -1.46281 \\
\hline $\mathrm{H}$ & 8.01036 & 0.00044 & -2.36795 \\
\hline B & 0.00005 & -1.42041 & 1.77278 \\
\hline
\end{tabular}




\begin{tabular}{|c|c|c|c|}
\hline $\mathrm{H}$ & 0.00003 & -2.33228 & 1.03122 \\
\hline 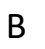 & -1.43914 & -0.88289 & 2.64714 \\
\hline$H$ & -2.44965 & -1.48954 & 2.49500 \\
\hline C & 3.58983 & 2.63145 & -2.21544 \\
\hline $\mathrm{H}$ & 4.47415 & 2.06046 & -2.51143 \\
\hline $\mathrm{H}$ & 3.76674 & 3.68132 & -2.47134 \\
\hline $\mathrm{H}$ & 2.72882 & 2.27054 & -2.78703 \\
\hline B & 0.00009 & 1.41999 & 1.77324 \\
\hline $\mathrm{H}$ & 0.00011 & 2.33212 & 1.03199 \\
\hline C & 5.86413 & 0.00020 & 0.86161 \\
\hline $\mathrm{H}$ & 5.25037 & 0.00017 & 1.75743 \\
\hline C & -7.25242 & 0.00012 & 0.94824 \\
\hline $\mathrm{H}$ & -7.73080 & 0.00006 & 1.92317 \\
\hline B & 0.00016 & 1.44433 & 3.53667 \\
\hline $\mathrm{H}$ & 0.00020 & 2.48250 & 4.11911 \\
\hline C & 4.52442 & 2.97115 & 0.10913 \\
\hline $\mathrm{H}$ & 4.38503 & 2.73940 & 1.16923 \\
\hline $\mathrm{H}$ & 4.61041 & 4.05723 & 0.00421 \\
\hline $\mathrm{H}$ & 5.46444 & 2.52908 & -0.22458 \\
\hline C & -7.41015 & 0.00038 & -1.46307 \\
\hline $\mathrm{H}$ & -8.01017 & 0.00051 & -2.36829 \\
\hline C & -3.58988 & 2.63173 & -2.21513 \\
\hline $\mathrm{H}$ & -2.72893 & 2.27080 & -2.78681 \\
\hline $\mathrm{H}$ & -3.76668 & 3.68166 & -2.47089 \\
\hline $\mathrm{H}$ & -4.47426 & 2.06088 & -2.51116 \\
\hline C & 2.10478 & -3.33619 & -0.33635 \\
\hline $\mathrm{H}$ & 1.92500 & -3.30367 & 0.74008 \\
\hline $\mathrm{H}$ & 2.28249 & -4.37622 & -0.62560 \\
\hline $\mathrm{H}$ & 1.21063 & -2.98024 & -0.85745 \\
\hline C & -2.10473 & -3.33610 & -0.33625 \\
\hline $\mathrm{H}$ & -1.21066 & -2.98014 & -0.85747 \\
\hline $\mathrm{H}$ & -2.28247 & -4.37613 & -0.62549 \\
\hline $\mathrm{H}$ & -1.92483 & -3.30356 & 0.74016 \\
\hline C & -3.59017 & -2.63123 & -2.21557 \\
\hline $\mathrm{H}$ & -4.47446 & -2.06016 & -2.51148 \\
\hline $\mathrm{H}$ & -3.76716 & -3.68107 & -2.47153 \\
\hline $\mathrm{H}$ & -2.72917 & -2.27033 & -2.78720 \\
\hline C & 8.02692 & 0.00040 & -0.21263 \\
\hline $\mathrm{H}$ & 9.11075 & 0.00051 & -0.14208 \\
\hline B & -0.89140 & -0.00059 & 4.08977 \\
\hline $\mathrm{H}$ & -1.53719 & -0.00075 & 5.09013 \\
\hline B & 0.00011 & -1.44537 & 3.53618 \\
\hline $\mathrm{H}$ & 0.00010 & -2.48375 & 4.11823 \\
\hline B & 1.43934 & 0.88216 & 2.64733 \\
\hline $\mathrm{H}$ & 2.44983 & 1.48887 & 2.49532 \\
\hline C & 3.59001 & -2.63131 & -2.21582 \\
\hline $\mathrm{H}$ & 2.72893 & -2.27048 & -2.78736 \\
\hline $\mathrm{H}$ & 3.76701 & -3.68116 & -2.47174 \\
\hline $\mathrm{H}$ & 4.47425 & -2.06024 & -2.51184 \\
\hline C & -4.52471 & -2.97104 & 0.10901 \\
\hline $\mathrm{H}$ & -4.38530 & -2.73936 & 1.16912 \\
\hline
\end{tabular}




$\begin{array}{llll}\mathrm{H} & -4.61077 & -4.05711 & 0.00404 \\ \mathrm{H} & -5.46472 & -2.52891 & -0.22466 \\ \mathrm{~B} & 0.89171 & -0.00062 & 4.08970 \\ \mathrm{H} & 1.53756 & -0.00076 & 5.09002 \\ \mathrm{C} & 4.52478 & -2.97105 & 0.10868 \\ \mathrm{H} & 5.46474 & -2.52894 & -0.22514 \\ \mathrm{H} & 4.61082 & -4.05713 & 0.00380 \\ \mathrm{H} & 4.38548 & -2.73926 & 1.16878 \\ \mathrm{C} & 7.25220 & 0.00032 & 0.94848 \\ \mathrm{H} & 7.73045 & 0.00036 & 1.92348\end{array}$

\section{Calculated cartesian coordinates and energies of $A$}

$\begin{array}{ll}\text { E(UB3PW91) = } & -3188.754384 E_{h} \\ \text { Sum of electronic and zero-point Energies }= & -3187.878463 E_{h} \\ \text { Sum of electronic and thermal Energies= } & -3187.827129 E_{h} \\ \text { Sum of electronic and thermal Enthalpies }= & -3187.826184 E_{h} \\ \text { Sum of electronic and thermal Free Energies= } & -3187.963358 E_{h} \\ \text { Number of imaginary frequencies: } 0 & \end{array}$

$\begin{array}{llll}\text { K } & -0.01858 & 7.34420 & -0.50690 \\ \mathrm{Si} & -1.41911 & -1.16836 & 0.11611 \\ \mathrm{Si} & 1.53665 & -1.14801 & 0.09925 \\ \mathrm{Si} & 0.07313 & -2.75749 & 0.62393 \\ \mathrm{C} & -3.31734 & -2.09262 & -2.18159 \\ \mathrm{C} & -3.25366 & -0.58284 & 2.56464 \\ \mathrm{C} & 2.19751 & -1.98262 & -3.13895 \\ \mathrm{H} & 1.98707 & -0.95308 & -3.43532 \\ \mathrm{H} & 2.38986 & -2.56847 & -4.04213 \\ \mathrm{H} & 1.31535 & -2.39712 & -2.64103 \\ \mathrm{C} & -5.21043 & -1.03572 & 0.12246 \\ \mathrm{C} & -7.21474 & 0.25417 & -0.25628 \\ \mathrm{H} & -7.69240 & 1.18588 & -0.54382 \\ \mathrm{C} & -2.03370 & 0.04247 & 3.24241 \\ \mathrm{H} & -1.86906 & 1.05984 & 2.88093 \\ \mathrm{H} & -2.19646 & 0.08227 & 4.32315 \\ \mathrm{H} & -1.13509 & -0.55226 & 3.05122 \\ \mathrm{C} & -4.51643 & -1.43338 & -2.87484 \\ \mathrm{H} & -4.36221 & -0.35433 & -2.96860 \\ \mathrm{H} & -4.61794 & -1.85488 & -3.87959 \\ \mathrm{H} & -5.45348 & -1.60635 & -2.34387 \\ \mathrm{C} & 4.59875 & -1.36944 & -2.93254 \\ \mathrm{H} & 5.54550 & -1.52528 & -2.41360 \\ \mathrm{H} & 4.69376 & -1.79270 & -3.93720 \\ \mathrm{H} & 4.42591 & -0.29337 & -3.02774 \\ \mathrm{C} & 3.41962 & -2.04568 & -2.22153 \\ \mathrm{C} & -5.98226 & -2.14200 & 0.48496 \\ \mathrm{H} & -5.50195 & -3.07106 & 0.77413 \\ \mathrm{C} & 5.32437 & -0.94027 & 0.04896 \\ \mathrm{C} & -4.46009 & 0.33777 & 2.78878 \\ \mathrm{H} & -5.40096 & -0.12338 & 2.48534\end{array}$




\begin{tabular}{|c|c|c|c|}
\hline $\mathrm{H}$ & -4.53084 & 0.57066 & 3.85568 \\
\hline $\mathrm{H}$ & -4.33678 & 1.27573 & 2.23927 \\
\hline$C$ & -3.61089 & -3.56777 & -1.87209 \\
\hline $\mathrm{H}$ & -4.48583 & -3.66497 & -1.22446 \\
\hline $\mathrm{H}$ & -3.81265 & -4.11590 & -2.79788 \\
\hline $\mathrm{H}$ & -2.75132 & -4.02658 & -1.37303 \\
\hline C & -2.10825 & -2.00603 & -3.11420 \\
\hline $\mathrm{H}$ & -1.21240 & -2.40481 & -2.62803 \\
\hline $\mathrm{H}$ & -2.30110 & -2.59397 & -4.01593 \\
\hline $\mathrm{H}$ & -1.92011 & -0.97236 & -3.41140 \\
\hline C & -3.51134 & -1.97232 & 3.16634 \\
\hline $\mathrm{H}$ & -2.64820 & -2.62379 & 2.99576 \\
\hline $\mathrm{H}$ & -3.68264 & -1.89207 & 4.24460 \\
\hline $\mathrm{H}$ & -4.39404 & -2.43528 & 2.71749 \\
\hline C & -5.82653 & 0.16477 & -0.24832 \\
\hline $\mathrm{H}$ & -5.21478 & 1.01745 & -0.52624 \\
\hline C & 3.74071 & -3.51478 & -1.91056 \\
\hline $\mathrm{H}$ & 2.89527 & -3.98427 & -1.39743 \\
\hline $\mathrm{H}$ & 3.93788 & -4.06377 & -2.83684 \\
\hline $\mathrm{H}$ & 4.62603 & -3.59574 & -1.27489 \\
\hline C & 5.90646 & 0.27431 & -0.33085 \\
\hline $\mathrm{H}$ & 5.27076 & 1.11362 & -0.59557 \\
\hline C & 8.09744 & -0.69332 & -0.02199 \\
\hline $\mathrm{H}$ & 9.17881 & -0.59769 & -0.05006 \\
\hline C & 6.12713 & -2.02956 & 0.39509 \\
\hline $\mathrm{H}$ & 5.67289 & -2.96928 & 0.69177 \\
\hline C & -7.98928 & -0.85016 & 0.10436 \\
\hline $\mathrm{H}$ & -9.07282 & -0.77841 & 0.09732 \\
\hline C & 7.29196 & 0.39430 & -0.36523 \\
\hline $\mathrm{H}$ & 7.74342 & 1.33673 & -0.66010 \\
\hline B & 1.46976 & 1.56606 & -1.72882 \\
\hline $\mathrm{H}$ & 2.48055 & 1.24015 & -2.26021 \\
\hline C & -7.37304 & -2.04503 & 0.47456 \\
\hline $\mathrm{H}$ & -7.97306 & -2.90498 & 0.75624 \\
\hline C & 7.51501 & -1.90197 & 0.35813 \\
\hline $\mathrm{H}$ & 8.13919 & -2.74880 & 0.62669 \\
\hline B & 0.03567 & 0.55499 & -1.95573 \\
\hline $\mathrm{H}$ & 0.03929 & -0.43855 & -2.58261 \\
\hline$N$ & 3.06121 & -1.36709 & -0.95750 \\
\hline$N$ & -2.95384 & -1.41153 & -0.92034 \\
\hline$N$ & -2.92743 & -0.74458 & 1.13143 \\
\hline C & -3.73090 & -1.08580 & 0.11799 \\
\hline C & 3.84654 & -1.02352 & 0.06873 \\
\hline B & 0.04424 & 1.45344 & 0.74135 \\
\hline $\mathrm{H}$ & 0.05388 & 1.03836 & 1.84012 \\
\hline B & -1.40360 & 2.10481 & -0.03762 \\
\hline $\mathrm{H}$ & -2.41102 & 2.14800 & 0.59000 \\
\hline B & 1.47515 & 2.12346 & -0.05333 \\
\hline $\mathrm{H}$ & 2.48871 & 2.17994 & 0.56303 \\
\hline C & 0.86947 & 0.58496 & -0.47002 \\
\hline C & -0.78290 & 0.57400 & -0.46088 \\
\hline
\end{tabular}




$\begin{array}{lrrr}\text { B } & -1.40866 & 1.54774 & -1.71344 \\ \text { H } & -2.42072 & 1.20969 & -2.23454 \\ \text { B } & 0.02205 & 2.22327 & -2.53376 \\ \text { B } & -0.86818 & 3.19566 & -1.33328 \\ \text { B } & 0.91236 & 3.20696 & -1.34268 \\ \text { B } & 0.03056 & 3.13147 & 0.20640 \\ \text { H } & 0.01412 & 2.45288 & -3.70294 \\ \text { H } & -1.51821 & 4.14774 & -1.64444 \\ \text { H } & 1.54619 & 4.16775 & -1.66057 \\ \text { H } & 0.02917 & 4.01773 & 1.00535 \\ \text { C } & 3.39642 & -0.52704 & 2.52144 \\ \text { N } & 3.05147 & -0.69762 & 1.09371 \\ \text { C } & 3.69333 & -1.91013 & 3.11978 \\ \text { H } & 3.88291 & -1.82466 & 4.19458 \\ \text { H } & 2.84101 & -2.57980 & 2.96634 \\ \text { H } & 4.57687 & -2.35557 & 2.65503 \\ \text { C } & 2.17443 & 0.07289 & 3.21830 \\ \text { H } & 1.98480 & 1.08771 & 2.86195 \\ \text { H } & 1.28476 & -0.53884 & 3.03902 \\ \text { H } & 2.35236 & 0.11376 & 4.29663 \\ \text { C } & 4.58683 & 0.41871 & 2.72619 \\ \text { H } & 4.66795 & 0.65599 & 3.79140 \\ \text { H } & 5.53257 & -0.02399 & 2.41054 \\ \text { H } & 4.43667 & 1.35233 & 2.17606\end{array}$

\section{Calculated cartesian coordinates and energies of $B$}

$\begin{array}{ll}\text { E(UB3PW91) }= & -3188.769147 E_{h} \\ \text { Sum of electronic and zero-point Energies }= & -3187.897585 E_{h} \\ \text { Sum of electronic and thermal Energies }= & -3187.846091 E_{h} \\ \text { Sum of electronic and thermal Enthalpies }= & -3187.845147 E_{h} \\ \text { Sum of electronic and thermal Free Energies= } & -3187.981718 E_{h} \\ \text { Number of imaginary frequencies: } 0 & \end{array}$

$\begin{array}{lrrr}\text { K } & 0.57188 & 6.50806 & 0.14920 \\ \mathrm{Si} & -1.47318 & -1.08221 & -0.07923 \\ \mathrm{Si} & 1.58106 & -1.21210 & -0.07433 \\ \mathrm{Si} & -0.06858 & -2.78811 & 0.05127 \\ \mathrm{C} & -3.48485 & -1.52315 & -2.43220 \\ \mathrm{C} & -3.25461 & -0.65044 & 2.46508 \\ \mathrm{C} & 2.20113 & -1.69598 & -3.23917 \\ \mathrm{H} & 1.90996 & -0.65162 & -3.36930 \\ \mathrm{H} & 2.39487 & -2.12024 & -4.22972 \\ \mathrm{H} & 1.36351 & -2.23639 & -2.78523 \\ \mathrm{C} & -5.26388 & -0.66747 & 0.03113 \\ \mathrm{C} & -7.18946 & 0.77771 & -0.15499 \\ \mathrm{H} & -7.61149 & 1.76489 & -0.31828 \\ \mathrm{C} & -1.96922 & -0.24014 & 3.18567 \\ \mathrm{H} & -1.70242 & 0.79162 & 2.94788 \\ \mathrm{H} & -2.11284 & -0.31976 & 4.26725 \\ \mathrm{H} & -1.14003 & -0.89238 & 2.89778 \\ \mathrm{C} & -4.68256 & -0.73839 & -2.98476 \\ \mathrm{H} & -4.49919 & 0.33848 & -2.92186 \\ \mathrm{H} & -4.82038 & -1.00252 & -4.03796\end{array}$




\begin{tabular}{|c|c|c|c|}
\hline H & -5.61226 & 00 & 045 \\
\hline C & 4.51211 & -0.85462 & -2.98037 \\
\hline $\mathrm{H}$ & 5.49723 & -0.95852 & -2.52680 \\
\hline $\mathrm{H}$ & 4.60776 & -1.06237 & -4.05174 \\
\hline $\mathrm{H}$ & 4.19188 & 0.18419 & -2.85940 \\
\hline C & 3.46428 & -1.80262 & -2.37455 \\
\hline C & -6.10112 & -1.76115 & 0.26291 \\
\hline $\mathrm{H}$ & -5.67596 & -2.74681 & 0.42224 \\
\hline C & 5.24875 & -0.66307 & 0.18443 \\
\hline C & -4.35527 & 0.35950 & 2.81631 \\
\hline $\mathrm{H}$ & -5.34215 & 0.03605 & 2.48198 \\
\hline $\mathrm{H}$ & -4.39223 & 0.47856 & 3.90378 \\
\hline $\mathrm{H}$ & -4.13827 & 1.33484 & 2.37031 \\
\hline C & -3.82172 & -3.01800 & -2.32405 \\
\hline $\mathrm{H}$ & -4.67136 & -3.17980 & -1.65509 \\
\hline $\mathrm{H}$ & -4.08315 & -3.42047 & -3.30831 \\
\hline $\mathrm{H}$ & -2.95931 & -3.57044 & -1.93736 \\
\hline C & -2.30855 & -1.34207 & -3.39405 \\
\hline $\mathrm{H}$ & -1.40903 & -1.82890 & -3.00543 \\
\hline $\mathrm{H}$ & -2.55735 & -1.78984 & -4.36079 \\
\hline $\mathrm{H}$ & -2.09588 & -0.28203 & -3.54978 \\
\hline C & -3.65540 & -2.06257 & 2.91771 \\
\hline $\mathrm{H}$ & -2.86348 & -2.77691 & 2.67294 \\
\hline $\mathrm{H}$ & -3.82091 & -2.08033 & 3.99991 \\
\hline $\mathrm{H}$ & -4.57905 & -2.38223 & 2.42887 \\
\hline C & -5.80936 & 0.60439 & -0.17814 \\
\hline $\mathrm{H}$ & -5.14782 & 1.44581 & -0.36047 \\
\hline C & 3.94513 & -3.26445 & -2.41146 \\
\hline $\mathrm{H}$ & 3.13421 & -3.92496 & -2.08676 \\
\hline $\mathrm{H}$ & 4.25046 & -3.55333 & -3.42349 \\
\hline $\mathrm{H}$ & 4.7 & -3.4 & -1.74019 \\
\hline C & 5.58836 & 0.52066 & 0.90591 \\
\hline $\mathrm{H}$ & 4.78014 & 1.10407 & 1.33461 \\
\hline C & 7.95485 & 0.20462 & 0.47376 \\
\hline $\mathrm{H}$ & 8.98442 & 0.53212 & 0.58447 \\
\hline C & 6.33502 & -1.39400 & -0.37680 \\
\hline $\mathrm{H}$ & 6.14006 & -2.32864 & -0.88994 \\
\hline C & -8.02842 & -0.31466 & 0.07516 \\
\hline $\mathrm{H}$ & -9.10568 & -0.17795 & 0.09122 \\
\hline C & 6.90070 & 0.93912 & 1.04048 \\
\hline $\mathrm{H}$ & 7.11199 & 1.85832 & 1.58347 \\
\hline B & 1.51089 & 1.73461 & -1.62449 \\
\hline $\mathrm{H}$ & 2.47446 & 1.45895 & -2.25765 \\
\hline C & -7.48355 & -1.58117 & 0.28387 \\
\hline $\mathrm{H}$ & -8.13314 & -2.43274 & 0.46249 \\
\hline C & 7.64676 & -0.96616 & -0.23238 \\
\hline $\mathrm{H}$ & 8.44694 & -1.56424 & -0.66344 \\
\hline B & 0.01900 & 0.83629 & -1.90821 \\
\hline $\mathrm{H}$ & -0.05817 & -0.05054 & -2.67739 \\
\hline $\mathrm{N}$ & 3.10020 & -1.46026 & -0.99676 \\
\hline $\mathrm{N}$ & -3.05421 & -1.03950 & -1.10413 \\
\hline $\mathrm{N}$ & -2.95484 & -0.67783 & 1.02020 \\
\hline C & -3.79055 & -0.81258 & -0.01225 \\
\hline C & 3.87590 & -1.05170 & 0.09500 \\
\hline B & 0.21304 & 1.33710 & 0.87747 \\
\hline $\mathrm{H}$ & 0.27453 & 0.77540 & 1.91149 \\
\hline
\end{tabular}




$\begin{array}{lrrr}\text { B } & -1.23911 & 2.16631 & 0.28021 \\ \text { H } & -2.21365 & 2.18308 & 0.95818 \\ \text { B } & 1.62928 & 2.05438 & 0.10344 \\ \text { H } & 2.67064 & 1.99298 & 0.66442 \\ \text { C } & 0.92423 & 0.61403 & -0.48302 \\ \text { C } & -0.72906 & 0.68881 & -0.38937 \\ \text { B } & -1.35821 & 1.85612 & -1.45595 \\ \text { H } & -2.41405 & 1.65354 & -1.96067 \\ \text { B } & 0.06509 & 2.56843 & -2.25494 \\ \text { B } & -0.71210 & 3.40341 & -0.88219 \\ \text { B } & 1.06220 & 3.32990 & -0.99065 \\ \text { B } & 0.25622 & 3.07833 & 0.58066 \\ \text { H } & 0.01228 & 2.96698 & -3.37701 \\ \text { H } & -1.33161 & 4.41720 & -1.02622 \\ \text { H } & 1.74545 & 4.28650 & -1.21675 \\ \text { H } & 0.33799 & 3.84144 & 1.49755 \\ \text { C } & 3.06187 & -1.51218 & 2.42882 \\ \text { N } & 2.90730 & -0.82879 & 1.11103 \\ \text { C } & 3.26927 & -3.02453 & 2.23411 \\ \text { H } & 3.41091 & -3.52868 & 3.19664 \\ \text { H } & 2.40339 & -3.47322 & 1.73524 \\ \text { H } & 4.15746 & -3.20254 & 1.61791 \\ \text { C } & 1.78472 & -1.26113 & 3.23454 \\ \text { H } & 1.65512 & -0.19162 & 3.42721 \\ \text { H } & 0.90830 & -1.62929 & 2.69277 \\ \text { H } & 1.83965 & -1.78148 & 4.19678 \\ \text { C } & 4.23866 & -0.95950 & 3.24404 \\ \text { H } & 4.22999 & -1.41809 & 4.23945 \\ \text { H } & 5.20129 & -1.17866 & 2.77918 \\ \text { H } & 4.15442 & 0.12434 & 3.36688\end{array}$

\section{Calculated cartesian coordinates and energies of TS(B-B')}

$\mathrm{E}($ UB3PW91) $=$ $-3188.757082 E_{h}$ Sum of electronic and zero-point Energies $=\quad-3187.887190 \mathrm{E}_{\mathrm{h}}$ Sum of electronic and thermal Energies $=\quad-3187.836308 E_{h}$ Sum of electronic and thermal Enthalpies $=\quad-3187.835364 E_{h}$ Sum of electronic and thermal Free Energies $=-3187.969214 \mathrm{E}_{\mathrm{h}}$ Number of imaginary frequencies: $1\left(v=-911.5 \mathrm{~cm}^{-1}\right)$

$\begin{array}{lrrr}\text { K } & -0.35924 & 6.53422 & -0.24342 \\ \mathrm{Si} & -1.43658 & -1.15496 & 0.00167 \\ \mathrm{Si} & 1.63215 & -1.07627 & 0.01618 \\ \mathrm{Si} & 0.13616 & -2.72157 & 0.35911 \\ \mathrm{C} & -3.34702 & -1.92518 & -2.30578 \\ \mathrm{C} & -3.11103 & -0.83920 & 2.50047 \\ \mathrm{C} & 2.38356 & -1.62149 & -3.20932 \\ \mathrm{H} & 2.14644 & -0.57134 & -3.38706 \\ \mathrm{H} & 2.61216 & -2.08677 & -4.17313 \\ \mathrm{H} & 1.50326 & -2.11628 & -2.78655 \\ \mathrm{C} & -5.20212 & -0.90961 & 0.13038 \\ \mathrm{C} & -7.14382 & 0.54326 & 0.33486 \\ \mathrm{H} & -7.55791 & 1.54070 & 0.45776 \\ \mathrm{C} & -1.83003 & -0.44866 & 3.23974\end{array}$




\begin{tabular}{|c|c|c|c|}
\hline $\mathrm{H}$ & -1.62904 & 0.61977 & 3.12596 \\
\hline $\mathrm{H}$ & -1.93673 & -0.66926 & 4.30649 \\
\hline $\mathrm{H}$ & -0.97485 & -1.01002 & 2.85335 \\
\hline$c$ & -4.51764 & -1.12357 & -2.89369 \\
\hline $\mathrm{H}$ & -4.31962 & -0.05022 & -2.81671 \\
\hline $\mathrm{H}$ & -4.62817 & -1.37861 & -3.95269 \\
\hline $\mathrm{H}$ & -5.46419 & -1.33549 & -2.39548 \\
\hline C & 4.73580 & -0.91209 & -2.85626 \\
\hline $\mathrm{H}$ & 5.68784 & -1.09120 & -2.35559 \\
\hline $\mathrm{H}$ & 4.85918 & -1.15952 & -3.91565 \\
\hline $\mathrm{H}$ & 4.49912 & 0.15329 & -2.77694 \\
\hline C & 3.59155 & -1.75718 & -2.27688 \\
\hline C & -6.07169 & -2.01207 & 0.00150 \\
\hline $\mathrm{H}$ & -5.65838 & -3.01059 & -0.08565 \\
\hline C & 5.38047 & -0.65269 & 0.17051 \\
\hline C & -4.24644 & 0.04191 & 3.04003 \\
\hline $\mathrm{H}$ & -5.22802 & -0.27996 & 2.69044 \\
\hline $\mathrm{H}$ & -4.24659 & -0.00695 & 4.13429 \\
\hline $\mathrm{H}$ & -4.09600 & 1.08445 & 2.74251 \\
\hline C & -3.68541 & -3.42430 & -2.24973 \\
\hline $\mathrm{H}$ & -4.55488 & -3.61369 & -1.61845 \\
\hline $\mathrm{H}$ & -3.90994 & -3.80102 & -3.25353 \\
\hline $\mathrm{H}$ & -2.83648 & -3.98630 & -1.84813 \\
\hline C & -2.13703 & -1.74602 & -3.22839 \\
\hline $\mathrm{H}$ & -1.24445 & -2.21532 & -2.80240 \\
\hline $\mathrm{H}$ & -2.34421 & -2.21324 & -4.19614 \\
\hline $\mathrm{H}$ & -1.93140 & -0.68824 & -3.39965 \\
\hline C & -3.42554 & -2.32021 & 2.76648 \\
\hline $\mathrm{H}$ & -2.59798 & -2.94801 & 2.41902 \\
\hline $\mathrm{H}$ & -3.57440 & -2.49897 & 3.83690 \\
\hline $\mathrm{H}$ & -4.33776 & -2.62067 & 2.24112 \\
\hline C & -5.76682 & 0.37215 & 0.30836 \\
\hline $\mathrm{H}$ & -5.10276 & 1.22527 & 0.40970 \\
\hline C & 3.98378 & -3.24278 & -2.21727 \\
\hline $\mathrm{H}$ & 3.14825 & -3.83512 & -1.83149 \\
\hline $\mathrm{H}$ & 4.24017 & -3.60972 & -3.21708 \\
\hline $\mathrm{H}$ & 4.84747 & -3.40110 & -1.56949 \\
\hline C & 5.88298 & 0.65365 & 0.35732 \\
\hline $\mathrm{H}$ & 5.17911 & 1.47435 & 0.45796 \\
\hline C & 8.15727 & -0.16831 & 0.24940 \\
\hline $\mathrm{H}$ & 9.22679 & 0.01816 & 0.27613 \\
\hline C & 6.30248 & -1.71173 & 0.03953 \\
\hline $\mathrm{H}$ & 5.93755 & -2.72816 & -0.05700 \\
\hline C & -7.99874 & -0.55760 & 0.19359 \\
\hline $\mathrm{H}$ & -9.07603 & -0.42209 & 0.21430 \\
\hline C & 7.25017 & 0.88987 & 0.39124 \\
\hline $\mathrm{H}$ & 7.61564 & 1.90541 & 0.52000 \\
\hline B & 1.47197 & 1.72567 & -1.72194 \\
\hline $\mathrm{H}$ & 2.49626 & 1.46869 & -2.26470 \\
\hline C & -7.45027 & -1.83370 & 0.03244 \\
\hline $\mathrm{H}$ & -8.10248 & -2.69855 & -0.05655 \\
\hline
\end{tabular}




$\begin{array}{llll}\text { C } & 7.67076 & -1.46817 & 0.07862 \\ \text { H } & 8.36380 & -2.30047 & -0.01221 \\ \text { B } & 0.06163 & 0.69996 & -2.01816 \\ \text { H } & 0.09057 & -0.25145 & -2.70722 \\ \text { N } & 3.18698 & -1.30982 & -0.93341 \\ \text { N } & -2.97162 & -1.46096 & -0.95920 \\ \text { N } & -2.86937 & -0.63258 & 1.05044 \\ \text { C } & -3.75100 & -1.03067 & 0.07182 \\ \text { C } & 3.93773 & -0.84264 & 0.10264 \\ \text { B } & 0.03196 & 1.43611 & 0.71820 \\ \text { H } & 0.04404 & 0.96276 & 1.79492 \\ \text { B } & -1.42277 & 2.10963 & -0.02952 \\ \text { H } & -2.43333 & 2.10678 & 0.59210 \\ \text { B } & 1.45290 & 2.18823 & -0.02025 \\ \text { H } & 2.45734 & 2.23964 & 0.60867 \\ \text { C } & 0.88445 & 0.66140 & -0.53268 \\ \text { C } & -0.76905 & 0.61714 & -0.53897 \\ \text { B } & -1.40433 & 1.64844 & -1.73246 \\ \text { H } & -2.40949 & 1.33594 & -2.28204 \\ \text { B } & 0.01792 & 2.39749 & -2.50625 \\ \text { B } & -0.90011 & 3.28058 & -1.25880 \\ \text { B } & 0.87820 & 3.33041 & -1.25287 \\ \text { B } & -0.01276 & 3.14566 & 0.28085 \\ \text { H } & 0.01295 & 2.70149 & -3.65889 \\ \text { H } & -1.57796 & 4.22968 & -1.52951 \\ \text { H } & 1.50193 & 4.31685 & -1.51660 \\ \text { H } & -0.03576 & 3.98115 & 1.13674 \\ \text { C } & 3.27285 & -0.68590 & 2.52663 \\ \text { N } & 3.03093 & -0.48728 & 1.07544 \\ \text { C } & 3.66500 & -2.14872 & 2.79091 \\ \text { H } & 3.81072 & -2.32348 & 3.86244 \\ \text { H } & 2.87754 & -2.81996 & 2.43145 \\ \text { H } & 4.59872 & -2.39652 & 2.27596 \\ \text { C } & 1.96735 & -0.36756 & 3.25753 \\ \text { H } & 1.70843 & 0.68835 & 3.14372 \\ \text { H } & 1.14739 & -0.97459 & 2.86405 \\ \text { H } & 2.07836 & -0.58359 & 4.32471 \\ \text { C } & 4.35536 & 0.25318 & 3.07724 \\ \text { H } & 4.35105 & 0.20000 & 4.17131 \\ \text { H } & 5.35530 & -0.01383 & 2.73332 \\ \text { H } & 4.15089 & 1.28728 & 2.78256\end{array}$

Calculated cartesian coordinates and energies of TS(B-C)

$\mathrm{E}($ UB3PW91) $=$ $-3188.756679 E_{h}$ Sum of electronic and zero-point Energies $=\quad-3187.886973 \mathrm{E}_{\mathrm{h}}$ Sum of electronic and thermal Energies $=\quad-3187.835597 \mathrm{E}_{\mathrm{h}}$ Sum of electronic and thermal Enthalpies $=\quad-3187.834652 \mathrm{E}_{\mathrm{h}}$ Sum of electronic and thermal Free Energies $=\quad-3187.970167 \mathrm{E}_{\mathrm{h}}$ Number of imaginary frequencies: $1\left(v=-1040.6 \mathrm{~cm}^{-1}\right)$ 


\begin{tabular}{|c|c|c|c|}
\hline K & 0.38583 & 6.45934 & -0.15340 \\
\hline$S$ & -1.50570 & -1.00600 & -0.02122 \\
\hline & 1.63130 & -1.10564 & -0.00573 \\
\hline$S$ & 0.02717 & -2.64845 & 0.29934 \\
\hline$C$ & -3.47328 & -1.75739 & -2.32845 \\
\hline 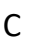 & -3.30616 & -0.46739 & 2.49071 \\
\hline$C$ & 2.27457 & -1.69290 & -3.16870 \\
\hline 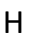 & 2.04860 & -0.63305 & -3.29741 \\
\hline $\mathrm{H}$ & 2.45924 & -2.12406 & -4.15779 \\
\hline $\mathrm{H}$ & 1.39890 & -2.18614 & -2.73424 \\
\hline C & -5.31549 & -0.86875 & 0.08474 \\
\hline C & -7.35044 & 0.40262 & -0.18971 \\
\hline $\mathrm{H}$ & -7.84930 & 1.33922 & -0.42037 \\
\hline 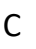 & -2.02951 & 0.05187 & 3.15520 \\
\hline $\mathrm{H}$ & -1.78608 & 1.05374 & 2.79505 \\
\hline $\mathrm{H}$ & -2.17059 & 0.09695 & 4.23914 \\
\hline $\mathrm{H}$ & -1.18531 & -0.61034 & 2.94122 \\
\hline 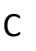 & -4.69104 & -1.05425 & -2.94226 \\
\hline$\pi$ & -4.54582 & 0.03034 & -2.94752 \\
\hline $\mathrm{H}$ & -4.80923 & -1.39097 & -3.97698 \\
\hline $\mathrm{H}$ & -5.61662 & -1.28011 & -2.41063 \\
\hline 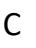 & 4.64808 & -1.03327 & -2.87435 \\
\hline 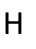 & 5.61333 & -1.23636 & -2.40977 \\
\hline $\mathrm{H}$ & 4.73606 & -1.24459 & -3.94528 \\
\hline $\mathrm{H}$ & 4.42485 & 0.03058 & -2.75023 \\
\hline C & 3.51309 & -1.88461 & -2.28519 \\
\hline 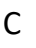 & -6.06369 & -2.00502 & 0.40210 \\
\hline $\mathrm{H}$ & -5.56092 & -2.93945 & 0.63017 \\
\hline C & 5.36790 & -0.83992 & 0.19409 \\
\hline C & -4.43308 & 0.54441 & 2.73819 \\
\hline 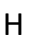 & -5.40987 & 0.16190 & 2.43795 \\
\hline$\Pi$ & -4.47742 & 0.77381 & 3.80759 \\
\hline $\mathrm{H}$ & -4.23961 & 1.47349 & 2.19336 \\
\hline C & -3.75448 & -3.25517 & -2.13725 \\
\hline $\mathrm{H}$ & -4.60978 & -3.41077 & -1.47423 \\
\hline $\mathrm{H}$ & -3.98218 & -3.72360 & -3.10046 \\
\hline $\mathrm{H}$ & -2.87922 & -3.75038 & -1.70493 \\
\hline 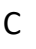 & -2.28529 & -1.58514 & -3.27751 \\
\hline $\mathrm{H}$ & -1.38267 & -2.03422 & -2.85232 \\
\hline $\mathrm{H}$ & -2.50397 & -2.07710 & -4.23001 \\
\hline $\mathrm{H}$ & -2.09325 & -0.52682 & -3.46937 \\
\hline C & -3.66779 & -1.83359 & 3.09190 \\
\hline $\mathrm{H}$ & -2.86341 & -2.55197 & 2.90744 \\
\hline $\mathrm{H}$ & -3.81546 & -1.74327 & 4.17316 \\
\hline $\mathrm{H}$ & -4.59186 & -2.22221 & 2.65640 \\
\hline C & -5.96101 & 0.33713 & -0.21192 \\
\hline $\mathrm{H}$ & -5.36968 & 1.21381 & -0.45737 \\
\hline C & 3.88008 & -3.37836 & -2.29661 \\
\hline $\mathrm{H}$ & 3.03717 & -3.97107 & -1.92679 \\
\hline 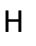 & 4.12088 & -3.70773 & -3.31331 \\
\hline $\mathrm{H}$ & 4.74423 & -3.58311 & -1.66161 \\
\hline C & 5.81995 & 0.43903 & 0.60508 \\
\hline $\mathrm{H}$ & 5.07896 & 1.19269 & 0.85281 \\
\hline C & 8.13132 & -0.23092 & 0.33012 \\
\hline $\mathrm{H}$ & 9.19098 & 0.00148 & 0.38167 \\
\hline$c$ & 6.34747 & -1.80439 & -0.13792 \\
\hline
\end{tabular}




$\begin{array}{lrrr}\text { H } & 6.03576 & -2.80477 & -0.41639 \\ \text { C } & -8.10003 & -0.73253 & 0.12612 \\ \text { H } & -9.18468 & -0.68007 & 0.14148 \\ \text { C } & 7.17338 & 0.73446 & 0.66851 \\ \text { H } & 7.48965 & 1.72974 & 0.97189 \\ \text { B } & 1.54621 & 1.71761 & -1.73495 \\ \text { H } & 2.49801 & 1.44643 & -2.39188 \\ \text { C } & -7.45609 & -1.93342 & 0.42176 \\ \text { H } & -8.03563 & -2.81815 & 0.66774 \\ \text { C } & 7.70158 & -1.50096 & -0.07116 \\ \text { H } & 8.43149 & -2.26686 & -0.32165 \\ \text { B } & 0.03724 & 0.75293 & -1.89090 \\ \text { H } & -0.02596 & -0.15555 & -2.64112 \\ \text { N } & 3.15755 & -1.49308 & -0.91463 \\ \text { N } & -3.08332 & -1.17483 & -1.02972 \\ \text { N } & -3.00934 & -0.63363 & 1.05593 \\ \text { C } & -3.83456 & -0.90879 & 0.04351 \\ \text { C } & 3.94433 & -1.09638 & 0.14353 \\ \text { B } & 0.16193 & 1.39572 & 0.68929 \\ \text { H } & 0.19361 & 0.94694 & 1.77958 \\ \text { B } & -1.30059 & 2.26195 & 0.08197 \\ \text { H } & -2.25973 & 2.37716 & 0.77837 \\ \text { B } & 1.62960 & 2.15095 & -0.02653 \\ \text { H } & 2.63553 & 2.19356 & 0.60316 \\ \text { C } & 1.08297 & 0.66326 & -0.54027 \\ \text { C } & -0.90408 & 0.75465 & -0.47581 \\ \text { B } & -1.38247 & 1.83362 & -1.63391 \\ \text { H } & -2.40178 & 1.63132 & -2.21442 \\ \text { B } & 0.08315 & 2.46300 & -2.43181 \\ \text { B } & -0.72258 & 3.40978 & -1.14813 \\ \text { B } & 1.04865 & 3.34002 & -1.21220 \\ \text { B } & 0.21077 & 3.15544 & 0.35528 \\ \text { H } & 0.06156 & 2.77854 & -3.58219 \\ \text { H } & -1.32606 & 4.41981 & -1.37628 \\ \text { H } & 1.71053 & 4.29967 & -1.48961 \\ \text { H } & 0.27789 & 3.96618 & 1.23434 \\ \text { C } & 3.17361 & -1.07575 & 2.54449 \\ \text { N } & 3.02531 & -0.69850 & 1.11415 \\ \text { C } & 3.32591 & -2.60113 & 2.66721 \\ \text { H } & 3.43377 & -2.89937 & 3.71593 \\ \text { H } & 2.44654 & -3.10590 & 2.25258 \\ \text { H } & 4.21292 & -2.93860 & 2.11989 \\ \text { C } & 1.90854 & -0.62489 & 3.27824 \\ \text { H } & 1.80334 & 0.46290 & 3.23362 \\ \text { H } & 1.02058 & -1.08284 & 2.83223 \\ \text { H } & 1.96141 & -0.92605 & 4.32958 \\ \text { C } & 4.37122 & -0.39356 & 3.21923 \\ \text { H } & 4.34177 & -0.60485 & 4.29388 \\ \text { H } & 5.32642 & -0.75225 & 2.83258 \\ \text { H } & 4.32827 & 0.69103 & 3.08263\end{array}$

Calculated cartesian coordinates and energies of $C$ $\mathrm{E}($ UB3PW91) $=$ $-3188.787143 E_{h}$ Sum of electronic and zero-point Energies $=\quad-3187.914571 \mathrm{E}_{\mathrm{h}}$ Sum of electronic and thermal Energies $=\quad-3187.863055 \mathrm{E}_{\mathrm{h}}$ 
$\begin{array}{ll}\text { Sum of electronic and thermal Enthalpies }= & -3187.862111 E_{h} \\ \text { Sum of electronic and thermal Free Energies }= & -3187.996636 E_{h} \\ \text { Number of imaginary frequencies: } 0 & \end{array}$

\begin{tabular}{llll}
$\mathrm{K}$ & 0.02959 & 6.59552 & -0.01218 \\
$\mathrm{Si}$ & -1.52633 & -0.81237 & -0.05109 \\
$\mathrm{Si}$ & 1.66275 & -0.79226 & -0.07446 \\
$\mathrm{Si}$ & 0.08027 & -2.38108 & 0.26920 \\
$\mathrm{C}$ & -3.42017 & -1.65833 & -2.39814 \\
$\mathrm{C}$ & -3.28433 & -0.71766 & 2.51435 \\
$\mathrm{C}$ & 2.30002 & -1.34396 & -3.31913 \\
$\mathrm{H}$ & 2.09163 & -0.27287 & -3.37070 \\
$\mathrm{H}$ & 2.47804 & -1.71303 & -4.33372 \\
$\mathrm{H}$ & 1.41811 & -1.84888 & -2.91267 \\
$\mathrm{C}$ & -5.29295 & -1.35233 & 0.14629 \\
$\mathrm{C}$ & -7.54297 & -0.47648 & 0.06697 \\
$\mathrm{H}$ & -8.22101 & 0.35912 & -0.07906 \\
$\mathrm{C}$ & -2.12942 & 0.06752 & 3.14195 \\
$\mathrm{H}$ & -2.11184 & 1.09466 & 2.76802 \\
$\mathrm{H}$ & -2.24400 & 0.08914 & 4.22997 \\
$\mathrm{H}$ & -1.17083 & -0.40192 & 2.90250 \\
$\mathrm{C}$ & -4.61693 & -0.88142 & -2.96284 \\
$\mathrm{H}$ & -4.44964 & 0.19574 & -2.86570 \\
$\mathrm{H}$ & -4.73374 & -1.11830 & -4.02523 \\
$\mathrm{H}$ & -5.55012 & -1.13785 & -2.45837 \\
$\mathrm{C}$ & 4.71765 & -0.82856 & -3.02874 \\
$\mathrm{H}$ & 5.65872 & -1.08135 & -2.53733 \\
$\mathrm{H}$ & 4.82173 & -1.06197 & -4.09321 \\
$\mathrm{H}$ & 4.54607 & 0.24748 & -2.92662 \\
$\mathrm{C}$ & 3.53202 & -1.61315 & -2.45106 \\
$\mathrm{C}$ & -5.79554 & -2.62245 & 0.44131 \\
$\mathrm{H}$ & -5.11280 & -3.45404 & 0.58539 \\
$\mathrm{C}$ & 5.43719 & -1.29212 & 0.06678 \\
$\mathrm{C}$ & -4.60303 & -0.01800 & 2.86917 \\
$\mathrm{H}$ & -5.47683 & -0.60549 & 2.58266 \\
$\mathrm{H}$ & -5.167010 & -0.70543 & -0.26820 \\
$\mathrm{H}$ & -4.64304 & 0.13442 & 3.95241 \\
$\mathrm{H}$ & -4.66453 & 0.95937 & 2.38074 \\
$\mathrm{C}$ & -3.70999 & -3.16631 & -2.38502 \\
$\mathrm{H}$ & -4.60326 & -3.39175 & -1.79716 \\
$\mathrm{H}$ & -3.87730 & -3.52608 & -3.40563 \\
$\mathrm{H}$ & -2.86191 & -3.71046 & -1.95765 \\
$\mathrm{C}$ & -2.19953 & -1.38571 & -3.28092 \\
$\mathrm{H}$ & -1.31144 & -1.88686 & -2.88289 \\
$\mathrm{H}$ & -2.38763 & -1.75717 & -4.29281 \\
$\mathrm{H}$ & -1.99585 & -0.31392 & -3.33635 \\
$\mathrm{H}$ & -3.27702 & -2.15588 & 3.05300 \\
$\mathrm{H}$ & -3.3813 & -2.64875 & 2.78850 \\
\hline & -2.15462 & 4.14321 \\
$\mathrm{H}$ & & & \\
$\mathrm{H}$ & -10543 & -2.73291 & 2.63257 \\
\hline
\end{tabular}




\begin{tabular}{|c|c|c|c|}
\hline$C$ & 3.83054 & -3.11959 & -2.44540 \\
\hline$H$ & 2.99104 & -3.67092 & -2.01020 \\
\hline$H$ & 3.98895 & -3.47539 & -3.46883 \\
\hline $\mathrm{H}$ & 4.73182 & -3.34077 & -1.86819 \\
\hline C & 6.30142 & -0.20929 & -0.13070 \\
\hline $\mathrm{H}$ & 5.88954 & 0.77104 & -0.34905 \\
\hline C & 8.19637 & -1.65953 & 0.24510 \\
\hline $\mathrm{H}$ & 9.27071 & -1.80242 & 0.31544 \\
\hline C & 5.95547 & -2.55829 & 0.35135 \\
\hline $\mathrm{H}$ & 5.28235 & -3.39638 & 0.50290 \\
\hline C & -8.04590 & -1.74454 & 0.36489 \\
\hline $\mathrm{H}$ & -9.11776 & -1.89709 & 0.45097 \\
\hline C & 7.67768 & -0.39543 & -0.04230 \\
\hline $\mathrm{H}$ & 8.34587 & 0.44674 & -0.19583 \\
\hline B & 1.54330 & 2.07393 & -1.63962 \\
\hline $\mathrm{H}$ & 2.51046 & 1.91977 & -2.32463 \\
\hline C & -7.17219 & -2.81527 & 0.55035 \\
\hline $\mathrm{H}$ & -7.55960 & -3.80325 & 0.78056 \\
\hline C & 7.33526 & -2.73873 & 0.44022 \\
\hline $\mathrm{H}$ & 7.73502 & -3.72365 & 0.66225 \\
\hline B & 0.04485 & 1.00193 & -1.71478 \\
\hline $\mathrm{H}$ & 0.04516 & 0.11824 & -2.50610 \\
\hline$N$ & 3.19978 & -1.17040 & -1.08572 \\
\hline$N$ & -3.07350 & -1.21019 & -1.03828 \\
\hline$N$ & -3.03507 & -0.74491 & 1.06337 \\
\hline C & -3.83136 & -1.11778 & 0.05692 \\
\hline C & 3.97232 & -1.07180 & -0.00136 \\
\hline B & 0.05902 & 1.54942 & 0.65515 \\
\hline $\mathrm{H}$ & 0.07079 & 1.11305 & 1.75803 \\
\hline B & -1.45849 & 2.45807 & 0.11452 \\
\hline $\mathrm{H}$ & -2.42293 & 2.61418 & 0.80363 \\
\hline B & 1.55549 & 2.47868 & 0.09175 \\
\hline $\mathrm{H}$ & 2.52777 & 2.64826 & 0.76644 \\
\hline C & 1.22417 & 0.98268 & -0.46710 \\
\hline C & -1.11520 & 0.96710 & -0.45016 \\
\hline B & -1.46711 & 2.05366 & -1.61728 \\
\hline $\mathrm{H}$ & -2.44218 & 1.88588 & -2.28781 \\
\hline B & 0.02839 & 2.70929 & -2.33523 \\
\hline B & -0.85636 & 3.62699 & -1.07829 \\
\hline B & 0.91905 & 3.63876 & -1.09177 \\
\hline B & 0.04496 & 3.35335 & 0.44724 \\
\hline $\mathrm{H}$ & 0.01780 & 3.02754 & -3.48824 \\
\hline $\mathrm{H}$ & -1.47693 & 4.62929 & -1.31349 \\
\hline $\mathrm{H}$ & 1.52260 & 4.64910 & -1.33675 \\
\hline $\mathrm{H}$ & 0.04653 & 4.14002 & 1.35553 \\
\hline C & 3.45660 & -0.68098 & 2.46477 \\
\hline$N$ & 3.18681 & -0.70920 & 1.01742 \\
\hline C & 3.46936 & -2.11952 & 3.00251 \\
\hline $\mathrm{H}$ & 3.59116 & -2.11777 & 4.09092 \\
\hline $\mathrm{H}$ & 2.52816 & -2.61987 & 2.75329 \\
\hline $\mathrm{H}$ & 4.29546 & -2.68993 & 2.56865 \\
\hline
\end{tabular}




$\begin{array}{lrrr}\mathrm{C} & 2.30387 & 0.09336 & 3.10968 \\ \mathrm{H} & 2.27179 & 1.12075 & 2.73741 \\ \mathrm{H} & 1.34626 & -0.38452 & 2.88358 \\ \mathrm{H} & 2.43382 & 0.11467 & 4.19598 \\ \mathrm{C} & 4.77388 & 0.03014 & 2.80146 \\ \mathrm{H} & 4.82784 & 0.18221 & 3.88414 \\ \mathrm{H} & 5.64868 & -0.54940 & 2.50221 \\ \mathrm{H} & 4.81981 & 1.00831 & 2.31287\end{array}$

\section{Calculated cartesian coordinates and energies of TS(C-D)}

$\mathrm{E}($ UB3PW91) $=$ $-3188.777119 E_{h}$ Sum of electronic and zero-point Energies $=\quad-3187.908413 E_{h}$ Sum of electronic and thermal Energies $=\quad-3187.856265 \mathrm{E}_{\mathrm{h}}$ Sum of electronic and thermal Enthalpies $=\quad-3187.855321 \mathrm{E}_{\mathrm{h}}$ Sum of electronic and thermal Free Energies $=-3187.994154 \mathrm{E}_{\mathrm{h}}$ Number of imaginary frequencies: $1\left(v=-1939.7 \mathrm{~cm}^{-1}\right)$

$\begin{array}{llll}\text { K } & 0.01809 & 6.64695 & -0.16017 \\ \mathrm{Si} & -1.60016 & -0.63624 & -0.07868 \\ \mathrm{Si} & 1.72761 & -0.62840 & -0.03849 \\ \mathrm{Si} & 0.06267 & -2.19995 & 0.32119 \\ \mathrm{C} & -3.46798 & -1.59862 & -2.37527 \\ \mathrm{C} & -3.25544 & -0.65076 & 2.54123 \\ \mathrm{C} & 2.22015 & -1.49038 & -3.22297 \\ \mathrm{H} & 2.20273 & -0.40772 & -3.37499 \\ \mathrm{H} & 2.31092 & -1.98189 & -4.19606 \\ \mathrm{H} & 1.26914 & -1.79022 & -2.77073 \\ \mathrm{C} & -5.27111 & -1.54500 & 0.25248 \\ \mathrm{C} & -7.60686 & -0.92932 & 0.25594 \\ \mathrm{H} & -8.37885 & -0.17347 & 0.14689 \\ \mathrm{C} & -2.09365 & 0.15674 & 3.12473 \\ \mathrm{H} & -2.09636 & 1.17820 & 2.73436 \\ \mathrm{H} & -2.17891 & 0.19511 & 4.21478 \\ \mathrm{H} & -1.13528 & -0.30633 & 2.86770 \\ \mathrm{C} & -4.61515 & -0.72796 & -2.90400 \\ \mathrm{H} & -4.36911 & 0.33188 & -2.78679 \\ \mathrm{H} & -4.77118 & -0.93232 & -3.96804 \\ \mathrm{H} & -5.55196 & -0.93184 & -2.38111 \\ \mathrm{C} & 4.70167 & -1.45851 & -2.99921 \\ \mathrm{H} & 5.58141 & -1.83845 & -2.47711 \\ \mathrm{H} & 4.72582 & -1.84691 & -4.02220 \\ \mathrm{H} & 4.76459 & -0.36690 & -3.04290 \\ \mathrm{C} & 3.39305 & -1.89453 & -2.32685 \\ \mathrm{C} & -5.61803 & -2.86982 & 0.53278 \\ \mathrm{H} & -4.84040 & -3.62095 & 0.63203 \\ \mathrm{C} & 5.40800 & -1.55211 & 0.10710 \\ \mathrm{C} & -4.57527 & 0.03109 & 2.92600 \\ \mathrm{H} & -5.44527 & -0.56859 & 2.65308 \\ \mathrm{H} & -4.59823 & 0.18020 & 4.01008 \\ \mathrm{H} & -4.65835 & 1.00845 & 2.44093 \\ \mathrm{C} & -3.83953 & -3.08646 & -2.43523\end{array}$




\begin{tabular}{|c|c|c|c|}
\hline $\mathrm{H}$ & -4.75915 & -3.29096 & -1.88241 \\
\hline$H$ & -3.99701 & -3.38817 & -3.47574 \\
\hline H & -3.03365 & -3.69531 & -2.01382 \\
\hline C & -2.22171 & -1.35294 & -3.23068 \\
\hline $\mathrm{H}$ & -1.36590 & -1.91744 & -2.84560 \\
\hline $\mathrm{H}$ & -2.41216 & -1.66962 & -4.26038 \\
\hline $\mathrm{H}$ & -1.95840 & -0.29258 & -3.23714 \\
\hline$c$ & -3.20930 & -2.08360 & 3.09029 \\
\hline $\mathrm{H}$ & -2.27316 & -2.56847 & 2.79542 \\
\hline $\mathrm{H}$ & -3.27002 & -2.07150 & 4.18350 \\
\hline $\mathrm{H}$ & -4.04628 & -2.67557 & 2.71074 \\
\hline C & -6.26829 & -0.57339 & 0.11446 \\
\hline $\mathrm{H}$ & -5.98967 & 0.45409 & -0.09768 \\
\hline C & 3.37371 & -3.41457 & -2.11392 \\
\hline $\mathrm{H}$ & 2.44943 & -3.71048 & -1.60688 \\
\hline $\mathrm{H}$ & 3.42651 & -3.93151 & -3.07771 \\
\hline $\mathrm{H}$ & 4.22545 & -3.73732 & -1.50935 \\
\hline C & 6.39100 & -0.62210 & -0.24841 \\
\hline $\mathrm{H}$ & 6.09741 & 0.37491 & -0.56175 \\
\hline C & 8.10162 & -2.27171 & 0.18797 \\
\hline $\mathrm{H}$ & 9.15042 & -2.55213 & 0.21805 \\
\hline C & 5.77476 & -2.84086 & 0.50547 \\
\hline $\mathrm{H}$ & 5.00829 & -3.55634 & 0.78707 \\
\hline C & -7.95387 & -2.25128 & 0.54003 \\
\hline $\mathrm{H}$ & -8.99858 & -2.52602 & 0.65239 \\
\hline C & 7.73497 & -0.98327 & -0.20444 \\
\hline $\mathrm{H}$ & 8.49576 & -0.25916 & -0.48017 \\
\hline B & 1.63871 & 2.15553 & -1.65350 \\
\hline $\mathrm{H}$ & 2.62911 & 2.03326 & -2.31634 \\
\hline C & -6.95958 & -3.21950 & 0.67829 \\
\hline $\mathrm{H}$ & -7.22555 & -4.24914 & 0.89778 \\
\hline C & 7.12154 & -3.19862 & 0.54221 \\
\hline $\mathrm{H}$ & 7.40270 & -4.20142 & 0.84949 \\
\hline B & 0.08805 & 1.04307 & -1.66782 \\
\hline $\mathrm{H}$ & 0.10624 & 0.13702 & -2.44134 \\
\hline$N$ & 3.19018 & -1.20873 & -1.03752 \\
\hline$N$ & -3.11040 & -1.21833 & -0.99905 \\
\hline$N$ & -3.05806 & -0.66954 & 1.08077 \\
\hline C & -3.84617 & -1.15753 & 0.11299 \\
\hline C & 3.97746 & -1.16545 & 0.04530 \\
\hline B & 0.04012 & 1.62215 & 0.53232 \\
\hline $\mathrm{H}$ & 0.02130 & 1.22013 & 1.65479 \\
\hline B & -1.51201 & 2.58998 & -0.03841 \\
\hline $\mathrm{H}$ & -2.50086 & 2.82380 & 0.59680 \\
\hline B & 1.56220 & 2.62406 & 0.05845 \\
\hline $\mathrm{H}$ & 2.50834 & 2.84033 & 0.76110 \\
\hline C & 1.30411 & 1.09521 & -0.47381 \\
\hline C & -1.17609 & 1.08407 & -0.52799 \\
\hline B & -1.42888 & 2.14588 & -1.75644 \\
\hline $\mathrm{H}$ & -2.37912 & 1.98495 & -2.46727 \\
\hline B & 0.10927 & 2.72479 & -2.41276 \\
\hline
\end{tabular}




$\begin{array}{lrrr}\text { B } & -0.82361 & 3.70809 & -1.22140 \\ \text { B } & 0.95843 & 3.71458 & -1.18766 \\ \text { B } & 0.02337 & 3.45077 & 0.33713 \\ \text { H } & 0.14243 & 2.99340 & -3.58028 \\ \text { H } & -1.41301 & 4.71810 & -1.51078 \\ \text { H } & 1.54989 & 4.73063 & -1.45058 \\ \text { H } & -0.00553 & 4.25304 & 1.23433 \\ \text { C } & 3.59737 & -0.34696 & 2.43543 \\ \text { N } & 3.23641 & -0.69606 & 1.05216 \\ \text { C } & 4.01518 & -1.60260 & 3.21300 \\ \text { H } & 4.17325 & -1.35041 & 4.26662 \\ \text { H } & 3.23287 & -2.36529 & 3.15185 \\ \text { H } & 4.94549 & -2.02323 & 2.82502 \\ \text { C } & 2.33762 & 0.24655 & 3.07225 \\ \text { H } & 2.04263 & 1.16543 & 2.56002 \\ \text { H } & 1.50299 & -0.46071 & 3.01814 \\ \text { H } & 2.52905 & 0.47963 & 4.12380 \\ \text { C } & 4.71434 & 0.70478 & 2.46175 \\ \text { H } & 4.87284 & 1.04760 & 3.48934 \\ \text { H } & 5.65898 & 0.29945 & 2.09276 \\ \text { H } & 4.43711 & 1.56647 & 1.84693\end{array}$

\section{Calculated cartesian coordinates and energies of $D$}

$\begin{array}{ll}\text { E(UB3PW91) = } & -3188.783521 \mathrm{E}_{\mathrm{h}} \\ \text { Sum of electronic and zero-point Energies }= & -3187.914021 \mathrm{E}_{\mathrm{h}} \\ \text { Sum of electronic and thermal Energies= } & -3187.861261 \mathrm{E}_{\mathrm{h}} \\ \text { Sum of electronic and thermal Enthalpies }= & -3187.860317 \mathrm{E}_{\mathrm{h}} \\ \text { Sum of electronic and thermal Free Energies= } & -3188.001002 \mathrm{E}_{\mathrm{h}} \\ \text { Number of imaginary frequencies: } 0 & \end{array}$

$\begin{array}{lrrr}\text { K } & 0.02672 & 6.61958 & -0.48506 \\ \mathrm{Si} & -1.62974 & -0.55034 & 0.05278 \\ \mathrm{Si} & 1.80206 & -0.51475 & -0.00211 \\ \mathrm{Si} & 0.11201 & -2.02434 & 0.66951 \\ \mathrm{C} & -3.27992 & -1.95070 & -2.18064 \\ \mathrm{C} & -3.45951 & -0.20396 & 2.52938 \\ \mathrm{C} & 2.10310 & -1.72815 & -3.05405 \\ \mathrm{H} & 2.07753 & -0.65682 & -3.27195 \\ \mathrm{H} & 2.14394 & -2.27923 & -3.99797 \\ \mathrm{H} & 1.17225 & -1.99595 & -2.54353 \\ \mathrm{C} & -5.22593 & -1.70097 & 0.33084 \\ \mathrm{C} & -7.57973 & -1.31480 & -0.04900 \\ \mathrm{H} & -8.38002 & -0.67453 & -0.40758 \\ \mathrm{C} & -2.23320 & 0.52769 & 3.08204 \\ \mathrm{H} & -2.00353 & 1.41037 & 2.47945 \\ \mathrm{H} & -2.42391 & 0.84705 & 4.11074 \\ \mathrm{H} & -1.35525 & -0.12766 & 3.08189 \\ \mathrm{C} & -4.09159 & -0.95081 & -3.01504 \\ \mathrm{H} & -3.57530 & 0.01221 & -3.05858 \\ \mathrm{H} & -4.22031 & -1.32867 & -4.03452 \\ \mathrm{H} & -5.08377 & -0.79758 & -2.58216\end{array}$




\begin{tabular}{|c|c|c|c|}
\hline C & 4.59512 & -1.70649 & -2.95609 \\
\hline$H$ & 5.49598 & -2.03183 & -2.43272 \\
\hline $\mathrm{H}$ & 4.58385 & -2.18980 & -3.93792 \\
\hline $\mathrm{H}$ & 4.64718 & -0.62365 & -3.10370 \\
\hline C & 3.31834 & -2.08070 & -2.19278 \\
\hline C & -5.51764 & -2.95426 & 0.87878 \\
\hline $\mathrm{H}$ & -4.70965 & -3.59110 & 1.22526 \\
\hline C & 5.39325 & -1.69500 & 0.19735 \\
\hline C & -4.64952 & 0.76436 & 2.49733 \\
\hline $\mathrm{H}$ & -5.56465 & 0.26732 & 2.16764 \\
\hline $\mathrm{H}$ & -4.82555 & 1.16566 & 3.50040 \\
\hline $\mathrm{H}$ & -4.43889 & 1.59821 & 1.82061 \\
\hline C & -4.00818 & -3.29892 & -2.09894 \\
\hline $\mathrm{H}$ & -5.04267 & -3.19257 & -1.76812 \\
\hline $\mathrm{H}$ & -4.02128 & -3.75712 & -3.09268 \\
\hline $\mathrm{H}$ & -3.48945 & -3.97672 & -1.41410 \\
\hline C & -1.91192 & -2.16887 & -2.83372 \\
\hline $\mathrm{H}$ & -1.31147 & -2.87961 & -2.25619 \\
\hline $\mathrm{H}$ & -2.04314 & -2.56551 & -3.84453 \\
\hline $\mathrm{H}$ & -1.35798 & -1.22878 & -2.90558 \\
\hline C & -3.76784 & -1.41913 & 3.41344 \\
\hline $\mathrm{H}$ & -2.93598 & -2.12966 & 3.38699 \\
\hline $\mathrm{H}$ & -3.91529 & -1.09566 & 4.44878 \\
\hline $\mathrm{H}$ & -4.67648 & -1.93027 & 3.08817 \\
\hline C & -6.25830 & -0.87996 & -0.13269 \\
\hline $\mathrm{H}$ & -6.02553 & 0.09727 & -0.54419 \\
\hline C & 3.29762 & -3.58049 & -1.86796 \\
\hline $\mathrm{H}$ & 2.40092 & -3.82730 & -1.29045 \\
\hline $\mathrm{H}$ & 3.29029 & -4.16483 & -2.79370 \\
\hline $\mathrm{H}$ & 4.17830 & -3.87251 & -1.29015 \\
\hline C & 6.44254 & -0.89329 & -0.26426 \\
\hline $\mathrm{H}$ & 6.22666 & 0.08424 & -0.68405 \\
\hline C & 8.02001 & -2.61861 & 0.34526 \\
\hline $\mathrm{H}$ & 9.04306 & -2.97863 & 0.40232 \\
\hline C & 5.65948 & -2.95827 & 0.73400 \\
\hline $\mathrm{H}$ & 4.84087 & -3.57284 & 1.09589 \\
\hline C & -7.87122 & -2.56624 & 0.49382 \\
\hline $\mathrm{H}$ & -8.90144 & -2.90426 & 0.55592 \\
\hline C & 7.75380 & -1.35627 & -0.18685 \\
\hline $\mathrm{H}$ & 8.56660 & -0.73150 & -0.54463 \\
\hline B & 1.57854 & 2.11624 & -1.84032 \\
\hline $\mathrm{H}$ & 2.54232 & 1.95546 & -2.53680 \\
\hline C & -6.83992 & -3.38413 & 0.95770 \\
\hline $\mathrm{H}$ & -7.06383 & -4.35947 & 1.37917 \\
\hline C & 6.97321 & -3.41824 & 0.80406 \\
\hline $\mathrm{H}$ & 7.17705 & -4.40075 & 1.21893 \\
\hline B & 0.03485 & 0.97409 & -1.62049 \\
\hline $\mathrm{H}$ & 0.03223 & 0.00419 & -2.32327 \\
\hline $\mathrm{N}$ & 3.18709 & -1.29276 & -0.95509 \\
\hline $\mathrm{N}$ & -3.02357 & -1.37779 & -0.84782 \\
\hline $\mathrm{N}$ & -3.10415 & -0.62704 & 1.16578 \\
\hline
\end{tabular}




$\begin{array}{lrrr}\text { C } & -3.81885 & -1.24717 & 0.22052 \\ \text { C } & 3.99736 & -1.20219 & 0.10872 \\ \text { B } & 0.07200 & 1.66337 & 0.41802 \\ \text { H } & 0.09201 & 1.32146 & 1.56897 \\ \text { B } & -1.50145 & 2.66821 & -0.10088 \\ \text { H } & -2.46520 & 2.95385 & 0.55558 \\ \text { B } & 1.62606 & 2.69627 & -0.16998 \\ \text { H } & 2.62041 & 3.00462 & 0.42805 \\ \text { C } & 1.33589 & 1.13486 & -0.54933 \\ \text { C } & -1.22457 & 1.10983 & -0.51750 \\ \text { B } & -1.53929 & 2.10645 & -1.77696 \\ \text { H } & -2.53627 & 1.96480 & -2.42790 \\ \text { B } & 0.00459 & 2.58466 & -2.53857 \\ \text { B } & -0.86866 & 3.67276 & -1.38940 \\ \text { B } & 0.91725 & 3.68317 & -1.42900 \\ \text { B } & 0.06049 & 3.49507 & 0.15548 \\ \text { H } & -0.01696 & 2.76503 & -3.72563 \\ \text { H } & -1.44899 & 4.67588 & -1.72737 \\ \text { H } & 1.46972 & 4.69298 & -1.79265 \\ \text { H } & 0.06689 & 4.34933 & 1.00712 \\ \text { C } & 3.72044 & -0.09442 & 2.39747 \\ \text { N } & 3.30869 & -0.58069 & 1.07086 \\ \text { C } & 4.11367 & -1.26774 & 3.30381 \\ \text { H } & 4.30363 & -0.90318 & 4.31828 \\ \text { H } & 3.30410 & -2.00303 & 3.34349 \\ \text { H } & 5.01973 & -1.76305 & 2.94836 \\ \text { C } & 2.50106 & 0.61809 & 2.98872 \\ \text { H } & 2.21234 & 1.47157 & 2.36972 \\ \text { H } & 1.64577 & -0.06305 & 3.05737 \\ \text { H } & 2.73421 & 0.97976 & 3.99438 \\ \text { C } & 4.87439 & 0.90836 & 2.26683 \\ \text { H } & 5.08883 & 1.35444 & 3.24313 \\ \text { H } & 5.78687 & 0.42590 & 1.90920 \\ \text { H } & 4.60176 & 1.70760 & 1.57098\end{array}$

\section{Calculated cartesian coordinates and energies of TS(D-E)}

$\mathrm{E}(\mathrm{UB} 3 \mathrm{PW} 91)=$ $-3188.779858 E_{h}$ Sum of electronic and zero-point Energies $=\quad-3187.910543 E_{h}$ Sum of electronic and thermal Energies= $\quad-3187.858400 \mathrm{E}_{\mathrm{h}}$ Sum of electronic and thermal Enthalpies $=\quad-3187.857456 \mathrm{E}_{\mathrm{h}}$ Sum of electronic and thermal Free Energies $=-3187.996082 E_{h}$ Number of imaginary frequencies: $1\left(v=-233.8 \mathrm{~cm}^{-1}\right)$

$\begin{array}{lccc}\text { K } & 0.20417 & 6.60411 & -0.54518 \\ \mathrm{Si} & -1.62306 & -0.57595 & 0.07156 \\ \mathrm{Si} & 1.79947 & -0.50568 & 0.02570 \\ \mathrm{Si} & 0.12162 & -2.02142 & 0.70824 \\ \mathrm{C} & -3.29230 & -1.98812 & -2.13445 \\ \mathrm{C} & -3.44810 & -0.17165 & 2.55137 \\ \mathrm{C} & 1.99264 & -1.81137 & -2.97170 \\ \mathrm{H} & 1.95982 & -0.74837 & -3.22554\end{array}$




\begin{tabular}{|c|c|c|c|}
\hline $\mathrm{H}$ & 1.98898 & -2.39630 & -3.89591 \\
\hline $\mathrm{H}$ & 1.08582 & -2.05818 & -2.40943 \\
\hline & -5.24781 & -1.62590 & 0.35669 \\
\hline C & -7.57458 & -1.14576 & -0.07482 \\
\hline $\mathrm{H}$ & -8.34038 & -0.47743 & -0.45679 \\
\hline C & -2.19434 & 0.48905 & 3.13013 \\
\hline $\mathrm{H}$ & -1.90780 & 1.36348 & 2.54134 \\
\hline $\mathrm{H}$ & -2.38612 & 0.80925 & 4.15838 \\
\hline $\mathrm{H}$ & -1.35255 & -0.21213 & 3.13781 \\
\hline C & -3.89614 & -0.89459 & -3.02545 \\
\hline $\mathrm{H}$ & -3.23800 & -0.02205 & -3.05485 \\
\hline $\mathrm{H}$ & -4.03434 & -1.26970 & -4.04479 \\
\hline $\mathrm{H}$ & -4.87109 & -0.58084 & -2.64065 \\
\hline C & 4.48813 & -1.80114 & -2.98042 \\
\hline $\mathrm{H}$ & 5.40791 & -2.10395 & -2.47659 \\
\hline $\mathrm{H}$ & 4.43971 & -2.32900 & -3.93802 \\
\hline $\mathrm{H}$ & 4.53505 & -0.72629 & -3.17928 \\
\hline C & 3.24271 & -2.13749 & -2.15099 \\
\hline C & -5.60154 & -2.86026 & 0.91008 \\
\hline $\mathrm{H}$ & -4.82774 & -3.52665 & 1.27818 \\
\hline C & 5.37663 & -1.74393 & 0.18675 \\
\hline C & -4.58813 & 0.85486 & 2.51181 \\
\hline $\mathrm{H}$ & -5.51980 & 0.40614 & 2.15924 \\
\hline $\mathrm{H}$ & -4.76306 & 1.25279 & 3.51649 \\
\hline $\mathrm{H}$ & -4.32515 & 1.68479 & 1.84910 \\
\hline C & -4.22355 & -3.20641 & -2.07991 \\
\hline $\mathrm{H}$ & -5.25127 & -2.93586 & -1.83266 \\
\hline $\mathrm{H}$ & -4.23286 & -3.68386 & -3.06434 \\
\hline $\mathrm{H}$ & -3.86834 & -3.93689 & -1.34693 \\
\hline C & -1.94006 & -2.43150 & -2.70252 \\
\hline $\mathrm{H}$ & -1.47859 & -3.19297 & -2.06575 \\
\hline $\mathrm{H}$ & -2.07776 & -2.84776 & -3.70476 \\
\hline $\mathrm{H}$ & -1.25406 & -1.58400 & -2.77995 \\
\hline C & -3.82887 & -1.37606 & 3.42233 \\
\hline $\mathrm{H}$ & -3.03950 & -2.13334 & 3.39081 \\
\hline $\mathrm{H}$ & -3.95812 & -1.05324 & 4.46024 \\
\hline $\mathrm{H}$ & -4.76558 & -1.83054 & 3.09384 \\
\hline C & -6.23471 & -0.76717 & -0.13650 \\
\hline $\mathrm{H}$ & -5.95186 & 0.19270 & -0.55747 \\
\hline C & 3.22958 & -3.62306 & -1.76615 \\
\hline $\mathrm{H}$ & 2.35689 & -3.84132 & -1.14186 \\
\hline $\mathrm{H}$ & 3.17961 & -4.24362 & -2.66666 \\
\hline $\mathrm{H}$ & 4.13268 & -3.89807 & -1.21506 \\
\hline C & 6.42578 & -0.99545 & -0.35688 \\
\hline $\mathrm{H}$ & 6.21556 & -0.04162 & -0.83075 \\
\hline C & 7.98825 & -2.71381 & 0.30903 \\
\hline $\mathrm{H}$ & 9.00536 & -3.09164 & 0.35634 \\
\hline C & 5.63566 & -2.97781 & 0.79185 \\
\hline $\mathrm{H}$ & 4.81646 & -3.55060 & 1.21570 \\
\hline C & -7.92858 & -2.37788 & 0.47534 \\
\hline $\mathrm{H}$ & -8.97311 & -2.67184 & 0.52043 \\
\hline
\end{tabular}




\begin{tabular}{|c|c|c|c|}
\hline C & 7.72992 & -1.48042 & -0.29038 \\
\hline $\mathrm{H}$ & 8.54302 & -0.89585 & -0.71009 \\
\hline B & 1.37055 & 1.96227 & -1.91196 \\
\hline $\mathrm{H}$ & 2.24145 & 1.69915 & -2.69670 \\
\hline C & -6.94189 & -3.23318 & 0.96789 \\
\hline $\mathrm{H}$ & -7.21502 & -4.19353 & 1.39471 \\
\hline C & 6.94107 & -3.46184 & 0.84771 \\
\hline $\mathrm{H}$ & 7.13877 & -4.42223 & 1.31418 \\
\hline B & -0.19534 & 0.97578 & -1.80193 \\
\hline $\mathrm{H}$ & -0.17055 & -0.00086 & -2.49191 \\
\hline $\mathrm{N}$ & 3.16699 & -1.29661 & $-0.9425 c$ \\
\hline$N$ & -3.02437 & -1.42183 & -0.8009 \\
\hline$N$ & -3.09937 & -0.59910 & 1.18612 \\
\hline C & -3.82311 & -1.23053 & 0.2545 \\
\hline C & 3.98733 & -1.23080 & 0.1167 \\
\hline B & 0.11660 & 1.64982 & 0.43273 \\
\hline $\mathrm{H}$ & 0.09183 & 1.34610 & 1.59702 \\
\hline B & -1.39166 & 2.61377 & -0.02240 \\
\hline $\mathrm{H}$ & -2.27042 & 2.85809 & 0.75655 \\
\hline B & 1.74998 & 2.69569 & -0.31864 \\
\hline $\mathrm{H}$ & 2.82294 & 3.13675 & 0.01280 \\
\hline C & 1.36497 & 1.16943 & -0.45084 \\
\hline C & -1.22676 & 1.06754 & -0.57400 \\
\hline B & -1.68940 & 2.14241 & -1.71042 \\
\hline $\mathrm{H}$ & -2.76751 & 2.12113 & -2.2345 \\
\hline B & -0.17131 & 2.57941 & -2.5685 \\
\hline B & -0.90000 & 3.67292 & -1.34532 \\
\hline B & 0.86712 & 3.62712 & -1.51534 \\
\hline B & 0.15149 & 3.48625 & 0.11439 \\
\hline $\mathrm{H}$ & -0.17881 & 2.78262 & -3.75151 \\
\hline $\mathrm{H}$ & -1.49425 & 4.68590 & -1.6211 \\
\hline $\mathrm{H}$ & 1.41928 & 4.60819 & $-1.950 €$ \\
\hline $\mathrm{H}$ & 0.15184 & 4.34970 & 0.95795 \\
\hline C & 3.77980 & -0.03429 & 2.36303 \\
\hline$N$ & 3.31352 & -0.62033 & 1.09511 \\
\hline C & 4.30222 & -1.12385 & 3.30746 \\
\hline $\mathrm{H}$ & 4.52129 & -0.68713 & 4.28700 \\
\hline $\mathrm{H}$ & 3.54975 & -1.90795 & 3.43674 \\
\hline $\mathrm{H}$ & 5.21966 & -1.57976 & 2.92961 \\
\hline C & 2.56103 & 0.64488 & 2.99314 \\
\hline $\mathrm{H}$ & 2.19487 & 1.45139 & 2.35237 \\
\hline $\mathrm{H}$ & 1.74655 & -0.07233 & 3.14291 \\
\hline $\mathrm{H}$ & 2.83215 & 1.06788 & 3.96478 \\
\hline C & 4.85973 & 1.02129 & 2.09129 \\
\hline $\mathrm{H}$ & 5.12306 & 1.53205 & 3.02310 \\
\hline $\mathrm{H}$ & 5.76780 & 0.56731 & 1.6869 \\
\hline $\mathrm{H}$ & 4.48644 & 1.76303 & 1.3785 \\
\hline
\end{tabular}

Calculated cartesian coordinates and energies of $E$

$E(U B 3 P W 91)=\quad-3188.783150 E_{h}$

Sum of electronic and zero-point Energies $=\quad-3187.913050 E_{h}$ 
$\begin{array}{ll}\text { Sum of electronic and thermal Energies }= & -3187.860449 E_{h} \\ \text { Sum of electronic and thermal Enthalpies }= & -3187.859505 E_{h} \\ \text { Sum of electronic and thermal Free Energies }= & -3187.998627 E_{h} \\ \text { Number of imaginary frequencies: } 0 & \end{array}$

$\begin{array}{llll}\mathrm{K} & 0.26935 & 6.64849 & -0.37619 \\ \mathrm{Si} & -1.62045 & -0.56844 & 0.02489 \\ \mathrm{Si} & 1.80569 & -0.47701 & 0.03394 \\ \mathrm{Si} & 0.12001 & -2.03205 & 0.59555 \\ \mathrm{C} & -3.32876 & -1.85383 & -2.22918 \\ \mathrm{C} & -3.36126 & -0.40502 & 2.58300 \\ \mathrm{C} & 1.95325 & -1.61050 & -3.03406 \\ \mathrm{H} & 1.87852 & -0.53235 & -3.19627 \\ \mathrm{H} & 1.95227 & -2.11651 & -4.00385 \\ \mathrm{H} & 1.06653 & -1.93487 & -2.47999 \\ \mathrm{C} & -5.25581 & -1.58442 & 0.30753 \\ \mathrm{C} & -7.56848 & -0.98403 & -0.04050 \\ \mathrm{H} & -8.31362 & -0.26225 & -0.36131 \\ \mathrm{C} & -2.15634 & 0.35286 & 3.14605 \\ \mathrm{H} & -2.02785 & 1.31255 & 2.64021 \\ \mathrm{H} & -2.30200 & 0.53572 & 4.21468 \\ \mathrm{H} & -1.23559 & -0.22734 & 3.01969 \\ \mathrm{C} & -3.78726 & -0.66677 & -3.08619 \\ \mathrm{H} & -3.02897 & 0.12005 & -3.09022 \\ \mathrm{H} & -3.96646 & -0.99154 & -4.11641 \\ \mathrm{H} & -4.71799 & -0.24824 & -2.69019 \\ \mathrm{C} & 4.44897 & -1.52789 & -3.08169 \\ \mathrm{H} & 5.38469 & -1.82579 & -2.60426 \\ \mathrm{H} & 4.41153 & -1.99420 & -4.07130 \\ \mathrm{H} & 4.45106 & -0.44121 & -3.21004 \\ \mathrm{C} & 3.22764 & -1.96479 & -2.26355 \\ \mathrm{C} & -5.64824 & -2.83839 & 0.78310 \\ \mathrm{H} & -4.89656 & -3.56284 & 1.08024 \\ \mathrm{C} & 5.36214 & -1.79034 & 0.10531 \\ \mathrm{C} & -4.62441 & 0.44335 & 2.77714 \\ \mathrm{H} & -5.52451 & -0.08774 & 2.46083 \\ \mathrm{H} & -4.73564 & 0.69534 & 3.83649 \\ \mathrm{H} & -4.54794 & 1.37312 & 2.20513 \\ \mathrm{C} & -4.38795 & -2.96309 & -2.25537 \\ \mathrm{H} & -5.38671 & -2.59500 & -2.01564 \\ \mathrm{H} & -4.42152 & -3.38432 & -3.26469 \\ \mathrm{H} & -4.13373 & -3.76652 & -1.55768 \\ \mathrm{C} & -2.01717 & -2.42721 & -2.77783 \\ \mathrm{H} & -1.67858 & -3.27286 & -2.17107 \\ \mathrm{H} & -2.16365 & -2.76820 & -3.80699 \\ \mathrm{H} & -3.23195 & -1.66786 & -2.78201 \\ \mathrm{H} & -0.65575 & -1.75260 & 3.30617 \\ \mathrm{H} & -0.10626\end{array}$




\begin{tabular}{|c|c|c|c|}
\hline $\mathrm{H}$ & -5.90112 & 0.31767 & -0.46961 \\
\hline$c$ & 3.25746 & -3.47579 & -1.99846 \\
\hline $\mathrm{H}$ & 2.40807 & -3.76134 & -1.36901 \\
\hline $\mathrm{H}$ & 3.19174 & -4.02312 & -2.94424 \\
\hline $\mathrm{H}$ & 4.18173 & -3.77543 & -1.49808 \\
\hline C & 6.41563 & -0.99503 & -0.35608 \\
\hline $\mathrm{H}$ & 6.20805 & -0.00836 & -0.75881 \\
\hline C & 7.97733 & -2.75114 & 0.20460 \\
\hline $\mathrm{H}$ & 8.99608 & -3.12560 & 0.24363 \\
\hline C & 5.61789 & -3.06647 & 0.61769 \\
\hline $\mathrm{H}$ & 4.79510 & -3.67200 & 0.98508 \\
\hline C & -7.96214 & -2.23479 & 0.43687 \\
\hline $\mathrm{H}$ & -9.01684 & -2.48896 & 0.48689 \\
\hline C & 7.72225 & -1.47647 & -0.30164 \\
\hline $\mathrm{H}$ & 8.53940 & -0.85614 & -0.65735 \\
\hline B & 1.25776 & 1.92460 & -1.80000 \\
\hline $\mathrm{H}$ & 2.11076 & 1.71686 & -2.62592 \\
\hline C & -7.00191 & -3.15981 & 0.84816 \\
\hline $\mathrm{H}$ & -7.30570 & -4.13538 & 1.21565 \\
\hline C & 6.92472 & -3.54576 & 0.66119 \\
\hline $\mathrm{H}$ & 7.12090 & -4.53820 & 1.05580 \\
\hline B & -0.45127 & 1.08044 & -1.95016 \\
\hline $\mathrm{H}$ & -0.50118 & 0.15246 & -2.70364 \\
\hline$N$ & 3.16066 & -1.22301 & -0.99042 \\
\hline$N$ & -3.03920 & -1.37293 & -0.86601 \\
\hline$N$ & -3.08794 & -0.62110 & 1.15159 \\
\hline C & -3.82356 & -1.21784 & 0.20511 \\
\hline C & 3.97556 & -1.26846 & 0.07328 \\
\hline B & 0.14985 & 1.68582 & 0.52747 \\
\hline $\mathrm{H}$ & 0.07113 & 1.36540 & 1.68581 \\
\hline B & -1.37012 & 2.59295 & 0.09261 \\
\hline $\mathrm{H}$ & -2.21742 & 2.78547 & 0.91949 \\
\hline B & 1.76547 & 2.73727 & -0.20927 \\
\hline $\mathrm{H}$ & 2.81088 & 3.27042 & 0.09052 \\
\hline C & 1.42664 & 1.23781 & -0.30408 \\
\hline C & -1.22728 & 1.10003 & -0.57781 \\
\hline B & -1.76945 & 2.25311 & -1.61699 \\
\hline $\mathrm{H}$ & -2.87798 & 2.31061 & -2.06780 \\
\hline B & -0.24171 & 2.70339 & -2.47742 \\
\hline B & -0.92020 & 3.75244 & -1.18515 \\
\hline B & 0.82681 & 3.66531 & -1.39456 \\
\hline B & 0.13900 & 3.52032 & 0.22558 \\
\hline $\mathrm{H}$ & -0.20855 & 2.99488 & -3.64194 \\
\hline $\mathrm{H}$ & -1.51200 & 4.78148 & -1.39544 \\
\hline $\mathrm{H}$ & 1.39214 & 4.63797 & -1.83184 \\
\hline $\mathrm{H}$ & 0.15136 & 4.35711 & 1.09564 \\
\hline C & 3.78095 & -0.19047 & 2.37887 \\
\hline$N$ & 3.30300 & -0.75361 & 1.10581 \\
\hline C & 4.62587 & -1.19564 & 3.17062 \\
\hline $\mathrm{H}$ & 4.81052 & -0.79777 & 4.17342 \\
\hline $\mathrm{H}$ & 4.09903 & -2.14969 & 3.26845 \\
\hline
\end{tabular}




$\begin{array}{llll}\mathrm{H} & 5.59372 & -1.37875 & 2.70028 \\ \mathrm{C} & 2.52405 & 0.15584 & 3.18314 \\ \mathrm{H} & 1.90693 & 0.88386 & 2.64808 \\ \mathrm{H} & 1.91952 & -0.73940 & 3.36197 \\ \mathrm{H} & 2.80466 & 0.58826 & 4.14801 \\ \mathrm{C} & 4.58359 & 1.08633 & 2.09347 \\ \mathrm{H} & 4.87527 & 1.56944 & 3.03187 \\ \mathrm{H} & 5.49442 & 0.85269 & 1.53376 \\ \mathrm{H} & 3.97961 & 1.78390 & 1.50583\end{array}$

Calculated cartesian coordinates and energies of TS(E-F)

$\mathrm{E}(\mathrm{UB} 3 \mathrm{PW} 91)=$ $-3188.782994 E_{h}$ Sum of electronic and zero-point Energies $=\quad-3187.912877 \mathrm{E}_{\mathrm{h}}$ Sum of electronic and thermal Energies $=\quad-3187.861021 \mathrm{E}_{\mathrm{h}}$ Sum of electronic and thermal Enthalpies $=\quad-3187.860077 E_{h}$ Sum of electronic and thermal Free Energies $=\quad-3187.996920 \mathrm{Eh}_{\mathrm{h}}$ Number of imaginary frequencies: $1\left(v=-138.3 \mathrm{~cm}^{-1}\right)$

$\begin{array}{llll}\text { K } & 0.30596 & 6.66778 & -0.35952 \\ \mathrm{Si} & -1.63423 & -0.55056 & 0.00261 \\ \mathrm{Si} & 1.80047 & -0.47813 & 0.04068 \\ \mathrm{Si} & 0.10053 & -2.01666 & 0.59090 \\ \mathrm{C} & -3.35507 & -1.82404 & -2.24281 \\ \mathrm{C} & -3.34801 & -0.41147 & 2.57733 \\ \mathrm{C} & 1.93396 & -1.58576 & -3.04161 \\ \mathrm{H} & 1.86874 & -0.50567 & -3.19396 \\ \mathrm{H} & 1.92865 & -2.08268 & -4.01608 \\ \mathrm{H} & 1.04424 & -1.90740 & -2.49090 \\ \mathrm{C} & -5.26126 & -1.58820 & 0.31479 \\ \mathrm{C} & -7.58032 & -0.99071 & 0.00628 \\ \mathrm{H} & -8.33208 & -0.26582 & -0.29119 \\ \mathrm{C} & -2.13843 & 0.34370 & 3.13442 \\ \mathrm{H} & -2.02278 & 1.31290 & 2.64367 \\ \mathrm{H} & -2.26810 & 0.50729 & 4.20824 \\ \mathrm{H} & -1.21694 & -0.22972 & 2.98381 \\ \mathrm{C} & -3.82013 & -0.61651 & -3.06757 \\ \mathrm{H} & -3.06237 & 0.17087 & -3.05713 \\ \mathrm{H} & -4.00670 & -0.91568 & -4.10425 \\ \mathrm{H} & -4.74836 & -0.20869 & -2.65469 \\ \mathrm{C} & 4.42969 & -1.52454 & -3.09048 \\ \mathrm{H} & 5.36346 & -1.83310 & -2.61605 \\ \mathrm{H} & 4.38785 & -1.98339 & -4.08338 \\ \mathrm{H} & 4.44008 & -0.43700 & -3.21109 \\ \mathrm{C} & 3.20529 & -1.95764 & -2.27482 \\ \mathrm{C} & -5.64302 & -2.85340 & 0.76882 \\ \mathrm{H} & -4.88475 & -3.58019 & 1.04263 \\ \mathrm{C} & 5.34804 & -1.81719 & 0.08615 \\ \mathrm{C} & -4.61219 & 0.42793 & 2.80091 \\ \mathrm{H} & -5.51389 & -0.10283 & 2.48852 \\ \mathrm{H} & -4.71040 & 0.66380 & 3.86526 \\ \mathrm{H} & -4.54775 & 1.36634 & 2.24160\end{array}$




\begin{tabular}{|c|c|c|c|}
\hline C & -4.41573 & -2.93131 & -2.28310 \\
\hline 1 & -5.41145 & -2.56696 & -2.02551 \\
\hline $\mathrm{H}$ & -4.45983 & -3.33086 & -3.30078 \\
\hline $\mathrm{H}$ & -4.15584 & -3.74955 & -1.60499 \\
\hline C & -2.04844 & -2.38520 & -2.81498 \\
\hline $\mathrm{H}$ & -1.70605 & -3.24437 & -2.22977 \\
\hline $\mathrm{H}$ & -2.20313 & -2.70311 & -3.85028 \\
\hline $\mathrm{H}$ & -1.26279 & -1.62606 & -2.80867 \\
\hline C & -3.45896 & -1.77028 & 3.28188 \\
\hline $\mathrm{H}$ & -2.57935 & -2.38188 & 3.05595 \\
\hline $\mathrm{H}$ & -3.51582 & -1.62661 & 4.36561 \\
\hline $\mathrm{H}$ & -4.35451 & -2.31056 & 2.96721 \\
\hline C & -6.23028 & -0.65505 & -0.06770 \\
\hline $\mathrm{H}$ & -5.92339 & 0.32714 & -0.41420 \\
\hline C & 3.22207 & -3.47105 & -2.02256 \\
\hline $\mathrm{H}$ & 2.37168 & -3.75462 & -1.39358 \\
\hline $\mathrm{H}$ & 3.14937 & -4.00968 & -2.97281 \\
\hline $\mathrm{H}$ & 4.14475 & -3.78333 & -1.52713 \\
\hline C & 6.40574 & -1.01981 & -0.36173 \\
\hline $\mathrm{H}$ & 6.20324 & -0.02599 & -0.74916 \\
\hline C & 7.95810 & -2.79240 & 0.17229 \\
\hline $\mathrm{H}$ & 8.97495 & -3.17245 & 0.20641 \\
\hline C & 5.59676 & -3.10263 & 0.57809 \\
\hline $\mathrm{H}$ & 4.77077 & -3.70953 & 0.93586 \\
\hline C & -7.96329 & -2.25266 & 0.46238 \\
\hline $\mathrm{H}$ & -9.01629 & -2.51234 & 0.51934 \\
\hline C & 7.70990 & -1.50865 & -0.31401 \\
\hline $\mathrm{H}$ & 8.53047 & -0.88694 & -0.65927 \\
\hline B & 1.27118 & 1.93757 & -1.77180 \\
\hline $\mathrm{H}$ & 2.12557 & 1.73084 & -2.59685 \\
\hline C & -6.99456 & -3.18163 & 0.84387 \\
\hline $\mathrm{H}$ & -7.29009 & -4.16556 & 1.19548 \\
\hline C & 6.90111 & -3.58901 & 0.61514 \\
\hline $\mathrm{H}$ & 7.09207 & -4.58847 & 0.99427 \\
\hline B & -0.46256 & 1.10378 & -1.96301 \\
\hline $\mathrm{H}$ & -0.49240 & 0.18769 & -2.73260 \\
\hline$N$ & 3.14613 & -1.22567 & -0.99528 \\
\hline$N$ & -3.05201 & -1.37494 & -0.87212 \\
\hline $\mathrm{N}$ & -3.09435 & -0.60507 & 1.13889 \\
\hline C & -3.83163 & -1.21445 & 0.20150 \\
\hline C & 3.96499 & -1.28553 & 0.06440 \\
\hline B & 0.23356 & 1.75330 & 0.58880 \\
\hline $\mathrm{H}$ & 0.13510 & 1.41037 & 1.73667 \\
\hline B & -1.34355 & 2.57271 & 0.09870 \\
\hline $\mathrm{H}$ & -2.18789 & 2.76879 & 0.92942 \\
\hline B & 1.76510 & 2.74636 & -0.16867 \\
\hline $\mathrm{H}$ & 2.80400 & 3.27009 & 0.16456 \\
\hline C & 1.42993 & 1.23851 & -0.29466 \\
\hline C & -1.26383 & 1.11056 & -0.62333 \\
\hline B & -1.77091 & 2.29049 & -1.62549 \\
\hline $\mathrm{H}$ & -2.87442 & 2.40982 & -2.08067 \\
\hline
\end{tabular}




$\begin{array}{llll}\text { B } & -0.22099 & 2.73277 & -2.46373 \\ \text { B } & -0.89753 & 3.76847 & -1.16239 \\ \text { B } & 0.84536 & 3.68032 & -1.37443 \\ \text { B } & 0.15635 & 3.53485 & 0.24893 \\ \text { H } & -0.17306 & 3.03675 & -3.62499 \\ \text { H } & -1.48333 & 4.80471 & -1.35404 \\ \text { H } & 1.41760 & 4.64922 & -1.81031 \\ \text { H } & 0.14694 & 4.37798 & 1.11286 \\ \text { C } & 3.79139 & -0.22983 & 2.38145 \\ \text { N } & 3.30162 & -0.77374 & 1.10433 \\ \text { C } & 4.66009 & -1.23776 & 3.14386 \\ \text { H } & 4.85025 & -0.85503 & 4.15151 \\ \text { H } & 4.14829 & -2.20099 & 3.23089 \\ \text { H } & 5.62532 & -1.39809 & 2.66016 \\ \text { C } & 2.54330 & 0.08573 & 3.21138 \\ \text { H } & 1.90684 & 0.81284 & 2.69831 \\ \text { H } & 1.95549 & -0.82122 & 3.38658 \\ \text { H } & 2.83380 & 0.50787 & 4.17787 \\ \text { C } & 4.57605 & 1.06054 & 2.10755 \\ \text { H } & 4.87802 & 1.52931 & 3.04996 \\ \text { H } & 5.47984 & 0.84589 & 1.52903 \\ \text { H } & 3.95697 & 1.76292 & 1.54201\end{array}$

\section{Calculated cartesian coordinates and energies of $F$}

$\begin{array}{ll}\text { E(UB3PW91) = } & -3188.784913 E_{h} \\ \text { Sum of electronic and zero-point Energies }= & -3187.914746 E_{h} \\ \text { Sum of electronic and thermal Energies= } & -3187.862133 E_{h} \\ \text { Sum of electronic and thermal Enthalpies= } & -3187.861189 E_{h} \\ \text { Sum of electronic and thermal Free Energies }= & -3188.001191 E_{h} \\ \text { Number of imaginary frequencies: } 0 & \end{array}$

$\begin{array}{lrrr}\text { K } & 0.34540 & 6.97958 & 0.03971 \\ \mathrm{Si} & -1.74097 & -0.25165 & -0.08915 \\ \mathrm{Si} & 1.71615 & -0.23839 & 0.04832 \\ \mathrm{Si} & -0.02120 & -1.79316 & 0.33934 \\ \mathrm{C} & -3.55550 & -1.35629 & -2.33124 \\ \mathrm{C} & -3.32735 & -0.28367 & 2.56431 \\ \mathrm{C} & 1.99168 & -1.07380 & -3.10032 \\ \mathrm{H} & 1.82968 & 0.00614 & -3.12433 \\ \mathrm{H} & 2.05634 & -1.44130 & -4.12857 \\ \mathrm{H} & 1.12244 & -1.53989 & -2.62266 \\ \mathrm{C} & -5.33781 & -1.36431 & 0.32902 \\ \mathrm{C} & -7.68077 & -0.79387 & 0.20287 \\ \mathrm{H} & -8.46060 & -0.05990 & 0.02320 \\ \mathrm{C} & -2.07180 & 0.40144 & 3.11184 \\ \mathrm{H} & -1.94781 & 1.39558 & 2.67679 \\ \mathrm{H} & -2.14893 & 0.49967 & 4.19874 \\ \mathrm{H} & -1.17409 & -0.18435 & 2.88493 \\ \mathrm{C} & -4.08790 & -0.07332 & -2.98356 \\ \mathrm{H} & -3.35149 & 0.73079 & -2.90509 \\ \mathrm{H} & -4.31267 & -0.24905 & -4.04065\end{array}$




\begin{tabular}{|c|c|c|c|}
\hline $\mathrm{H}$ & -5.00721 & 0.25055 & -2.48509 \\
\hline C & 4.46736 & -0.73486 & -3.03238 \\
\hline$\Pi$ & 5.41319 & -0.99437 & -2.55109 \\
\hline $\mathrm{H}$ & 4.52063 & -1.05182 & -4.07875 \\
\hline $\mathrm{H}$ & 4.34540 & 0.35214 & -3.00367 \\
\hline$c$ & 3.27830 & -1.41742 & -2.34446 \\
\hline C & -5.67174 & -2.68037 & 0.66044 \\
\hline $\mathrm{H}$ & -4.88544 & -3.41174 & 0.81863 \\
\hline C & 5.28242 & -1.51393 & 0.14263 \\
\hline C & -4.55569 & 0.57113 & 2.90179 \\
\hline $\mathrm{H}$ & -5.48358 & 0.08812 & 2.58767 \\
\hline $\mathrm{H}$ & -4.60648 & 0.73098 & 3.98346 \\
\hline $\mathrm{H}$ & -4.48475 & 1.54551 & 2.40871 \\
\hline C & -4.59433 & -2.47890 & -2.44276 \\
\hline $\mathrm{H}$ & -5.58046 & -2.17102 & -2.09092 \\
\hline $\mathrm{H}$ & -4.68902 & -2.76314 & -3.49519 \\
\hline $\mathrm{H}$ & -4.28032 & -3.36016 & -1.87564 \\
\hline C & -2.26605 & -1.81097 & -3.02466 \\
\hline $\mathrm{H}$ & -1.87241 & -2.71486 & -2.54918 \\
\hline $\mathrm{H}$ & -2.46429 & -2.02411 & -4.07928 \\
\hline $\mathrm{H}$ & -1.49815 & -1.03370 & -2.97458 \\
\hline C & -3.44742 & -1.68339 & 3.18174 \\
\hline $\mathrm{H}$ & -2.59129 & -2.29909 & 2.88716 \\
\hline $\mathrm{H}$ & -3.46365 & -1.60878 & 4.27375 \\
\hline $\mathrm{H}$ & -4.36608 & -2.18241 & 2.86497 \\
\hline C & -6.34274 & -0.41874 & 0.10136 \\
\hline $\mathrm{H}$ & -6.07274 & 0.60257 & -0.14940 \\
\hline C & 3.45850 & -2.94126 & -2.32327 \\
\hline $\mathrm{H}$ & 2.63703 & -3.41164 & -1.77316 \\
\hline $\mathrm{H}$ & 3.45682 & -3.32820 & -3.34720 \\
\hline $\mathrm{H}$ & 4.40385 & -3.22912 & -1.85799 \\
\hline C & 6.32277 & -0.60676 & -0.07958 \\
\hline $\mathrm{H}$ & 6.09290 & 0.42916 & -0.30960 \\
\hline C & 7.92865 & -2.37492 & 0.28397 \\
\hline $\mathrm{H}$ & 8.95999 & -2.71061 & 0.34048 \\
\hline C & 5.56496 & -2.85170 & 0.43440 \\
\hline $\mathrm{H}$ & 4.75024 & -3.54544 & 0.61725 \\
\hline C & -8.01551 & -2.10712 & 0.53488 \\
\hline $\mathrm{H}$ & -9.05909 & -2.39751 & 0.61337 \\
\hline C & 7.64535 & -1.04002 & -0.00612 \\
\hline $\mathrm{H}$ & 8.45305 & -0.33453 & -0.17650 \\
\hline B & 1.28298 & 2.31068 & -1.57947 \\
\hline $\mathrm{H}$ & 2.12791 & 2.11252 & -2.41481 \\
\hline C & -7.01101 & -3.04783 & 0.76606 \\
\hline $\mathrm{H}$ & -7.26938 & -4.07070 & 1.02313 \\
\hline C & 6.88832 & -3.27973 & 0.50099 \\
\hline $\mathrm{H}$ & 7.10688 & -4.31903 & 0.72722 \\
\hline B & -0.47146 & 1.50105 & -1.87657 \\
\hline $\mathrm{H}$ & -0.43406 & 0.65430 & -2.72509 \\
\hline $\mathrm{N}$ & 3.11105 & -0.89095 & -0.97820 \\
\hline $\mathrm{N}$ & -3.18701 & -1.06048 & -0.93720 \\
\hline
\end{tabular}




$\begin{array}{llll}\text { N } & -3.15009 & -0.37868 & 1.10452 \\ \text { C } & -3.92159 & -0.95587 & 0.17369 \\ \text { C } & 3.88195 & -1.03104 & 0.10763 \\ \text { B } & 0.44246 & 2.11206 & 0.89030 \\ \text { H } & 0.38208 & 1.71585 & 2.02076 \\ \text { B } & -1.29326 & 2.75347 & 0.27598 \\ \text { H } & -2.13487 & 2.94285 & 1.11637 \\ \text { B } & 1.78300 & 3.03635 & 0.03998 \\ \text { H } & 2.84612 & 3.49174 & 0.38162 \\ \text { C } & 1.38912 & 1.51391 & -0.16924 \\ \text { C } & -1.39614 & 1.42287 & -0.64508 \\ \text { B } & -1.78605 & 2.71174 & -1.49470 \\ \text { H } & -2.85309 & 2.97839 & -1.98525 \\ \text { B } & -0.19439 & 3.17320 & -2.25613 \\ \text { B } & -0.86151 & 4.10036 & -0.88392 \\ \text { B } & 0.87152 & 4.03282 & -1.12200 \\ \text { B } & 0.19601 & 3.79155 & 0.51414 \\ \text { H } & -0.12387 & 3.55620 & -3.39346 \\ \text { H } & -1.43510 & 5.15735 & -0.97709 \\ \text { H } & 1.45299 & 5.01656 & -1.50777 \\ \text { H } & 0.14068 & 4.61305 & 1.39818 \\ \text { C } & 3.54164 & -0.29086 & 2.53728 \\ \text { N } & 3.15830 & -0.63328 & 1.15839 \\ \text { C } & 4.56005 & -1.27143 & 3.13111 \\ \text { H } & 4.66789 & -1.05921 & 4.19925 \\ \text { H } & 4.21892 & -2.30470 & 3.01855 \\ \text { H } & 5.54540 & -1.17790 & 2.67122 \\ \text { C } & 2.25418 & -0.35690 & 3.36657 \\ \text { H } & 1.49854 & 0.32882 & 2.97247 \\ \text { H } & 1.83886 & -1.36933 & 3.35469 \\ \text { H } & 2.46298 & -0.07319 & 4.40239 \\ \text { C } & 4.10752 & 1.13600 & 2.54989 \\ \text { H } & 4.34510 & 1.44288 & 3.57383 \\ \text { H } & 5.02480 & 1.18592 & 1.95413 \\ \text { H } & 3.38297 & 1.83657 & 2.12613\end{array}$

\section{Calculated cartesian coordinates and energies of 4}

\section{$\mathrm{E}(\mathrm{RB} 3 \mathrm{PW} 91)=$}

Sum of electronic and zero-point Energies=

Sum of electronic and thermal Energies=

Sum of electronic and thermal Enthalpies=

Sum of electronic and thermal Free Energies=

Number of imaginary frequencies: 0

$\begin{array}{lrrl} & & & \\ \text { K } & -8.48244 & -3.41641 & -0.92865 \\ \mathrm{Si} & 2.52107 & 1.93027 & 1.05979 \\ \mathrm{Si} & 3.24379 & -1.01641 & -0.67330 \\ \mathrm{~K} & 9.23599 & 3.44858 & -1.09231 \\ \mathrm{Si} & 1.60895 & -0.18767 & 0.75657 \\ \mathrm{C} & 1.81574 & 4.39294 & -0.73105 \\ \mathrm{C} & 2.21757 & 2.75930 & 4.00261\end{array}$

$-6377.652684 E_{h}$ $-6375.907049 E_{h}$ $-6375.802254 E_{h}$ $-6375.801309 E_{h}$ $-6376.041893 E_{h}$ 


\begin{tabular}{|c|c|c|c|}
\hline C & 2.15922 & 0.36429 & -3.45993 \\
\hline $\mathrm{H}$ & 3.00492 & 0.94258 & -3.07736 \\
\hline$H$ & 1.81609 & 0.82407 & -4.39135 \\
\hline $\mathrm{H}$ & 1.34702 & 0.40823 & -2.72649 \\
\hline$c$ & 1.69284 & 5.39120 & 2.29642 \\
\hline C & 2.71838 & 7.54424 & 2.67318 \\
\hline $\mathrm{H}$ & 3.59225 & 8.18817 & 2.64063 \\
\hline C & 2.57545 & 1.28854 & 4.23921 \\
\hline $\mathrm{H}$ & 3.49135 & 1.01761 & 3.70354 \\
\hline $\mathrm{H}$ & 2.73713 & 1.11731 & 5.30794 \\
\hline $\mathrm{H}$ & 1.77251 & 0.63145 & 3.89795 \\
\hline C & 3.21783 & 4.81235 & -1.19480 \\
\hline $\mathrm{H}$ & 3.86364 & 3.94754 & -1.34260 \\
\hline $\mathrm{H}$ & 3.13878 & 5.36085 & -2.13996 \\
\hline $\mathrm{H}$ & 3.68610 & 5.46537 & -0.45382 \\
\hline C & 3.72806 & -1.12853 & -4.71321 \\
\hline $\mathrm{H}$ & 4.01930 & -2.15515 & -4.94843 \\
\hline $\mathrm{H}$ & 3.43033 & -0.63753 & -5.64558 \\
\hline $\mathrm{H}$ & 4.59607 & -0.60406 & -4.30259 \\
\hline C & 2.56706 & -1.08761 & -3.71208 \\
\hline C & 0.47546 & 5.88271 & 2.76950 \\
\hline $\mathrm{H}$ & -0.39126 & 5.23287 & 2.79577 \\
\hline C & 3.66151 & -3.97776 & -2.98821 \\
\hline C & 3.38011 & 3.64024 & 4.47992 \\
\hline $\mathrm{H}$ & 3.13596 & 4.70287 & 4.41670 \\
\hline $\mathrm{H}$ & 3.61185 & 3.40913 & 5.52475 \\
\hline $\mathrm{H}$ & 4.26947 & 3.44343 & 3.87417 \\
\hline C & 0.95134 & 5.65271 & -0.62380 \\
\hline $\mathrm{H}$ & 1.40665 & 6.42578 & -0.00388 \\
\hline $\mathrm{H}$ & 0.82166 & 6.06760 & -1.62817 \\
\hline $\mathrm{H}$ & -0.03517 & 5.42352 & -0.22218 \\
\hline C & 1.18099 & 3.43798 & -1.74675 \\
\hline $\mathrm{H}$ & 0.16924 & 3.16148 & -1.43871 \\
\hline $\mathrm{H}$ & 1.13815 & 3.90512 & -2.73561 \\
\hline $\mathrm{H}$ & 1.76463 & 2.51881 & -1.82437 \\
\hline C & 0.92966 & 3.08982 & 4.76647 \\
\hline $\mathrm{H}$ & 0.10013 & 2.48505 & 4.39028 \\
\hline $\mathrm{H}$ & 1.06435 & 2.87165 & 5.83086 \\
\hline $\mathrm{H}$ & 0.66596 & 4.14556 & 4.67099 \\
\hline C & 2.81877 & 6.22183 & 2.24958 \\
\hline $\mathrm{H}$ & 3.76171 & 5.82005 & 1.89038 \\
\hline C & 1.35025 & -1.85596 & -4.24761 \\
\hline $\mathrm{H}$ & 0.58601 & -1.93269 & -3.46722 \\
\hline $\mathrm{H}$ & 0.92314 & -1.32249 & -5.10273 \\
\hline $\mathrm{H}$ & 1.62166 & -2.85851 & -4.58347 \\
\hline C & 5.00170 & -4.13802 & -3.35802 \\
\hline $\mathrm{H}$ & 5.74787 & -3.44578 & -2.97941 \\
\hline C & 4.39609 & -6.06149 & -4.68848 \\
\hline $\mathrm{H}$ & 4.68172 & -6.87478 & -5.34925 \\
\hline C & 2.69104 & -4.85799 & -3.47026 \\
\hline $\mathrm{H}$ & 1.65741 & -4.73731 & -3.16724 \\
\hline
\end{tabular}




\begin{tabular}{|c|c|c|c|}
\hline 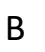 & 7.38576 & 0.89872 & -0.46268 \\
\hline $\mathrm{H}$ & 8.50767 & 0.78462 & -0.89272 \\
\hline 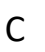 & 1.49772 & 8.04065 & 3.13577 \\
\hline $\mathrm{H}$ & 1.42056 & 9.07435 & 3.46006 \\
\hline C & 5.36542 & -5.17948 & -4.20848 \\
\hline $\mathrm{H}$ & 6.40578 & -5.30205 & -4.49469 \\
\hline B & 5.91645 & 0.61998 & -1.43277 \\
\hline $\mathrm{H}$ & 5.90102 & 0.42803 & -2.61684 \\
\hline B & 7.01091 & 2.11942 & 0.75482 \\
\hline $\mathrm{H}$ & 7.92674 & 2.77894 & 1.18592 \\
\hline C & 0.37871 & 7.20949 & 3.18429 \\
\hline $\mathrm{H}$ & -0.57183 & 7.59274 & 3.54344 \\
\hline C & 3.06054 & -5.89865 & -4.31928 \\
\hline $\mathrm{H}$ & 2.30408 & -6.58468 & -4.68862 \\
\hline B & 4.58738 & 1.54418 & -0.64638 \\
\hline $\mathrm{H}$ & 3.81324 & 1.87556 & -1.50939 \\
\hline$N$ & 2.98144 & -1.62843 & -2.40729 \\
\hline$N$ & 1.89471 & 3.62765 & 0.52584 \\
\hline$N$ & 1.99950 & 2.90308 & 2.55169 \\
\hline C & 1.82177 & 4.00090 & 1.80392 \\
\hline C & 3.33733 & -2.86936 & -2.05751 \\
\hline B & 5.30124 & -0.23949 & 1.06454 \\
\hline $\mathrm{H}$ & 4.84169 & -1.02863 & 1.84211 \\
\hline B & 5.58684 & 1.49976 & 1.74524 \\
\hline $\mathrm{H}$ & 5.58652 & 1.68933 & 2.93519 \\
\hline B & 6.90442 & 0.37378 & 1.18755 \\
\hline $\mathrm{H}$ & 7.67587 & -0.00411 & 2.02752 \\
\hline B & 6.46554 & -0.60524 & -0.26166 \\
\hline $\mathrm{H}$ & 6.96588 & -1.64340 & -0.58341 \\
\hline C & 4.87603 & -0.21794 & -0.46410 \\
\hline C & 4.30439 & 2.01064 & 0.84452 \\
\hline B & 5.47653 & 2.98293 & 0.64159 \\
\hline $\mathrm{H}$ & 5.56166 & 4.15720 & 0.93360 \\
\hline B & 6.31208 & 2.25799 & -0.86381 \\
\hline $\mathrm{H}$ & 6.62932 & 3.03450 & -1.73382 \\
\hline Si & -2.19968 & 0.75697 & -0.67622 \\
\hline Si & -1.48950 & -2.14772 & 1.11444 \\
\hline Si & -0.41312 & -0.68811 & -0.34399 \\
\hline C & -2.73070 & 3.30152 & 1.06979 \\
\hline C & -2.29717 & 1.59091 & -3.63424 \\
\hline C & -1.18765 & -0.35095 & 3.86876 \\
\hline $\mathrm{H}$ & -2.20009 & -0.23509 & 3.47207 \\
\hline $\mathrm{H}$ & -1.10880 & 0.23086 & 4.79174 \\
\hline $\mathrm{H}$ & -0.47755 & 0.05231 & 3.13828 \\
\hline C & -3.07358 & 4.18509 & -1.97087 \\
\hline C & -4.99009 & 5.59846 & -2.36969 \\
\hline $\mathrm{H}$ & -6.06428 & 5.75555 & -2.34283 \\
\hline C & -1.89868 & 0.12824 & -3.85728 \\
\hline $\mathrm{H}$ & -2.58078 & -0.55040 & -3.33567 \\
\hline $\mathrm{H}$ & -1.93402 & -0.10244 & -4.92660 \\
\hline $\mathrm{H}$ & -0.88687 & -0.05994 & -3.49160 \\
\hline
\end{tabular}




\begin{tabular}{|c|c|c|c|}
\hline C & -4.17039 & 3.03774 & 1.53151 \\
\hline $\mathrm{H}$ & -4.35076 & 1.97508 & 1.69040 \\
\hline$H$ & -4.35332 & 3.56996 & 2.47153 \\
\hline $\mathrm{H}$ & -4.88461 & 3.39863 & 0.78709 \\
\hline C & -1.90317 & -2.37665 & 5.15378 \\
\hline $\mathrm{H}$ & -1.68735 & -3.41714 & 5.40883 \\
\hline $\mathrm{H}$ & -1.87533 & -1.78771 & 6.07645 \\
\hline H & -2.91144 & -2.32076 & 4.73318 \\
\hline C & -0.88107 & -1.82142 & 4.15418 \\
\hline$C$ & -2.22835 & 5.18761 & -2.44858 \\
\hline $\mathrm{H}$ & -1.15743 & 5.02219 & -2.46718 \\
\hline C & -0.47438 & -4.90182 & 3.50314 \\
\hline C & -3.74047 & 1.79794 & -4.11353 \\
\hline $\mathrm{H}$ & -4.03372 & 2.84896 & -4.06764 \\
\hline $\mathrm{H}$ & -3.83557 & 1.46599 & -5.15276 \\
\hline $\mathrm{H}$ & -4.42607 & 1.21115 & -3.49504 \\
\hline C & -2.54305 & 4.81559 & 0.93346 \\
\hline $\mathrm{H}$ & -3.30081 & 5.28065 & 0.30195 \\
\hline $\mathrm{H}$ & -2.62401 & 5.26310 & 1.92884 \\
\hline $\mathrm{H}$ & -1.55975 & 5.06056 & 0.53301 \\
\hline C & -1.73479 & 2.76945 & 2.10495 \\
\hline $\mathrm{H}$ & -0.70574 & 2.96511 & 1.79123 \\
\hline $\mathrm{H}$ & -1.90815 & 3.24060 & 3.07752 \\
\hline $\mathrm{H}$ & -1.84620 & 1.69059 & 2.22377 \\
\hline C & -1.32798 & 2.49221 & -4.40881 \\
\hline $\mathrm{H}$ & -0.30888 & 2.36205 & -4.03450 \\
\hline $\mathrm{H}$ & -1.34507 & 2.22718 & -5.47097 \\
\hline $\mathrm{H}$ & -1.60267 & 3.54573 & -4.32144 \\
\hline C & -4.45824 & 4.38810 & -1.93325 \\
\hline $\mathrm{H}$ & -5.10327 & 3.59368 & -1.56915 \\
\hline C & 0.54421 & -1.92641 & 4.71397 \\
\hline $\mathrm{H}$ & 1.26900 & -1.65068 & 3.94164 \\
\hline $\mathrm{H}$ & 0.65901 & -1.24171 & 5.56024 \\
\hline $\mathrm{H}$ & 0.76521 & -2.93418 & 5.06998 \\
\hline C & -1.57544 & -5.67851 & 3.88140 \\
\hline $\mathrm{H}$ & -2.56128 & -5.43304 & 3.49788 \\
\hline C & -0.12588 & -7.06217 & 5.23079 \\
\hline $\mathrm{H}$ & 0.01123 & -7.90552 & 5.90131 \\
\hline C & 0.79763 & -5.20362 & 3.99327 \\
\hline $\mathrm{H}$ & 1.64788 & -4.60508 & 3.68781 \\
\hline C & -4.14441 & 6.60726 & -2.83603 \\
\hline $\mathrm{H}$ & -4.56126 & 7.55329 & -3.16919 \\
\hline C & -1.39831 & -6.75621 & 4.74599 \\
\hline $\mathrm{H}$ & -2.25399 & -7.35721 & 5.03914 \\
\hline B & -4.61748 & -1.91419 & 1.84953 \\
\hline $\mathrm{H}$ & -4.51906 & -2.05652 & 3.03616 \\
\hline C & -2.76561 & 6.40027 & -2.87609 \\
\hline $\mathrm{H}$ & -2.10557 & 7.18332 & -3.23740 \\
\hline C & 0.96950 & -6.28414 & 4.85523 \\
\hline $\mathrm{H}$ & 1.96118 & -6.51932 & 5.23006 \\
\hline B & -3.87374 & -0.49592 & 1.03950 \\
\hline
\end{tabular}




\begin{tabular}{|c|c|c|c|}
\hline $\mathrm{H}$ & -3.33046 & 0.17317 & 1.88221 \\
\hline$N$ & -0.98023 & -2.52189 & 2.86225 \\
\hline $\mathrm{N}$ & -2.44005 & 2.55991 & -0.17203 \\
\hline T & -2.17692 & 1.83911 & -2.18642 \\
\hline C & -2.53859 & 2.90361 & -1.45657 \\
\hline C & -0.71211 & -3.79340 & 2.54638 \\
\hline B & -3.65955 & -2.46324 & -0.62664 \\
\hline $\mathrm{H}$ & -2.89220 & -2.97621 & -1.39227 \\
\hline B & -4.71227 & -1.06082 & -1.34723 \\
\hline $\mathrm{H}$ & -4.79226 & -0.92472 & -2.54253 \\
\hline B & -4.53587 & -3.28991 & 0.71622 \\
\hline $\mathrm{H}$ & -4.51715 & -4.43568 & 1.06257 \\
\hline C & -3.30311 & -2.21533 & 0.89408 \\
\hline C & -3.81551 & -0.00153 & -0.46745 \\
\hline B & -5.30370 & 0.33141 & -0.27244 \\
\hline $\mathrm{H}$ & -5.91061 & 1.33048 & -0.59204 \\
\hline B & -5.73186 & -0.65889 & 1.25356 \\
\hline B & -6.26958 & -1.13886 & -0.35702 \\
\hline B & -6.04746 & -2.36588 & 0.88780 \\
\hline B & -5.36200 & -2.64776 & -0.75203 \\
\hline $\mathrm{H}$ & -6.39721 & -0.11355 & 2.09385 \\
\hline $\mathrm{H}$ & -7.38596 & -0.96927 & -0.78721 \\
\hline $\mathrm{H}$ & -6.99380 & -2.96231 & 1.34044 \\
\hline $\mathrm{H}$ & -5.84604 & -3.36772 & -1.59170 \\
\hline C & -0.69840 & -5.07847 & 0.33213 \\
\hline C & 3.90407 & -3.95991 & 0.18743 \\
\hline $\mathrm{N}$ & 3.48663 & -2.88352 & -0.73122 \\
\hline C & 5.41387 & -3.89473 & 0.44342 \\
\hline $\mathrm{H}$ & 5.70754 & -4.71058 & 1.11293 \\
\hline $\mathrm{H}$ & 5.96645 & -4.00070 & -0.49442 \\
\hline $\mathrm{H}$ & 5.69526 & -2.94630 & 0.90155 \\
\hline C & 3.14602 & -3.71384 & 1.49778 \\
\hline $\mathrm{H}$ & 2.06486 & -3.73234 & 1.33107 \\
\hline $\mathrm{H}$ & 3.41342 & -4.48741 & 2.22464 \\
\hline $\mathrm{H}$ & 3.39459 & -2.73708 & 1.92047 \\
\hline C & 3.56072 & -5.36007 & -0.34091 \\
\hline $\mathrm{H}$ & 3.68124 & -6.07597 & 0.47777 \\
\hline $\mathrm{H}$ & 2.53146 & -5.42288 & -0.69566 \\
\hline $\mathrm{H}$ & 4.22343 & -5.66760 & -1.15176 \\
\hline$N$ & -0.83558 & -3.91020 & 1.22208 \\
\hline C & 0.25984 & -6.14344 & 0.88391 \\
\hline $\mathrm{H}$ & 0.48916 & -6.85046 & 0.08072 \\
\hline $\mathrm{H}$ & 1.19863 & -5.71065 & 1.23029 \\
\hline $\mathrm{H}$ & -0.18309 & -6.70731 & 1.70659 \\
\hline C & -0.14560 & -4.53294 & -0.99033 \\
\hline $\mathrm{H}$ & -0.82097 & -3.78878 & -1.42024 \\
\hline $\mathrm{H}$ & 0.82030 & -4.04373 & -0.83421 \\
\hline $\mathrm{H}$ & -0.02669 & -5.35401 & -1.70442 \\
\hline C & -2.06374 & -5.73283 & 0.09243 \\
\hline $\mathrm{H}$ & -1.94489 & -6.59750 & -0.56974 \\
\hline $\mathrm{H}$ & -2.49341 & -6.07752 & 1.03733 \\
\hline
\end{tabular}




\section{References}

1. Y.-P. Zhou, S. Raoufmoghaddam, T. Szilvási, M. Driess, Angew. Chem. Int. Ed. 2016, 55, $12868-12872$.

2. R. S. Ghadwal, H. W. Roesky, S. Merkel, J. Henn, D. Stalke, Angew. Chem. Int. Ed. 2009, 48, 5683-5686.

3. G. M. Sheldrick, SHELX-97 Program for Crystal Structure Determination, Universität Göttingen (Germany) 1997.

4. Gaussian 09, Revision D.01, M. J. Frisch, G. W. Trucks, H. B. Schlegel, G. E. Scuseria, M. A. Robb, J. R. Cheeseman, G. Scalmani, V. Barone, G. A. Petersson, H. Nakatsuji, X. Li, M. Caricato, A. Marenich, J. Bloino, B. G. Janesko, R. Gomperts, B. Mennucci, H. P. Hratchian, J. V. Ortiz, A. F. Izmaylov, J. L. Sonnenberg, D. Williams-Young, F. Ding, F. Lipparini, F. Egidi, J. Goings, B. Peng, A. Petrone, T. Henderson, D. Ranasinghe, V. G. Zakrzewski, J. Gao, N. Rega, G. Zheng, W. Liang, M. Hada, M. Ehara, K. Toyota, R. Fukuda, J. Hasegawa, M. Ishida, T. Nakajima, Y. Honda, O. Kitao, H. Nakai, T. Vreven, K. Throssell, J. A. Montgomery, Jr., J. E. Peralta, F. Ogliaro, M. Bearpark, J. J. Heyd, E. Brothers, K. N. Kudin, V. N. Staroverov, T. Keith, R. Kobayashi, J. Normand, K. Raghavachari, A. Rendell, J. C. Burant, S. S. Iyengar, J. Tomasi, M. Cossi, J. M. Millam, M. Klene, C. Adamo, R. Cammi, J. W. Ochterski, R. L. Martin, K. Morokuma, O. Farkas, J. B. Foresman, and D. J. Fox, Gaussian, Inc., Wallingford CT, 2016.

5. A.D. Becke, J. Chem. Phys. 98, 5648 (1993).

6. (a) S.Grimme, S.Ehrlich, L.Goerigk, J Comput Chem, (2011), 32, 1456-1465; (b) S.Grimme, J.Antony, S.Ehrlich and H.Krieg, J.Chem.Phys., 132, (2010), 154104.

7. (a) R. Ditchfield, W. J. Hehre, and J. A. Pople, J. Chem. Phys., 54 (1971) 724; (b) W. J. Hehre, R. Ditchfield, and J. A. Pople, J. Chem. Phys., 56 (1972) 2257; (c) P. C. Hariharan and J. A. Pople, Theor. Chem. Acc., 28 (1973) 213-22; (d) P. C. Hariharan and J. A. Pople, Mol. Phys., 27 (1974) 209-14; (e) M. M. Francl, W. J. Pietro, W. J. Hehre, J. S. Binkley, D. J. DeFrees, J. A. Pople, and M. S. Gordon, J. Chem. Phys., 77 (1982) 3654-65; (f) J.-P. Blaudeau, M. P. McGrath, L. A. Curtiss, and L. Radom, J. Chem. Phys., 107 (1997) 5016-21; (g) A. J. H. Wachters, J. Chem. Phys., 52 (1970) 1033; (h) L. A. Curtiss, M. P. McGrath, J.-P. Blaudeau, N. E. Davis, R. C. Binning Jr., and L. Radom, J. Chem. Phys., 103 (1995) 6104-13.

8. NBO 6.0. E. D. Glendening, J. K. Badenhoop, A. E. Reed, J. E. Carpenter, J. A. Bohmann, C. M. Morales, C. R. Landis, and F. Weinhold (Theoretical Chemistry Institute, University of Wisconsin, Madison, WI, 2013); http://nbo6.chem.wisc.edu/ 